University of Tennessee Health Science Center

UTHSC Digital Commons

$10-2020$

\title{
Validation and Application of a Novel Target-Based Whole-Cell Screen to Identify Antifungal Compounds
}

Christian Alexander DeJarnette

University of Tennessee Health Science Center

Follow this and additional works at: https://dc.uthsc.edu/dissertations

Part of the Analytical, Diagnostic and Therapeutic Techniques and Equipment Commons, Bacterial Infections and Mycoses Commons, Chemicals and Drugs Commons, Medical Biochemistry Commons, Medical Genetics Commons, and the Pharmaceutics and Drug Design Commons

\section{Recommended Citation}

DeJarnette, Christian Alexander (https://orcid.org/0000-0002-9733-9152), "Validation and Application of a Novel Target-Based Whole-Cell Screen to Identify Antifungal Compounds" (2020). Theses and Dissertations (ETD). Paper 539. http://dx.doi.org/10.21007/etd.cghs.2020.0518. 


\title{
Validation and Application of a Novel Target-Based Whole-Cell Screen to Identify Antifungal Compounds
}

\begin{abstract}
Traditional approaches to drug discovery are inefficient and have several key limitations that constrain our capacity to rapidly identify and develop novel experimental therapeutics. To address this, we have devised a second-generation target-based whole-cell screening assay based on the principles of competitive fitness, which can rapidly identify target-specific and physiologically-active compounds. Briefly, strains expressing high, intermediate, and low levels of a preselected target protein were constructed, tagged with spectrally distinct fluorescent proteins (FPs), and mixed together. The pooled strains were then grown in the presence of various small molecules, and the relative growth of each strain within the mixed culture was compared by measuring the intensity of the corresponding FP tags. Chemically-induced population shifts indicated that the bioactivity of a small molecule was dependent upon the target protein's abundance and thus established a functional interaction. Here, we described the molecular tools required to apply this technique in the prevalent human fungal pathogen Candida albicans and validated this approach using dihydrofolate reductase. However, our approach, which we have termed target abundance-based fitness screening (TAFiS), should be applicable to an extensive array of molecular targets and in any genetically tractable microbe.

While the folate biosynthetic pathway has provided a rich source of antibacterial, antiprotozoal, and anticancer therapies, it has not yet been exploited to develop uniquely antifungal agents. Although there have been attempts to develop fungal-specific inhibitors of dihydrofolate reductase, the protein itself has not been unequivocally validated as essential for fungal growth or virulence. The purpose of this study was to establish dihydrofolate reductase as a valid antifungal target. Using a strain with doxycyclinerepressible transcription of DFR1 (PTETO-DFR1), we were able to demonstrate that Dfr1p is essential for growth in vitro. Furthermore, nutritional supplements of most forms of folate are not sufficient to restore growth when DFR1 expression is suppressed, or when its activity is directly inhibited by methotrexate, indicating that Candida albicans has a limited capacity to acquire or utilize exogenous sources of folate. Finally, the PTETO-DFR1 strain was rendered avirulent in a mouse model of disseminated candidiasis upon doxycycline treatment. Collectively, these results confirm the validity of targeting dihydrofolate reductase, and by inference other enzymes in the folate biosynthetic pathway as a strategy to devise new and efficacious therapies to combat life-threatening invasive fungal infections.

Both the fungal fatty acid synthase and desaturase have been established as essential for fungal growth, virulence in a systemic infection, and are structurally distinct from their mammalian counterparts. However, there has been little success in developing clinically relevant inhibitors that target the fungal fatty acid biosynthetic pathway, even though there are agents that inhibit the bacterial orthologs. The purpose of this study was to identify physiologically-active compounds that interact with the fungal fatty acid biosynthetic pathway. Given the challenging nature of targeting these enzymes using traditional approaches, we attempted to apply the newly validated TAFiS method, a target-based whole-cell screen. To accomplish this, Candida albicans strains with altered expression of the fungal fatty acid synthase or the desaturase were used to screen a total of 41,261 small molecules. The primary hits were confirmed through dose responses, and 16 compounds had differential activity on the strains with altered desaturase abundance, indicating a functional chemical-target interaction. Additionally, the growth inhibition exerted by the most potent hits was restored in the presence of exogenous fatty acids, further supporting their inhibition of the fatty acid desaturase. Of the 16 confirmed hits, 11 were structurally related, containing a hydrazide core that was essential for interaction with the desaturase. Collectively, these results have identified a novel scaffold that interacts with the fungal fatty acid desaturase and can be further developed into experimental therapeutics. It also confirms that the TAFiS methodology is highly
\end{abstract}


effective in identifying small molecules that functionally interact with targets that are poorly suited to traditional chemical screening approaches.

\section{Document Type}

Dissertation

Degree Name

Doctor of Philosophy (PhD)

\section{Program}

Biomedical Sciences

\section{Research Advisor}

Glen E. Palmer, PhD

\section{Keywords}

Candida albicans, Dihydrofolate Reductase, Drug discovery, Fungal Fatty acid synthase, Target Based Whole Cell Screening

\section{Subject Categories}

Analytical, Diagnostic and Therapeutic Techniques and Equipment | Bacterial Infections and Mycoses | Chemicals and Drugs | Diseases | Medical Biochemistry | Medical Genetics | Medical Sciences | Medicine and Health Sciences | Pharmaceutics and Drug Design | Pharmacy and Pharmaceutical Sciences 
DOCTOR OF PHILOSOPHY DISSERTATION

\section{Validation and Application of a Novel Target- Based Whole-Cell Screen to Identify Antifungal Compounds}

Author:

Christian Alexander DeJarnette
Advisor:

Glen E. Palmer, PhD

A Dissertation Presented for The Graduate Studies Council of

The University of Tennessee Health Science Center in Partial Fulfillment of the Requirements for the

Doctor of Philosophy degree from

The University of Tennessee

in

Biomedical Sciences: Microbiology, Immunology, and Biochemistry College of Graduate Health Sciences

October 2020 
Copyright $(92020$ by Christian Alexander DeJarnette.

All rights reserved. 


\section{DEDICATION}

To my Mother, Father, and Kaci. 


\section{ACKNOWLEDGEMENTS}

First, I would like to express sincere gratitude to my mentor. Glen Palmer. Thank you for all that you have taught me, encouraged me, and supported me.

Second, I would like to thank my committee members, Jeffery Becker, Jarrod Fortwendel, Dave Rogers, and Kirk Hevener. I appreciate each of you for your guidance and support.

Third, I would like to thank all of the members of the Palmer Lab, past and present for their friendship, encouragement, and support.

Finally, I am eternally grateful to all of my family and friends for their continued support in all of my endeavors.

The work described herein was supported by the Dean's Enhancement Program for Seed Research Grants (GEP) and National Institute of Health (NIH) grant R21 AI127607-01 (GEP). 


\begin{abstract}
Traditional approaches to drug discovery are inefficient and have several key limitations that constrain our capacity to rapidly identify and develop novel experimental therapeutics. To address this, we have devised a second-generation target-based wholecell screening assay based on the principles of competitive fitness, which can rapidly identify target-specific and physiologically-active compounds. Briefly, strains expressing high, intermediate, and low levels of a preselected target protein were constructed, tagged with spectrally distinct fluorescent proteins (FPs), and mixed together. The pooled strains were then grown in the presence of various small molecules, and the relative growth of each strain within the mixed culture was compared by measuring the intensity of the corresponding FP tags. Chemically-induced population shifts indicated that the bioactivity of a small molecule was dependent upon the target protein's abundance and thus established a functional interaction. Here, we described the molecular tools required to apply this technique in the prevalent human fungal pathogen Candida albicans and validated this approach using dihydrofolate reductase. However, our approach, which we have termed target abundance-based fitness screening (TAFiS), should be applicable to an extensive array of molecular targets and in any genetically tractable microbe.
\end{abstract}

While the folate biosynthetic pathway has provided a rich source of antibacterial, antiprotozoal, and anticancer therapies, it has not yet been exploited to develop uniquely antifungal agents. Although there have been attempts to develop fungal-specific inhibitors of dihydrofolate reductase, the protein itself has not been unequivocally validated as essential for fungal growth or virulence. The purpose of this study was to establish dihydrofolate reductase as a valid antifungal target. Using a strain with doxycycline-repressible transcription of DFRl (PTETO-DFRl), we were able to demonstrate that Dfr1p is essential for growth in vitro. Furthermore, nutritional supplements of most forms of folate are not sufficient to restore growth when DFRI expression is suppressed, or when its activity is directly inhibited by methotrexate, indicating that Candida albicans has a limited capacity to acquire or utilize exogenous sources of folate. Finally, the PTETO-DFRl strain was rendered avirulent in a mouse model of disseminated candidiasis upon doxycycline treatment. Collectively, these results confirm the validity of targeting dihydrofolate reductase, and by inference other enzymes in the folate biosynthetic pathway as a strategy to devise new and efficacious therapies to combat life-threatening invasive fungal infections.

Both the fungal fatty acid synthase and desaturase have been established as essential for fungal growth, virulence in a systemic infection, and are structurally distinct from their mammalian counterparts. However, there has been little success in developing clinically relevant inhibitors that target the fungal fatty acid biosynthetic pathway, even though there are agents that inhibit the bacterial orthologs. The purpose of this study was to identify physiologically-active compounds that interact with the fungal fatty acid biosynthetic pathway. Given the challenging nature of targeting these enzymes using traditional approaches, we attempted to apply the newly validated TAFiS method, a target-based whole-cell screen. To accomplish this, Candida albicans strains with altered 
expression of the fungal fatty acid synthase or the desaturase were used to screen a total of 41,261 small molecules. The primary hits were confirmed through dose responses, and 16 compounds had differential activity on the strains with altered desaturase abundance, indicating a functional chemical-target interaction. Additionally, the growth inhibition exerted by the most potent hits was restored in the presence of exogenous fatty acids, further supporting their inhibition of the fatty acid desaturase. Of the 16 confirmed hits, 11 were structurally related, containing a hydrazide core that was essential for interaction with the desaturase. Collectively, these results have identified a novel scaffold that interacts with the fungal fatty acid desaturase and can be further developed into experimental therapeutics. It also confirms that the TAFiS methodology is highly effective in identifying small molecules that functionally interact with targets that are poorly suited to traditional chemical screening approaches. 


\section{TABLE OF CONTENTS}

\section{CHAPTER 1. INVASIVE FUNGAL INFECTIONS AND THE NEED FOR

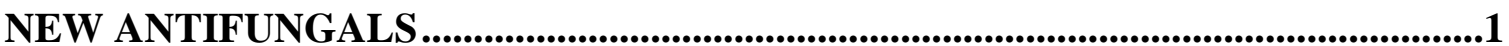

Fungal Infections Are a Significant Cause of Human Morbidity and Mortality ..............1

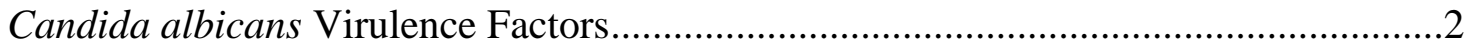

The Inadequacy of Current Antifungal Therapies ............................................................

Antifungal Drug Discovery: Traditional Approaches and Challenges .............................

Target-Based Whole-Cell Screens Greatly Improve the Efficiency of Drug

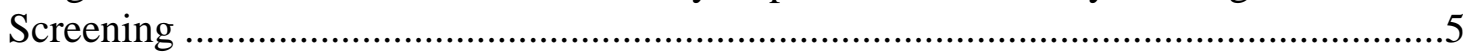

Hypothesis and Objective ………………………….............................................6

Validation of dihydrofolate reductase as an antifungal target .....................................6

Establish a novel target-based whole-cell screen.....................................................

Utilizing TAFiS to identify inhibitors of the fungal fatty acid biosynthetic

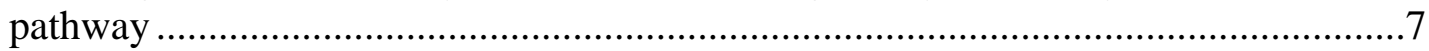

Structure-activity relationship of a known fatty acid desaturase inhibitor ...................8

\section{CHAPTER 2. DIHYDROFOLATE REDUCTASE IS A VALID TARGET FOR} ANTIFUNGAL DEVELOPMENT IN THE HUMAN PATHOGEN CANDIDA

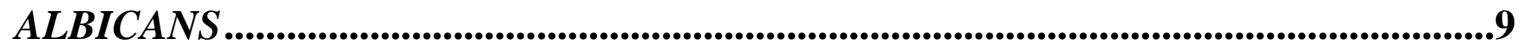

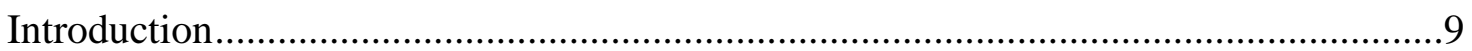

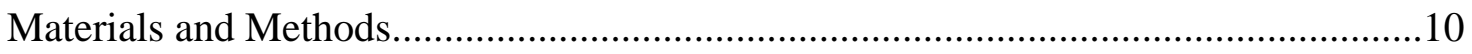

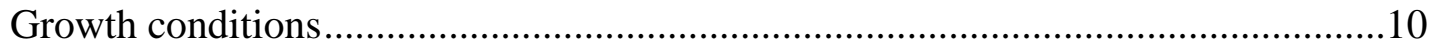

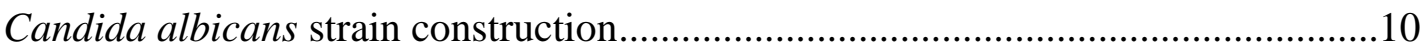

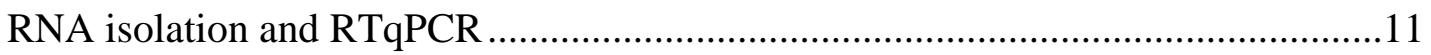

Antifungal susceptibility testing …………...........................................................11

Mouse model of disseminated candidiasis...............................................................13

Purification of the Candida albicans dihydrofolate reductase ..................................13

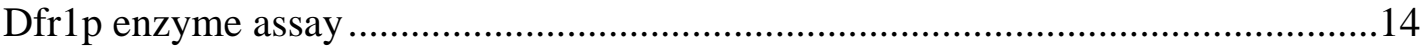

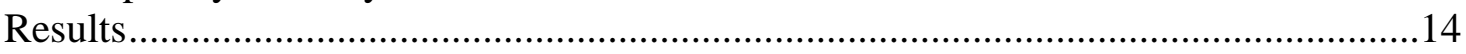

DHFR is essential for Candida albicans growth in vitro ..........................................14

Candida albicans has a limited capacity to utilize exogenous sources of folate .......16

DFRI is essential for Candida albicans virulence in a mouse model of disseminated infection ......................................................................................18

The pH-dependent antifungal activity of methotrexate is likely due to differential

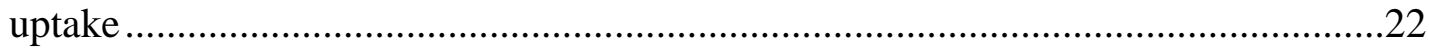

Established DHFR inhibitors are unable to inhibit Dfr1p within Candida

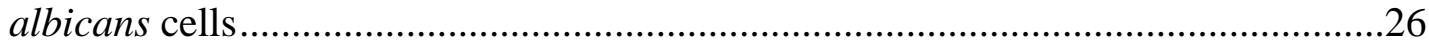

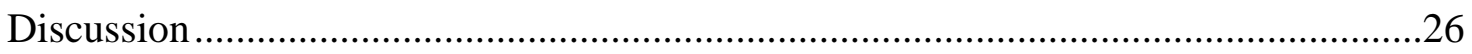

CHAPTER 3. TARGET ABUNDANCE-BASED FITNESS SCREENING (TAFIS) FACILITATES RAPID IDENTIFICATION OF TARGET-SPECIFIC AND PHYSIOLOGICALLY ACTIVE CHEMICAL PROBES ....................................31

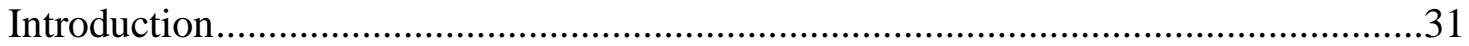

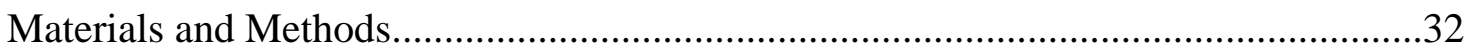




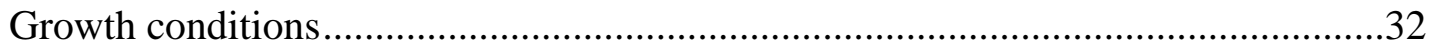

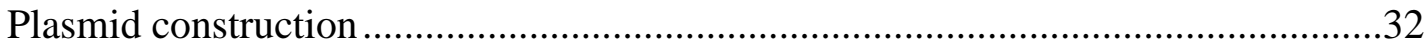

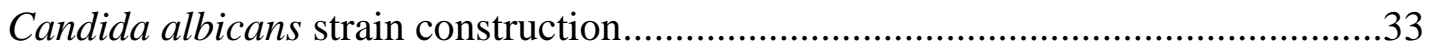

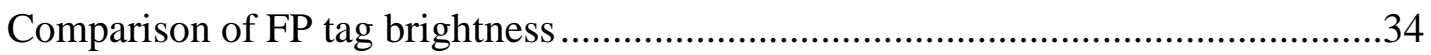

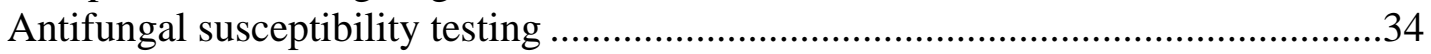

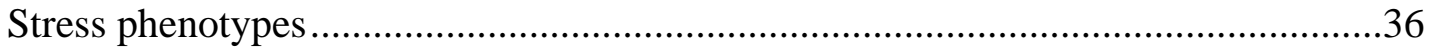

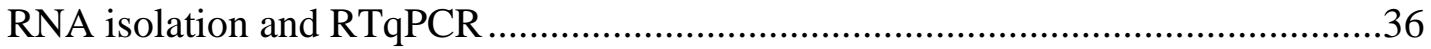

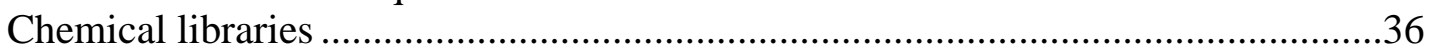

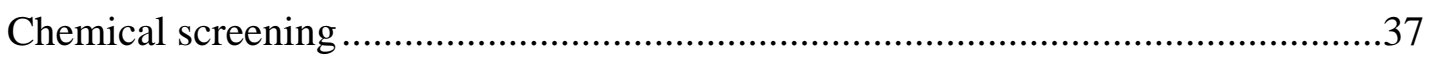

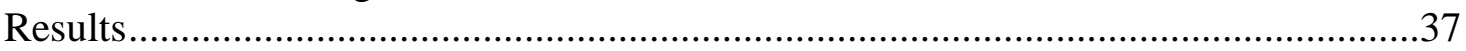

Design of target abundance-based fitness screening assay ........................................37

Selection and optimization of fluorescent protein tags...............................................37

Altering DFR1 expression dramatically affects Candida albicans methotrexate

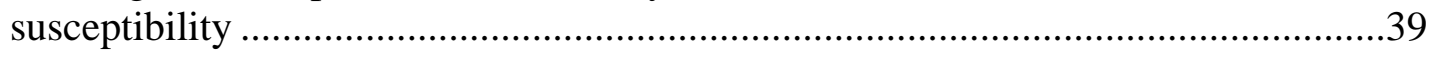

Chemical-target interactions can be detected as spectroscopic shifts in the FP-

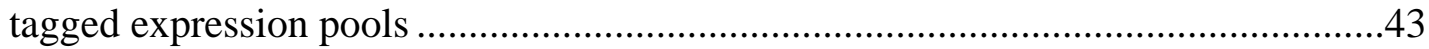

TAFiS reliably identifies on-target inhibitors.......................................................4

TAFiS can provide an effective and efficient high-throughput chemical screen .......44

TAFiS readily identified novel compounds as potentially interacting with Dfr $1 p$ by screening diverse chemical matter ......................................................................48

TAFiS is compatible with 384-well based high throughput screening formats..........52

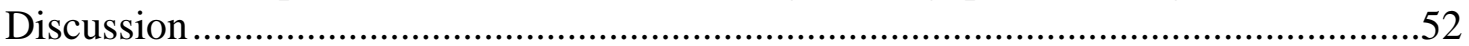

\section{CHAPTER 4. IDENTIFICATION AND STRUCTURE-ACTIVITY \\ RELATIONSHIPS OF INHIBITORS OF FUNGAL FATTY ACID}

BIOSYNTHESIS......................................................................................................................57

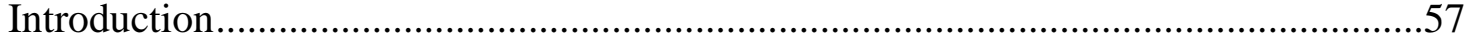

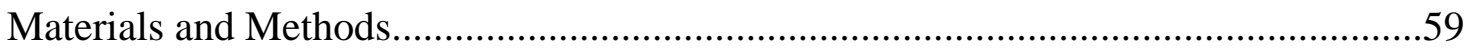

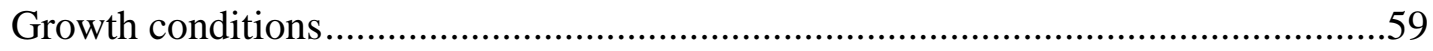

Plasmid construction ....................................................................................59

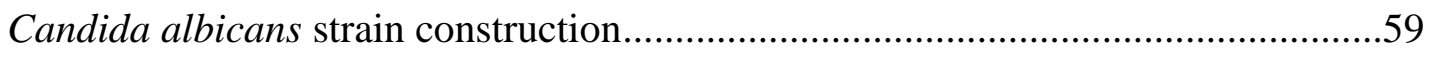

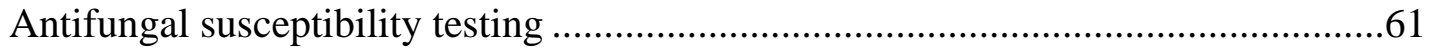

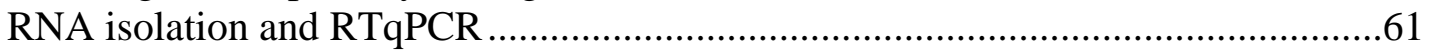

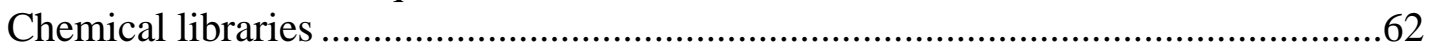

Chemical screening ............................................................................................62

Restoring growth in the presence of hit compounds by supplementation of fatty

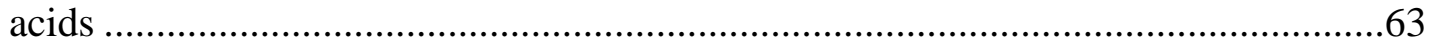

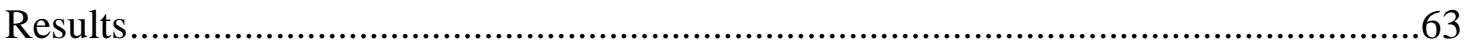

TAFiS provides high-throughput compatible screens to detect FAS and Ole1p

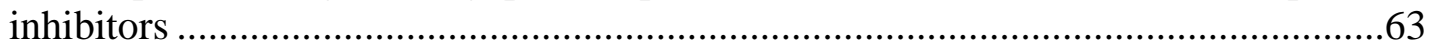

Identification of potential fatty acid biosynthesis inhibitors.......................................66

The supplementation with unsaturated fatty acids restores the growth of Candida albicans's growth in the presence of four hydrazide containing screening hits .........72 The Ole $1 \mathrm{p}$ inhibitors are structurally related and rely on a hydrazide moiety for activity 
The Ole1p inhibitor ECC190 contains a 1,2,4-triazolidine-3-thione core that degrades to produce acylhydrazones.............................................................. 77

The acylhydrazone derivatives of the 1,2,4-triazolidine scaffold act through the

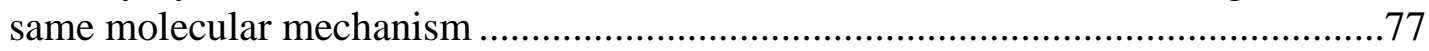

The presence and identity of the thiazole linker is important for potency of the 1,2,4-triazolidine scaffold

The size and aromaticity of the phenyl ring are important for the acylhydrazone to interact with Ole1p and inhibit the growth of Candida albicans

The acylhydrazone's ability to interact with Ole1p and inhibit Candida albicans growth requires hydrophobic functional groups attached directly to the hydrazone core

Discussion...

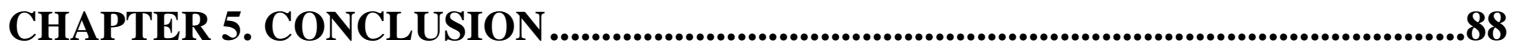

Introduction.

Chapter 2: Dihydrofolate Reductase Is a Valid Target for Antifungal Development

in the Human Pathogen Candida albicans .88

Chapter 3: Target Abundance-Based Fitness Screening (TAFiS) Facilitates Rapid Identification of Target-Specific and Physiologically Active Chemical Probes 90

Chapter 4: Identification of Inhibitors of Fungal Fatty Acid Biosynthesis

Conclusions. 


\section{LIST OF TABLES}

Table 2-1. Oligonucleotides used in Chapter 2...................................................12

Table 2-2. Antifolates inhibit the activity of Candida albicans dihydrofolate

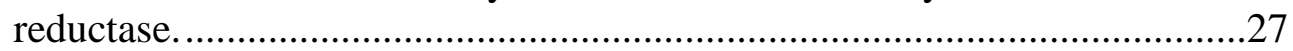

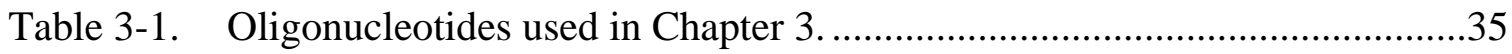

Table 3-2. Hit list from validation of TAFiS methodology using the first DFRI

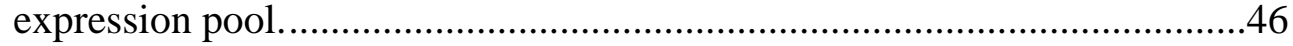

Table 3-3. Hit list from TAFiS validation using the second $D F R I$ expression pool. ....49

Table 3-4. Hit list from TAFiS validation using the second DFRl expression pool in the absence of folate and PABA. .............................................................50

Table 4-1. Oligonucleotides used in Chapter 4..................................................60

Table 4-2. Description of screening results.......................................................69

Table 4-3. Structures of compounds from the Myria collection identified as causing statistically significant population shifts in either FAS or Ole $1 p$ expression pools.

Table 4-4. Representative compounds from the structure-activity relationship testing of FAI354 and FAI409.

Table 4-5. Activity and potency of the analogs of ECC190 and FAI101. 


\section{LIST OF FIGURES}

Figure 2-1. Analysis of DFR1 transcript expression in Candida albicans PTETO-

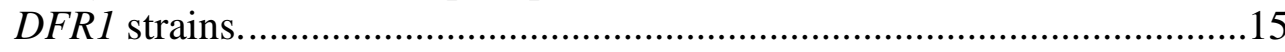

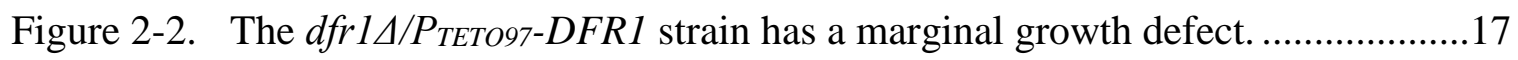

Figure 2-3. Dfr1p is essential for Candida albicans growth in vitro............................17

Figure 2-4. Excess folinic acid partially restores the growth Candida albicans in the presence of methotrexate.

Figure 2-5. Schematic representation of the folate biosynthetic pathway.

Figure 2-6. Folinic acid restores growth of Candida albicans in the presence of methotrexate.

Figure 2-7. Dfr1p is essential for Candida albicans virulence in a mouse model of disseminated candidiasis.

Figure 2-8. Methotrexate has pH-dependent antifungal activity on Candida albicans...23

Figure 2-9. The presence of exogenous folate does not alter Candida albicans sensitivity to methotrexate.

Figure 2-10. Dfr1p is essential for Candida albicans growth in both acidic and neutral conditions.

Figure 2-11. Efflux pump expression does not substantially alter Candida albicans susceptibility to methotrexate. .25

Figure 2-12. The $\mathrm{pH}$ dependent antifungal activity of methotrexate is species specific...27

Figure 3-1. Schematic of the Target Abundance-based Fitness Screening (TAFiS) assay.

Figure 3-2. The relative percentage of each Candida albicans strain within a population can be accurately quantified by four spectrally distinct fluorescent proteins.

Figure 3-3. High level expression of the selected fluorescent proteins does not result in differential susceptibility to a variety of stress.

Figure 3-4. Modulating DFRI expression affects Candida albicans susceptibility to methotrexate.

Figure 3-5. The first DFRl expression pool only identifies compounds that specifically interact with the target protein. 
Figure 3-6. The DFRI expression pool identified methotrexate as interacting with Dfrlp.

Figure 3-7. Compounds that induced statistically significant negative $\mathrm{Z}$ scores do not induce population shifts.

Figure 4-1. Construction of strains with altered FAS1 and OLE1 expression.

Figure 4-2. Modulating FAS1 and OLE1 expression levels in Candida albicans affects susceptibility to cerulenin and ECC190 respectively. .65

Figure 4-3. Off-target inhibitors do not cause population shifts that favor the high expression strain in the expression pools.

Figure 4-4. The primary hits from the Ole1p screens induce the expected population shift in the OLE1 expression pool.

Figure 4-5. The presence of unsaturated fatty acids restores the growth of Candida albicans in the presence of Ole1p inhibitors.

Figure 4-6. Ole1p inhibitors have $\mathrm{pH}-\mathrm{dependent} \mathrm{activity} \mathrm{on} \mathrm{Candida} \mathrm{albicans.}$ .74

Figure 4-7. Substitutions on the aromatic ring do not alter the on-target activity of Ole1p hits, but the hydrazine core is essential. . .76

Figure 4-8. ECC190 degrades into a hydrazone containing compound...........................78

Figure 4-9. Structure and analysis of ECC190 and FAI101.

Figure 4-10. Restoration of growth in the presence of inhibitors with the addition of unsaturated fatty acids. 


\section{CHAPTER 1. INVASIVE FUNGAL INFECTIONS AND THE NEED FOR NEW ANTIFUNGALS}

\section{Fungal Infections Are a Significant Cause of Human Morbidity and Mortality}

Superficial infections of the skin, hair, or nails are estimated to affect 1.7 billion individuals annually [1]. Millions more suffer from mucosal fungal infections of the oral and reproductive tracts [2-4]. Both superficial and mucosal diseases severely impact the individual's quality of life, however neither infection is typically life-threatening. In contrast, the dissemination of fungal pathogens into the bloodstream, or invasive fungal infections (IFIs), are estimated to affect 3 million people each year globally, with mortality rates surpassing 50\% [1], despite appropriate antifungal treatment. The majority of IFIs can be attributed to species of the Aspergillus, Pneumocystis, Candida, and Cryptococcus genera [1]. Aspergillus species are estimated to account for more than 200,000 IFIs per year, with mortality rates ranging from 30-95\% [1], primarily caused by Aspergillus fumigatus, a ubiquitous mold. Likewise, Pneumocystis species are responsible for over 400,000 IFIs annually, with mortality rates of 20-80\% [1], and the widespread atypical fungus Pneumocystis jirovecii is the leading cause of these IFIs. Another 1,000,000 IFIs annually are attributed to species of Cryptococcus, typically the ubiquitous yeast Cryptococcus neoformans, with mortality rates of 20-70\% [1]. Finally, another 400,000 IFIs per year are due to Candida species with mortality rates ranging from $46-75 \%$ [1].

Candida spp. are the second leading cause of catheter-associated urinary tract infections, the third leading cause of central line-associated bloodstream infections, the third-highest cause of bloodstream infections in the intensive care unit, and are the fourth leading cause of nosocomial bloodstream infections [5-8]. In the U.S. alone, 25,000 new cases of disseminated candidiasis are reported annually [9] with an estimated health care cost of up to $\$ 4$ billion a year [10], and increased hospital length of stay by 10-21 days [11]. C. albicans causes the majority of all bloodstream Candida infections globally [12$16]$ with mortality rates that range $29-72 \%[5,17,18]$, although other non-albicans Candida species are increasing in incidence [19, 20]. In addition to IFIs, the overgrowth of Candida species can cause superficial, and mucosal infections. Superficial Candida infections can affect otherwise healthy individuals and are typically in warm, humid folds of adjacent skin where the primary anatomical barrier has been damaged [21]. Mucosal infections can manifest as either oropharyngeal candidiasis (OPC) or vulvovaginal candidiasis (VVC). OPC is estimated to afflict nearly every HIV/AIDS patient [22], and is often associated with oral cancers [23, 24]. While OPC is not life-threatening, it does pose serious quality of life problems including nutrient acquisition in adults and failure to thrive in infants [22]. VVC is estimated to impact $70-75 \%$ of women in their childbearing years, regardless of immunocompetency, with up to $10 \%$ of women developing recurrent infections defined as more than four episodes a year [2, 4]. VVC, and especially recurrent vulvovaginal candidiasis, have substantial negative impacts on both occupational and social behaviors [25]. 
In a healthy individual, the first line of defense against microbial infections is the anatomical barriers composed of the skin and mucosa $[10,26]$. The skin provides a physical barricade against pathogens, while mucosal surfaces utilize acidic $\mathrm{pH}$, mucus, and antimicrobial secretions $[22,26]$ to prevent microbes from invading the body. The second barrier to pathogenic microbes is the innate immune system. The innate response includes the complement cascade, tissue-resident phagocytes, and antimicrobial peptides [26]. The immunological barrier can be compromised in disease states such as HIV/AIDS, or suppressed during antirejection or antineoplastic therapies leaving the individual at risk for infection [1].

Increases in the number of patients whose normal barriers, either anatomical or immunological, have been compromised is concerning as these are the most at risk for fungal infections. The anatomic barriers are often disrupted during invasive surgery, insertion of catheters, or injury $[10,12]$. These disruptions allow the colonization of and dissemination to sterile sites by fungi. The immune system is the second line of defense against fungal infections but can be compromised by disease or medical intervention. The on-going HIV and type-2 diabetes epidemics are major risk factors for mucosal fungal infections [12, 22, 26], while immunosuppression for solid organ transplant or antineoplastic therapy are major risk factors for invasive fungal infections (IFIs) [12, 14]. Additionally, the use of broad-spectrum antibiotics increases the risk of Candida infections as they wipe out the native microflora allowing overgrowth of the fungi [14, $15,21]$. Improvements in medical and supportive care have and continue to expand the population susceptible to fungal infections, further increasing the need for antifungal agents.

\section{Candida albicans Virulence Factors}

C. albicans is native to the human microflora and an opportunistic pathogen as it survives and causes disease in anatomically and environmentally distinct niches. $C$. albicans is estimated to asymptomatically colonize the gastrointestinal tracts of nearly $60 \%$ of the human population [27], the reproductive tract of roughly $70 \%$ of the population [2], and the oral mucosa of up to $80 \%$ [22]. This opportunistic pathogen's ability to occupy and infect such disparate environments is supported by a myriad of virulence factors. The expression of adhesins enables $C$. albicans to attach to biotic and abiotic surfaces, and are essential for both pathogenicity and biofilm formation [17, 28]. Once attached, C. albicans secretes hydrolytic enzymes, phospholipases and secreted aspartyl proteases, that both facilitate the active invasion of host tissues and extract nutrients from the extracellular environment [29]. C. albicans also secretes candidalysin, the first fungal cytolytic peptide toxin, which directly damages host cells [30]. However, C. albicans ability to reversibly switch morphology between the isotropic yeast form and the filamentous hyphal and pseudohyphal forms is thought to indicate the transition from a harmless commensal to a life-threatening pathogen [17]. In addition, morphological transitions allow the expression of hyphal specific genes that promote virulence, including adhesins and hydrolytic proteins, indicating that morphology switching is key to $C$. albicans pathogenicity. 


\section{The Inadequacy of Current Antifungal Therapies}

Current antifungal therapies are woefully inadequate to combat life-threatening infections as the unacceptably high mortality rates occur even with the appropriate use of the three antifungal drug classes [31-38]. Treatment of IFIs is severely limited by the significant restrictions of these therapies, including rising fungal resistance, patient toxicity, or a narrow spectrum of activity [31-38]. Azoles are the most widely used class of antifungals due to broad-spectrum activity and relatively low patient toxicity [39, 40]. This class inhibits lanosterol demethylase (Erg11p) in the ergosterol biosynthetic pathway, depleting ergosterol and producing toxic sterol intermediates that inhibit fungal growth [41]. Currently, only $0.5-2 \%$ of $C$. albicans bloodstream isolates are resistant to fluconazole, the most commonly used azole, and only $7 \%$ of all Candida bloodstream isolates are resistant [42]. However, resistance is rising, especially with the emergence of inherently resistant species such as Candida auris [36]. Despite these low resistance rates, between $19 \%$ and $46 \%$ of patients will not respond to treatment with the azoles, even when the fungal isolate is determined to be susceptible according to current MIC $_{50}$ breakpoints [43-48]. Worryingly, these treatment failures are not resistance-related, and therefore indicate the only modest efficacy of these drugs. The emerging resistance and clinical failures will soon limit the therapeutic value of this class.

A second antifungal class, the polyenes, contains only one drug that is approved for systemic use, amphotericin B. Amphotericin B directly interacts with ergosterol in the fungal membrane to induce stress and cell death. Previously, the drug was thought to intercalate into the membrane to form pores, which cause the loss of membrane integrity [49]. Recently, it was reported that the drug forms extramembrane aggregates that remove ergosterol, thus destabilizing the plasma membrane [50]. Regardless of the mechanism, amphotericin B has potent fungicidal activity with broad-spectrum activity, making this drug a robust last line of therapy for complicated IFIs [40]. Amphotericin B has a higher affinity for ergosterol than cholesterol driving fungal selectivity [40], but there is still substantial patient toxicity, including nephrotoxicity and renal failure, which were associated with substantial increases in morbidity and mortality [51]. Liposomal formulations of amphotericin B were developed that reduce the adverse effects [51, 52]. Fortunately, clinical resistance to amphotericin B is rare [53]. However, up to $38 \%$ of patients treated with amphotericin B will fail to respond to treatment $[54,55]$.

The third antifungal class, the echinocandins, are the most recently approved [56]. This class causes the loss of cell wall integrity by non-competitively inhibiting 1,3- $\beta$-Dglucan synthase (Fks1p), an enzyme that makes the essential cell wall component 1,3- $\beta$ D-glucan [57-59]. The absence of a cell wall in mammalian cells limits patient toxicity allowing the echinocandins to selectively target fungal cells. However, this class has a narrow spectrum of activity, and is only available as intravenous formulations, limiting their clinical utility [59]. Currently, resistance is low, but emerging as roughly $3 \%$ of Candida glabrata isolates are resistant to the echinocandins, in addition to their inherent resistance to fluconazole [42]. Furthermore, between $27 \%$ and $48 \%$ of patients treated with the echinocandin, caspofungin, fail therapy $[48,54,55,60,61]$. Therefore, it is clear 
that current antifungal agents are inadequate to combat IFIs, and we have an urgent need to develop new therapies that improve patient outcomes.

\section{Antifungal Drug Discovery: Traditional Approaches and Challenges}

The process of drug discovery, development, and approval is estimated to cost $\$ 2.5$ billion, and to take over a decade [62]. The preclinical phase is estimated at nearly $31 \%$ of the total cost and accounted for $50 \%$ of the timeline [62]. Thus, there is an urgent need to increase the efficiency of discovering and developing novel therapeutics. This need is especially crucial with antifungals, as the most recent drug class, the echinocandins, took thirty years to reach patients [56]. This lengthy timeline and expense is a direct result of the high attrition rate of promising drug candidates. Many failures are due to conventional screening assays that identify primary hits lacking essential aspects of effective therapies, including target specificity or membrane permeability. By identifying compounds that lack drug-like properties, conventional approaches require significant time and investment to identify the compounds that possess the appropriate characteristics. This inadequacy is a direct result of the limitations of the two most prevalent drug screening methodologies [63]. The first, target-based screens, require the development of a biochemical assay to detect modulators of a purified target protein's activity. While this approach often yields potent inhibitors of the purified protein, many of these hits lack activity on whole cells due to their insufficient membrane permeability or an inability to engage the molecular target in its native environment [64]. This necessitates costly efforts to improve cell permeability and/or selective target engagement that are often unsuccessful [65]. In addition, target-based screens are not applicable to many proteins, either because they are not amenable to purification or because the enzymatic assay is not high-throughput (HTP) compatible. Therefore many hits from target-based screens have not developed into clinical therapies [64], and a substantial fraction of time and resources have been lost to poor quality hits.

The alternative approach is whole-cell, or phenotypic, screening which identifies compounds that induce or correct disease-relevant phenotypes, such as inhibiting microbial growth. While these screens identify compounds that are active on whole cells, they do not provide information on the cellular target or the mechanism of action (MOA), both of which have to be identified in prolonged and costly subsequent studies [66]. Thus both of these screening strategies require substantial follow up in preclinical stages of development to triage the significant fraction of compounds that are viable. Therefore, the rapid identification of primary screening hits that exert physiological activity via a defined molecular target, coupled with the elimination of compounds that lack targetspecificity and whole-cell activity, should substantially increase the efficiency of this stage in drug discovery.

One of the major problems in antifungal drug discovery is that fungi are eukaryotic and, therefore, fundamentally similar to the human host. The similarity of the cellular signaling and biochemical pathways of the model fungus, Saccharomyces cerevisiae, indicates that these are highly conserved [67]. This conservation between 
fungi and humans dramatically reduces the number of available drug targets that can be successfully and safely exploited to derive antimicrobial molecules with fungal selective toxicity. Indeed, two of the three antifungal classes target ergosterol, a fungal specific sterol, and the third targets the cell wall, which is entirely absent in humans. In theory, fungal selective inhibitors are more likely to become clinical therapies, as human toxicity is a major reason for clinical trial failure $[68,69]$. Therefore conventional drug discovery's inefficiency and the fundamental similarity of fungal pathogens to the human host further hinders the development of selective antifungal agents that exploit novel targets.

\section{Target-Based Whole-Cell Screens Greatly Improve the Efficiency of Drug Screening}

A massive improvement in the efficiency of drug screening came from combining both of the conventional approaches in the form of $\underline{T}$ arget-ㅁased Whole-Cell $\underline{\text { Screens }}$ (TBWCS). These approaches rely on the principle of chemical genetics [70], where the abundance of the target protein determines the susceptibility to an on-target inhibitor. Therefore a strain with decreased target abundance would be hyper-susceptible to an ontarget inhibitor. Early TBWCS compared the growth between two strains, a reference strain and one that has decreased target protein abundance [71]. Primary screening hits were identified as those that affected the growth of the knockdown strain more than the growth of the reference strain. This differential growth inhibition indicates a functional interaction between the compound and the target of interest. Several studies with Staphylococcus aureus and Mycobacterium tuberculosis have collectively established the validity of TBWCS assays to discover on-target chemical probes [71-73].

TBWCS have many benefits over both of the traditional approaches. First, only compounds that interact with a predefined target of interest are identified, providing information on the target and MOA, from the primary screen. Second, only molecules that interact with the target in its native context, i.e. in intact cells, are selected, eliminating compounds with poor membrane permeability, those that fail to engage the intended target, or those that have substantial off-target effects. Third, by comparing the growth between two strains, this approach does not require the purification of the target or a high throughput (HTP) compatible biochemical assay for the primary screen. Therefore, this approach can be applied to targets that are traditionally challenging, such as non-catalytic proteins or those that are not amenable to purification. Thus, TBWCS have the potential to expedite the identification of a diverse physiologically active collection of agents that interact with a defined molecular target and eliminate compounds that have substantial off-target effects or are generally toxic.

Even with these advantages, TBWCS are underutilized and can be improved. Each of the previous efforts have been restricted by several technical issues that have limited efficiency, sensitivity, and/or throughput of the approach. First, previous TBWCS only utilized two strains to screen chemical libraries, a reference and a knockdown strain. The sensitivity of the assay is still limited as highly potent on-target compounds can overwhelm the dynamic range of the assay and have the same effect on both strains. 
Second, previous screens used two plate assays that compare the growth of reference and knockdown strains on separate culture plates, limiting the efficiency and throughput of the assay. Finally, most of the previous TBWCS relied on a semi-quantitative output, such as zones of growth inhibition, which are not designed for quantitative measurements, automated data collection, or statistical analysis [71, 74].

\section{Hypothesis and Objective}

The overall goal of this work is to validate a new methodology that will improve the efficiency, sensitivity, and throughput of drug screening as well as lay the groundwork to exploit three new drug targets for antifungal development. We hypothesize that our TAFiS methodology will increase the efficiency and throughput of TBWCS, and identify chemical modulators for conventionally challenging targets. The advantages of the TAFiS assay will save both time and money in the early stages of drug discovery, providing faster and more cost-effective novel antifungal therapies. To test this hypothesis we will apply and validate this methodology using a target from the wellcharacterized, and historically important folate pathway, but has not been validated in fungi. We will also apply our methodology to less characterized, more challenging targets in the fungal fatty acid synthesis pathway.

\section{Validation of dihydrofolate reductase as an antifungal target}

The folate (FOL) biosynthetic pathway has been successfully targeted in the development of antineoplastic, antibacterial, and antiprotozoal drugs. In this pathway, GTP is converted to dihydroneopterin triphosphate by GTP cyclohydolase I (Fol2p), and then subsequently condensed with PABA to form dihydropteroate by the subsequent actions of dihydroneopterin aldolase (DHNA), hydroxymethyldihydropterin pyrophosphokinase (HPPK) and dihydropteroate synthase (DHPS). In yeast the DHNA, HPPK, and DHPS are provided on a single trifunctional protein (Fol1p). Then dihydrofolate synthase (DHFS, Fol3p) adds a single L-glutamate residue to produce dihydrofolate, which is then reduced by dihydrofolate reductase (DHFR) to tetrahydrofolate. Notably, humans lack four of the enzymes required to produce folate, DHNA, HPPK, DHPS, and DHFS. Antineoplastics include FOL analogs such as methotrexate (MTX), a potent nonselective inhibitor of DHFR [75, 76]. Other notable antifolates include trimethoprim, a bacterial DHFR inhibitor, and a collection of 'sulfa drugs' including sulfamethoxazole that inhibit DHPS from bacterial and protozoan species [77]. DHFR inhibitors pyrimethamine, proguanil, chlorproguanil have been crucial to treat malaria [78-80], as well as the DHPS inhibitor dapsone [81]. A combination of trimethoprim and sulfamethoxazole can also provide an effective treatment for Pneumocystis pneumonia caused by the atypical fungus Pneumocystis jirovecii [82]. Although these drugs were not specifically developed to target the fungal folate biosynthetic enzymes of Pneumocystis, this provides clinical evidence that inhibition of the FOL pathway can provide an effective intervention strategy to treat invasive mycoses. However, conventional antifolate drugs that were developed for 
bacterial, protozoan, human FOL enzymes have little or no activity upon the major human fungal pathogens [83-85]. This lack of activity is either because of divergence of the fungal enzyme structures, or the inability of antifolates to enter fungal cells [83].

Furthermore, no published study has unequivocally validated the core components of the FOL pathway as essential for infectious fungi to cause disease within a mammalian host, and are therefore genuine and potentially efficacious targets for antifungal development. We hypothesize that the fungal DHFR is required by $C$. albicans to survive in vitro, and cause disease in vivo.

\section{Establish a novel target-based whole-cell screen}

Traditional drug screening approaches are inefficient and have several key limitations that hinder our capacity to identify and develop novel therapeutics. To address this, we have designed a second-generation TBWCS, called Target Abundance-based Fitness Screening (TAFiS) that incorporates the principle of competitive fitness, and should rapidly identify target-specific and physiologically active agents. Our approach increases the number of strains with altered target abundance compared to previous TBWCS, which produces more data points per compound, and increases the dynamic range of the assay by including strains with enhanced stratification of the target protein abundance. Additionally, all strains will be combined into a single population for screening, forcing the strains to compete with each other in the presence of screening compounds. By incorporating the principle of competitive fitness, the sensitivity of the assay should increase, as even minute fitness defects caused by the screening compound will become apparent in the competition between strains. Combining the strains together will also allow the screen to be conducted in a single plate, increasing the efficiency when compared to the two-plate assays previously employed. Finally, our approach enables the quantification of each strain's growth using fluorescence to facilitate rapid measurement and statistical analysis. This methodology could be universally applied to any potential drug-target in any genetically tractable microbe. We hypothesize that this methodology will increase the sensitivity and efficiency of drug screening by rapidly identifying primary screening hits with activity on intact cells that act through a defined molecular target and eliminate compounds that lack the desired characteristics of promising antifungal lead compounds. The purpose of this study was to validate the TAFiS methodology as a novel TBWCS approach to drug screening by generating the fluorescent protein constructs, the tools to alter the target abundance, and to ensure that screen identified known on-target inhibitors using lanosterol demethylase, target of the azoles, and DHFR.

\section{Utilizing TAFiS to identify inhibitors of the fungal fatty acid biosynthetic pathway}

Next, we applied our new TBWCS approach to identify inhibitors of fungal fatty acid synthesis. Fatty acids are vital constituents of membrane lipids, an important means to store metabolic energy, and are required for post-translational modifications of many essential proteins. There have been very few efforts to exploit this pathway in infectious 
fungi, even though the same biological processes have been successfully targeted in bacteria $[86,87]$. The proteins in this pathway are conventionally difficult drug-targets as they are not amenable to purification and do not have HTP compatible biochemical assay. We hypothesize that the TAFiS methodology could identify novel inhibitors of two traditionally challenging targets: the fungal fatty acid desaturase (Ole1p) and the fungal fatty acid synthase (FAS). We propose to develop strains that have an altered abundance of either FAS or Ole1p and then to identify novel inhibitors by screening chemical libraries using the TAFiS methodology.

\section{Structure-activity relationship of a known fatty acid desaturase inhibitor}

Finally, we determined the structure-activity relationship of a previously identified family of molecules with a 1,2,4-triazolidine-3-thione core that interacts with Ole1p [88]. Upon investigation, we noticed that one of the molecules had the potential to degrade into a remarkably similar hydrazone molecule to a previously published series of molecules with potent antifungal activity but no known target $[89,90]$. We synthesized the original molecule and found that the 1,2,4-triazolidine-3-thione molecule degraded into the predicted hydrazone product. This observation led us to investigate the aspects of the molecule's structure that determined the activity. We hypothesize that identifying the structural elements that drive this Ole1p inhibitor might help the development of future inhibitors and improvements upon this scaffold. 


\section{CHAPTER 2. DIHYDROFOLATE REDUCTASE IS A VALID TARGET FOR ANTIFUNGAL DEVELOPMENT IN THE HUMAN PATHOGEN CANDIDA ALBICANS ${ }^{I}$}

\section{Introduction}

The CDC estimates that the direct healthcare costs of treating fungal infections to be over $\$ 7$ billion each year in the U.S. alone [91]. Along with this high financial burden, over 1 million deaths are attributed to invasive fungal infections (IFIs) annually [1], despite the appropriate use of the available antifungal drugs. The most widely used antifungal drug class, the azoles, inhibit ergosterol biosynthesis, but the incidence of resistance is on the rise and starting to compromise their clinical utility [42]. The polyene amphotericin B disrupts fungal membranes through direct interaction with ergosterol [92]. However, despite broad-spectrum antifungal activity, this drug is associated with severe patient toxicity that limits its use. The most recently approved antifungal drugs are the echinocandins, which target cell wall biosynthesis. Drugs in this class are principally active against Candida species, and therefore have a narrow spectrum of activity and are only available in intravenous formulations, further limiting their clinical utility [38]. A larger concern is the modest efficacy of all three classes of antifungal drug, as this is likely a major determinant of the excessively high mortality rates in patients with IFIs, as well as the high rates of recurrent mucosal infections. For example, approximately a third of patients with disseminated Candida infections are non-responsive to treatment with fluconazole [43, 44, 46, 93, 94], voriconazole [47], or the recently approved isavuconazole [48], even among those infected by isolates deemed susceptible according to current clinical breakpoints. Favorable response rates of just 52 to $73 \%$ have been reported for patients with disseminated candidiasis when treated with an echinocandin $[48,54,55,60,61]$ and $62 \%$ with amphotericin B [54, 55]. Of grave concern is the recent isolation of $C$. auris from patients across the globe that is resistant to all three classes of antifungal drugs [36]. As such, there is an urgent need for new antifungal drugs with improved therapeutic efficacy, patient safety, and spectrum of activity.

In its reduced form, tetrahydrofolate (THF), folate is an essential coenzyme for a number of cellular enzymes, serving as a carrier for the transfer of one-carbon units as well as their interconversion between various oxidation states [95]. THF is required for the synthesis of thymidine monophosphate, purines, methionine, as well as a multitude of other important metabolites. Mammals are unable to synthesize folate as they lack several of the necessary enzymes and must acquire it through dietary intake. A specialized transport system is required for cellular uptake of folic acid, which is then converted into its active form, THF, by dihydrofolate reductase (DHFR) [96]. In contrast, the majority of prokaryotes and microbial eukaryotes lack the transport systems found in mammals and must therefore synthesize folic acid de novo.

\footnotetext{
${ }^{1}$ Reprinted from final submission with open access permission. DeJarnette C, Luna-Tapia A, Estredge LR, Palmer GE. 2020. Dihydrofolate reductase is a valid target for antifungal development in the human pathogen Candida albicans. mSphere 5:e00374-20. https://doi.org/10.1128/mSphere.00374-20. [97]
} 
The folate biosynthetic pathway has been targeted with enormous success in the development of antineoplastic, antibacterial, as well as antiprotozoal drugs. Anticancer drugs include folate analogs such as methotrexate (MTX), a potent inhibitor of DHFR $[75,76]$. Other notable antifolates include trimethoprim, which inhibits bacterial DHFR, and a collection of sulfa drugs including sulfamethoxazole that inhibit dihydropteroate synthase (DHPS) from some bacterial as well as protozoan parasites [77]. Antiprotozoal drugs that target this pathway have been especially important for the treatment of malaria and include the DHFR inhibitors pyrimethamine, proguanil, chlorproguanil [78-80], as well as the DHPS inhibitor dapsone [81]. Curiously, a combination of trimethoprim and sulfamethoxazole can also provide an effective treatment for Pneumocystis pneumonia caused by the atypical fungus Pneumocystis jirovecii [82]. Although these drugs were not specifically developed to target the folate biosynthetic enzymes of Pneumocystis, this provides clinical evidence that targeted inhibition of the folate (FOL) pathway can provide an effective intervention strategy to treat invasive mycoses. However, the conventional antifolate drugs developed for bacterial or protozoan DHPS or DHFR, or even to human DHFR, have little or no antifungal activity upon the major human fungal pathogens [83-85], either because of divergence of the fungal enzyme structures or permeability issues that prevent antifolates from entering fungal cells [83]. Furthermore, until now, no published study has unequivocally determined if the core components of the FOL pathway are required by infectious fungi to cause disease within a mammalian host, and are therefore valid and potentially efficacious targets for antifungal development. The objective of this study was to determine if DHFR is essential for $C$. albicans, one of the most prevalent human fungal pathogens, to survive and cause disease within its mammalian host.

\section{Materials and Methods}

\section{Growth conditions}

C. albicans was routinely grown on yeast extract-peptone-dextrose (YPD) agar plates at $30^{\circ} \mathrm{C}$, supplemented with $50 \mu \mathrm{g} / \mathrm{ml}$ uridine for ura3 $\Delta / \Delta$ strains. Selection of $C$. albicans transformants was carried out on minimal YNB medium $(6.75 \mathrm{~g} /$ liter yeast nitrogen base without amino acids, $2 \%$ dextrose, $2 \%$ Bacto agar) supplemented with the appropriate auxotrophic requirements described for $S$. cerevisiae [98] or with $50 \mu \mathrm{g} / \mathrm{ml}$ uridine.

\section{Candida albicans strain construction}

BWP17 [99] was kindly provided by Aaron Mitchell (Carnegie Mellon University). C. albicans was transformed with DNA constructs using the lithium acetate procedure [100]. The parental strain, BACTR31, was constructed by transforming BWP17 with pDUP3 [101] that contains the tetracycline repressor protein (TetR) from Nakayama and colleagues [102]. To generate this plasmid, TetR from THE1 [102] was 
PCR amplified using primers TetRHap4ADF-SalI and TetRHap4ADR-MluI, and then cloned into pDUP3 between $\mathrm{Sal}$ I and Mlu I sites. This pDUP3:TetR plasmid was linearized using Nae I and transformed into BWP17.

The $D F R 1$ gene deletion cassette was amplified using primer set DFR1DISF and DFR1DISR and plasmid pRSARG4 $\triangle$ SpeI ( $A R G 4$ selection marker) as a template and transformed into BACTR31. Correct integration of the gene deletion cassette to replace one DFRI allele was confirmed by diagnostic PCR, using primers ARG4INTR2 and DFR1AMPR-SacI as well as ARG4INTF2 and DFR1AMPF-KpnI. To make the $P_{\text {TETO- }}$ DFRl strain, we generated promoter replacement cassettes by PCR amplifying the tetracycline-repressible promoters from the plasmids p97CAU, p98CAU, or p99CAU [101] using the primers DFR1TETF and DFR1TETR. Each cassette was transformed into the BACTR31 derived DFR1/dfr1A:ARG4 heterozygote, and the presence of the PTETO was determined by diagnostic PCR using TETOSEQF and DFR1DETR. The absence of a wild-type allele was determined by diagnostic PCR using DFR1AMPF-KpnI and DFR1DETR. The strains were then made prototrophic by transforming pGEMHIS1 [103] linearized with Nru I. Oligonucleotides used in this study are listed in Table 2-1.

\section{RNA isolation and RTqPCR}

RNA was isolated using the hot phenol method of RNA isolation described previously [104]. This RNA pellet was washed with $500 \mu \mathrm{l}$ of $70 \%$ ice-cold ethanol and collected by centrifugation. The RNA pellet was resuspended in Dnase/Rnase-free $\mathrm{H}_{2} \mathrm{O}$. cDNA was synthesized from total RNA using the Verso cDNA Synthesis Kit (Thermo Scientific) in accordance with the manufacturer's instructions. Synthesized cDNA was used for both the amplification of ACTl and the gene of interest by PCR, using SYBR green PCR master mix, according to the manufacturer's instructions. Genespecific primers were designed using the PrimerQuest Tool from Integrated DNA Technologies (IDT). The PCR conditions consisted of an initial denaturation at $95^{\circ} \mathrm{C}$ for 10 minutes, followed by 40 cycles of denaturation $\left(95^{\circ} \mathrm{C}\right.$ for 15 seconds), annealing $\left(60^{\circ} \mathrm{C}\right.$ for 30 seconds), and extension $\left(72^{\circ} \mathrm{C}\right.$ for 37 seconds). Software for the 7500 System Sequence Detection Software version 1.2.3 from Applied Biosystems was used to determine the dissociation curve and threshold cycle (CT). The $2^{-\Delta \Delta} \mathrm{CT}$ method was used to calculate changes in gene expression among the strains [105]. All experiments included both biological and technical replicates in triplicate.

\section{Antifungal susceptibility testing}

Stock solutions of MTX were prepared at $10 \mathrm{mM}$ in DMSO and diluted as needed in the same solvent. Each C. albicans strain was grown overnight in YPD at $30^{\circ} \mathrm{C}$ and diluted to approximately 1 X $10^{4}$ cells $/ \mathrm{ml}$ in YNB or RPMI 1640 medium, and $100 \mu 1$ of each cell suspension was transferred to the wells of a round-bottom 96-well plate. An additional $100 \mu \mathrm{l}$ of YNB or RPMI 1640 medium containing $2 \mathrm{x}$ the final desired 
Table 2-1. Oligonucleotides used in Chapter 2.

\begin{tabular}{|c|c|}
\hline Primer & Sequence $5^{\prime} \rightarrow 3^{\prime}$ \\
\hline DFR1DISF & $\begin{array}{l}\text { TTCTTGTTTGATTGAAAAAAAACTTTCACCACTAATCTAGAACTT } \\
\text { CACGAATAGACAACAGTTTTCCCAGTCACGACGTT }\end{array}$ \\
\hline DFR1DISR & $\begin{array}{l}\text { TTATTTTCTTGTCCATAGCGTATAATTATAGGTAAAATCACCTTC } \\
\text { CTTGATATCGTCTTTGTGGAATTGTGAGCGGATA }\end{array}$ \\
\hline DFR1TETOF & $\begin{array}{l}\text { TGGACTTAGGGGTACTTGGCCAGGTATTGTATTTTTATCAGGTTA } \\
\text { TTCATTGTTGCACTCTAGGGTAATACGACTCACTATAGGG }\end{array}$ \\
\hline DFR1TETOR & $\begin{array}{l}\text { CTTTGTATCCAATTCCCAAAGCAGGCTTTAATGCCGCGACAATGA } \\
\text { TTGCAACATTTGGTTTCAGCATCTAGTTTTCTGAGATAAAGCTG }\end{array}$ \\
\hline ARG4INTF2 & AAGCTAGTGTGGAAAGAAGAG \\
\hline ARG4INTR2 & AATGACTGAATTATGTCGGTC \\
\hline $\begin{array}{l}\text { DFR1AMPF- } \\
\text { KpnI }\end{array}$ & TCAGGTACCTTGAGTTGTGGACTTAGTGGG \\
\hline $\begin{array}{l}\text { DFR1AMPR- } \\
\text { SacI }\end{array}$ & TCAGAGCTCCGACTTTGGAGAGAGAACAGC \\
\hline TETODETF & GTTGACACTTGTAAATAAGCG \\
\hline DFR1DETR & TTGGTCCAACTTTCCAATGGG \\
\hline $\begin{array}{l}\text { TetRHap4ADF- } \\
\text { Sal1 }\end{array}$ & TCAGTCGACATGTCTAGATTAGATAAAAGTAAAG \\
\hline $\begin{array}{l}\text { TetRHap4ADR } \\
\text { - Mlu1 }\end{array}$ & TCAACGCGTCAACTCAATTGAGGTTACTCGG \\
\hline DFR1q1 & TGTTGCAATCATTGTCGCGG \\
\hline DFR1q2 & CCTTACGGAGTCTCCAAGGC \\
\hline ACT1q1 & TTGGATTCTGGTGATGGTGTTA \\
\hline ACT1q2 & TCAAGTCTCTACCAGCCAAATC \\
\hline
\end{tabular}

Engineered restriction enzyme sites are underlined. 
concentration of MTX was then added to each well. The final concentration of DMSO was $0.5 \%$ for all treatments. Plates were then incubated at $30^{\circ} \mathrm{C}$ before growth was quantified after 24 and 48 hours by measuring $\mathrm{OD}_{600 \mathrm{~nm}}$ using a Cytation 5 plate reader (Bio-Tek Instruments, Inc.). MIC50 values were identified visually using the CLSI antifungal susceptibility assay and then confirmed by quantifying growth by $\mathrm{OD}_{600 \mathrm{~nm}}$. The growth of each strain at each drug concentration was then expressed relative to the DMSO-alone control. The susceptibility to MTX at the highest concentration in each $\mathrm{pH}$ was compared to the other $\mathrm{pH}$ using a two-tailed student's $\mathrm{t}$ test $\left({ }^{*} \mathrm{P}<0.01\right.$; ${ }^{* *} \mathrm{P}<$ $0.0001)$.

\section{Mouse model of disseminated candidiasis}

All animal experiments were done according to protocols approved by the University of Tennessee Institutional Animal Care and Use Committee. Groups of 6 to 8week-old female BALB/c mice (Charles River Laboratories) were randomly assigned to one of three treatment groups three days before infection. Group 1 (minus doxycycline) was provided $20 \mathrm{~g}$ of DietGel $\left(\right.$ Clear $\mathrm{H}_{2} \mathrm{O}$ ) per day, per cage of 4 mice, while group 2 (plus doxycycline) was provided the same diet supplemented with $2 \mathrm{mg} / \mathrm{ml}$ of doxycycline hyclate. Group 3 (post-treatment) were given the DietGel without doxycycline until 24 hours after infection and were administered $10 \mathrm{mg} / \mathrm{kg}$ of doxycycline through oral gavage at 12,24 , and 36 hours post-infection. Treatment was maintained daily for the duration of the experiment (up to 12 days post-infection). Each C. albicans strain was grown overnight in YPD cultures at $30^{\circ} \mathrm{C}(200 \mathrm{rpm})$. Cells were washed twice in sterile endotoxin-free phosphate-buffered saline (PBS), and cell density determined using a hemocytometer. Each strain was then diluted to 5 X $10^{6}$ cells $/ \mathrm{ml}$ in sterile PBS, and $0.1 \mathrm{ml}$ of each cell suspension inoculated into the lateral tail vein of mice from each treatment group. Viable cell counts of each inoculum were confirmed by plating appropriate dilutions onto YPD agar plates and counting the number of colonies formed after 48 hour. Mice were monitored for 12 days, and those showing distress euthanized. Survival data were plotted on a Kaplan-Meier curve and analyzed by Logrank (Mantel-Cox) test using GraphPad Prism 8.00. The kidneys from each mouse were extracted, weighed, and homogenized in PBS. Serial dilutions of kidney homogenate were plated on YPD agar plates containing $50 \mu \mathrm{g} / \mathrm{ml}$ of chloramphenicol. The number of CFU/gram of kidney tissue was determined from the number of colonies on the plates after 48 hours.

\section{Purification of the Candida albicans dihydrofolate reductase}

The $C$. albicans DFRI ORF sequence was retrieved from the Candida Genome Database [106], and was codon optimized for expression in E. coli. The optimized DFRI ORF was then cloned into pET15b [107] to allow propagation and expression of $C$. albicans Dfr1p in E. coli. Cultures of E. coli transformed with pET15b: CaDfr 1p (Biomatik) were grown overnight in LB plus ampicillin. The culture was diluted 1:100 into fresh media and incubated at $35^{\circ} \mathrm{C}$ for 6 hours, then Dfr $1 p$ expression was induced 
with $0.5 \mathrm{mM}$ IPTG final concentration for 16 hours. Cells were collected by centrifugation and resuspended in binding buffer $(6 \mathrm{~g} / 1$ Tris base $\mathrm{pH} 7.5,8.8 \mathrm{~g} / \mathrm{l} \mathrm{NaCl}, 1$ $\mathrm{mM}$ DTT, $0.68 \mathrm{~g} / \mathrm{l}$ imidazole, $5 \%$ glycerol, $1 \mathrm{X}$ protease inhibitor). Resuspended cells were lysed with $1 \mathrm{mg} / \mathrm{ml}$ chicken white lysozyme (Sigma) for $60 \mathrm{~min}$ at $4^{\circ} \mathrm{C}$. Then Triton $\mathrm{X} 100$ and $\mathrm{NaCl}$ were added to $0.1 \%$ and $0.5 \mathrm{M}$ respectively. The lysate was then centrifuged and the supernatant was collected. The supernatant was added to Nickel resin beads (Sigma) and incubated at $4^{\circ} \mathrm{C}$ for 30 min with rotation. The buffer was removed and the beads were washed twice with binding buffer. Then the beads were suspended in elution buffer (6 g/l Tris base $\mathrm{pH}$ 7.5, $8.8 \mathrm{~g} / \mathrm{l} \mathrm{NaCl}, 1 \mathrm{mM}$ DTT, $34 \mathrm{~g} / \mathrm{l}$ imidazole, 5\% glycerol), and incubated at $4^{\circ} \mathrm{C}$ for $30 \mathrm{~min}$. The elution was removed and then run on SDS-PAGE gels (Sigma) to confirm the presence of the CaDfr1p. SDS-PAGE gels were run according to the manufacturer's protocol.

\section{Dfr1p enzyme assay}

The enzymatic activity of $C$. albicans Dfrlp was determined using the dihydrofolate reductase assay (Sigma). The manufacturer protocol was adapted to the fungal enzyme by scaling down the volume to $100 \mu$ reactions with $88 \mu$ l buffer, $6 \mu \mathrm{l}$ of a $1 \mathrm{mM}$ NADPH stock, $5 \mu \mathrm{l}$ of a $1 \mathrm{mM}$ DHF stock, and $1 \mu \mathrm{l}$ of $3 \mathrm{mg} / \mathrm{ml}$ purified enzyme. The loss of NADPH was quantified as the decrease in absorption at OD $340 \mathrm{~nm}$ over a 30minute timeframe. The $\mathrm{IC}_{50}$ was calculated by converting the change in $\mathrm{OD}_{340 \mathrm{~nm}}$ per minute into THF produced per minute and then compared to an untreated control.

\section{Results}

\section{DHFR is essential for Candida albicans growth in vitro}

To investigate the importance of DHFR in C. albicans, we generated strains with doxycycline-repressible transcription of DFRl, which encodes DHFR [108]. One allele of $D F R I$ was deleted from a strain expressing the tetracycline responsive-transactivator protein and replaced with the $A R G 4$ selection marker [103]. The promoter of the second allele was then replaced with one of three tetracycline-repressible promoters - PTETO97, $P_{\text {TETO98, or PTETO99, which support different basal levels of transcription [102]. DFRI }}$ transcript abundance in strains of each genotype was then compared to a wild-type control (DFRI/DFRI) by RTqPCR (Figure 2-1). In the absence of doxycycline, the

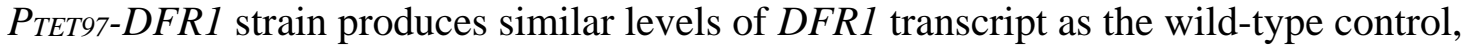
while $P_{T E T 98}-D F R I$ and $P_{T E T 99}-D F R 1$ strains overproduced $D F R l$ transcript by $\sim 4$ and $\sim 20$-fold-respectively. However, in the presence of $5 \mu \mathrm{g} / \mathrm{ml}$ doxycycline, the DFR 1 transcript abundance was dramatically reduced in strains of all three genotypes, to below that of the wild-type control (Figure 2-1). We noted that the $P_{\text {TET97-DFR } 1 \text { strain grew }}$ slowly compared to the wild-type, even in the absence of doxycycline, suggesting that Dfr1p production is insufficient, while both $P_{T E T 98-D F R I}$ and $P_{T E T 99-D F R l}$ grew at a rate 


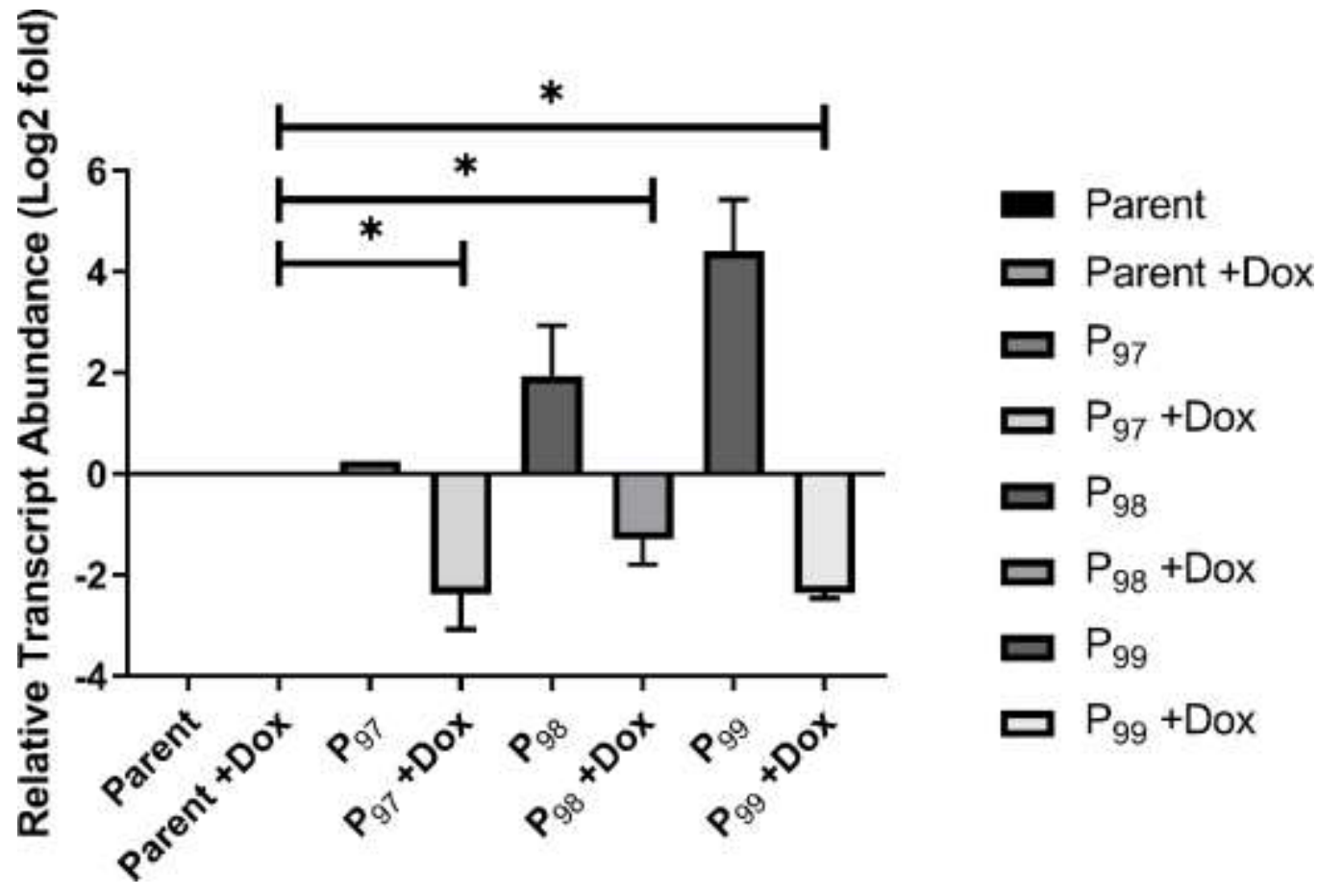

Figure 2-1. Analysis of DFR1 transcript expression in Candida albicans $P_{\text {TETO- }}$ DFR1 strains.

C. albicans strains of the indicated genotypes were grown to exponential phase in YPD medium in the presence or absence of $5 \mu \mathrm{g} / \mathrm{ml}$ doxycycline. Total RNA was extracted and $D F R 1$ transcript abundance determined by RTqPCR. DFR1 transcript abundance was normalized to that of the ACTI transcript and then expressed relative to the DFRI transcript abundance in the wild-type control (SC5314) in the absence of doxycycline. This data is the average of three biological and technical replicates. * indicates $\mathrm{P}<$ 0.0001 compared to the parent strain in the same condition using an unpaired student's $t$ test. 
comparable to wild-type (Figure 2-2). We, therefore, selected the $P_{T E T 98}-D F R 1$ strain for further study.

Next, we determined if $D F R 1$ expression is required for $C$. albicans growth in vitro. When inoculated into rich YPD medium supplemented with $5 \mu \mathrm{g} / \mathrm{ml}$ doxycycline, the $P_{\text {TET98-DFR }}$ strain grew to the same cell density as in the absence of doxycycline, and to the wild-type control (Figure 2-3A). However, when sequentially passaged in the presence of doxycycline, growth was dramatically reduced by the third passage. This suggests that DFRl is essential for $C$. albicans growth, even in nutritionally rich growth medium containing high concentrations of folate, as well as metabolites that depend upon folate for their biosynthesis. The prolonged lag between doxycycline exposure and growth suppression could indicate that cellular stores of THF permit continued growth in the absence of Dfr1p until depleted, or that the protein has a long half-life. To distinguish between these possibilities, we attempted to directly inhibit Dfr1p using methotrexate. Previous reports have indicated that MTX lacks antifungal activity against $C$. albicans [83-85], despite potent inhibition of purified fungal DHFR in biochemical assays [109]. However, we recently reported that MTX has relatively potent and on-target activity upon whole $C$. albicans cells in unbuffered YNB medium [104]. We therefore examined how MTX affected the growth of a wild-type $C$. albicans strain in YNB medium in doseresponse assays. Significant growth inhibition was observed in this medium, with a minimum inhibitory concentration ( $\mathrm{MIC}_{50}$ ) of approximately $0.78 \mu \mathrm{M}$ (data not shown), without the need for passaging. In addition, passaging of the PTET98-DFR1 strain with doxycycline in YNB medium resulted in detectable growth inhibition without the need for passaging (Figure 2-3B). These results suggest that the continued growth of the

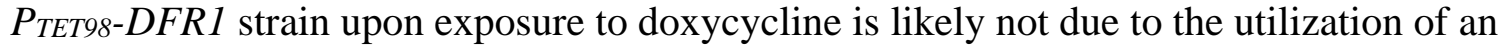
intracellular store of THF. Instead, these results are consistent with the Dfr1p enzyme having a long half-life, resulting in a long-lag before doxycycline-mediated suppression arrests fungal growth.

\section{Candida albicans has a limited capacity to utilize exogenous sources of folate}

Folate biosynthesis deficient mutants of the yeast Saccharomyces cerevisiae are unable to grow in standard lab medium $[110,111]$. However, growth can be restored by supplementing the medium with extremely high concentrations $(\geq 250 \mu \mathrm{M})$ of folinic acid (5-formyltetrahydrofolate) [112]. To determine if exogenous sources of folate are sufficient to support $C$. albicans growth in the absence of DHFR activity, we seeded SC5314 into standard unbuffered YNB medium supplemented with either folic acid, dihydrofolate (DHF), THF, or 5-methyltetrahydrofolate (5MTHF), and compared growth in the presence of MTX. Notably, standard YNB medium contains both folic acid (4.5 $\mathrm{nM}$ ) and PABA, a precursor required for folate biosynthesis. Therefore, MTX sensitivity was also tested in folate and PABA free YNB medium. The MIC $_{50}$ of MTX in standard YNB $(0.78 \mu \mathrm{M})$ was similar to that in the folate and PABA free medium, indicating that the folate present in standard YNB medium is not sufficient to affect sensitivity. Additional supplements of folic acid were completely unable to rescue $C$. albicans growth at any soluble concentration (up to $25 \mu \mathrm{M}$ ), and in fact, the MIC50 of MTX 


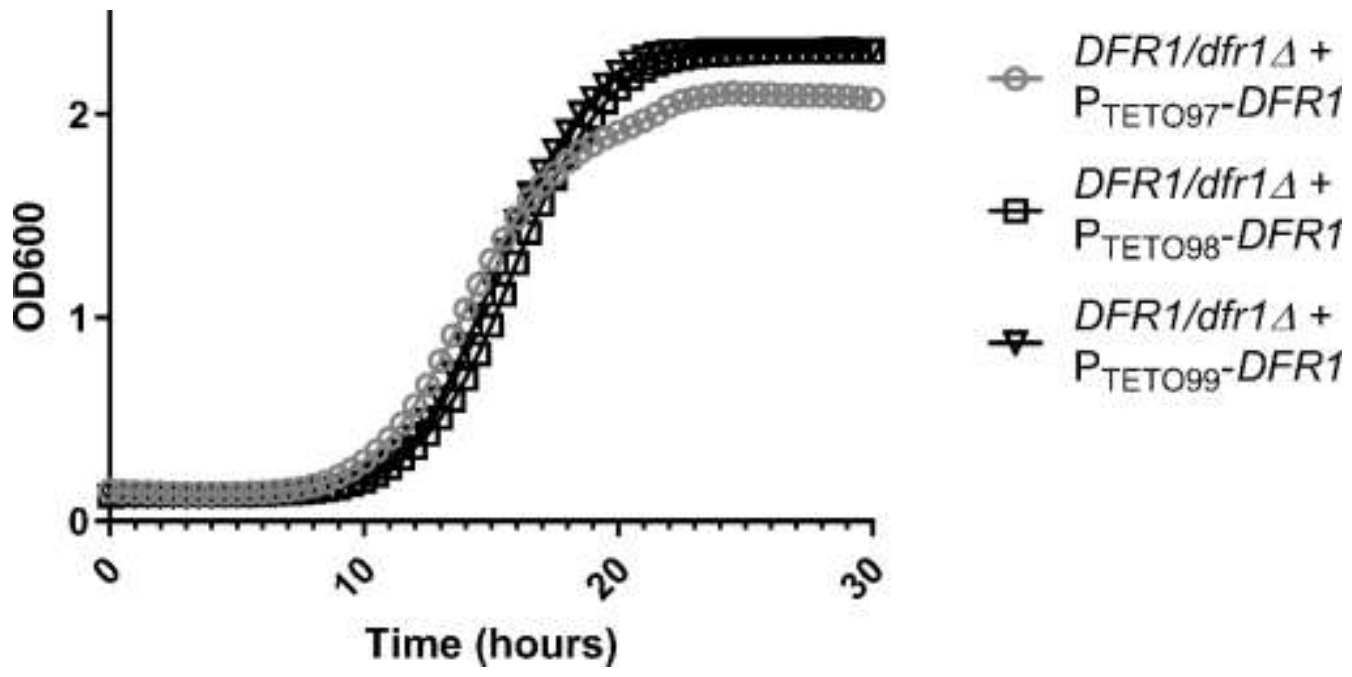

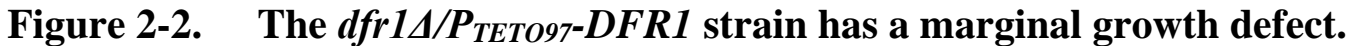

The indicated strains were sub-cultured into YPD broth at approximately $5 \times 10^{5}$ cells $/ \mathrm{ml}$ and dispensed into the wells of a 96-well plate. The plate was incubated at $30^{\circ} \mathrm{C}$ for 48

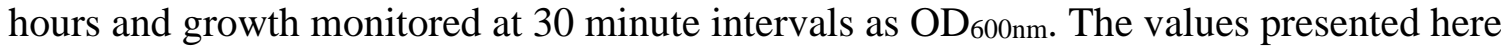
are the average of twenty-four replicates in a single experiment.
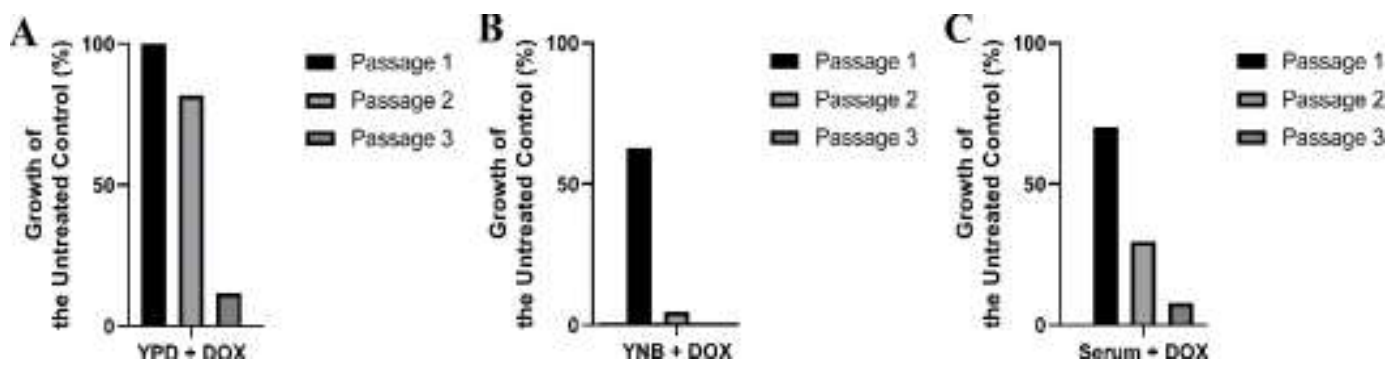

Figure 2-3. Dfr1p is essential for Candida albicans growth in vitro.

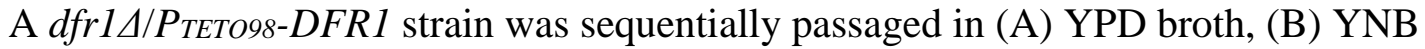
broth, or (C) fetal bovine serum, each supplemented with $5 \mu \mathrm{g} / \mathrm{ml}$ DOX. Following inoculation at approximately $5 \times 10^{5}$ cells $/ \mathrm{ml}$, each culture was incubated at $30^{\circ} \mathrm{C}$ for 24 hours and growth was measured after 24 hours as OD $600 \mathrm{~nm}$. Relative growth was then expressed as a percentage of the untreated control i.e. minus DOX. The values presented here are representative of two independent experiments. 
dropped $\sim 10$-fold to $0.078 \mu \mathrm{M}$ (Figure 2-4). Supplementing the medium with either 1 $\mu \mathrm{M}$ DHF, THF, or 5MTHF (Figure 2-5) (well above blood serum concentrations) [113115] had no effect on the MTX mediated growth inhibition of $C$. albicans. However, the addition of $250 \mu \mathrm{M}$ folinic acid to YNB was able to partially restore growth in the presence of MTX and elevated the $\mathrm{MIC}_{50}$ to $\sim 5 \mu \mathrm{M}$ (Figure 2-4). Nevertheless, even in the presence of this excess of folinic acid, MTX inhibited C. albicans growth in a dosedependent manner (Figure 2-6), indicating that the need for Dfr1p activity is not completely bypassed. Accordingly, we conclude that $C$. albicans is unable to take up and utilize sufficient quantities of exogenous folates to sustain growth in standard laboratory medium.

A combination of dTMP, adenine, histidine, and methionine, each of which requires folate for their biosynthesis, is sufficient to partially restore $S$. cerevisiae growth following treatment with antifolates [110]. However, these supplements were not sufficient to restore $C$. albicans growth in the presence of MTX (Figure 2-4). This suggests that either the provision of exogenous sources of folate-dependent metabolites is not adequate to bypass the need for de novo folate biosynthesis in $C$. albicans, or that the nutritional requirements of this species in the absence of folate are more complex than $S$. cerevisiae. Finally, we examined if $C$. albicans was able to scavenge sufficient folates or folate-dependent metabolites from blood serum to negate the need for de novo biosynthesis. The $P_{T E T 98}-D F R l$ strain was passaged for three days in $100 \%$ Fetal Bovine Serum (FBS) with or without doxycycline. In the first passage, doxycycline inhibited growth by $\sim 60 \%$, with increasing growth inhibition seen in subsequent sub-cultures (Figure 2-3C). Thus, C. albicans is unable to acquire sufficient nutrients from blood serum to bypass the need for de novo folate biosynthesis.

\section{DFR1 is essential for Candida albicans virulence in a mouse model of disseminated infection}

Next, we determined if DFRl expression is essential for $C$. albicans virulence in a mammalian host using a mouse model of disseminated candidiasis. Female BALB/c mice were split into two treatment groups: Group 1 was treated with doxycycline in a gel food formulation from 72 hours before infection, and for the duration of the experiment, Group 2 was mock treated using unsupplemented gel food. The mice were then infected with $\sim 5 \times 10^{5}$ yeast cells of $P_{\text {TET98-DFR }}$ via the lateral tail vein, and their health monitored for 12 days. Animals exhibiting significant signs of distress were euthanized and survival compared. All mice in the mock-treated group succumbed to the infection by day 7 (Figure 2-7), confirming the virulence of the $P_{T E T 98}-D F R l$ strain under nonrepressing conditions. In stark contrast, all doxycycline-treated mice survived for the duration of the experiment. On day 12, surviving animals were euthanized, kidneys extracted and homogenized, and levels of fungal colonization determined as colonyforming units (CFU). This revealed that 5 of the 8 surviving mice had undetectable levels of fungal colonization, essentially clearing the infection, with the remaining animals having extremely low levels of CFU (data not shown). To determine if suppression of Dfrlp activity is sufficient to resolve an established infection, a second experiment was 


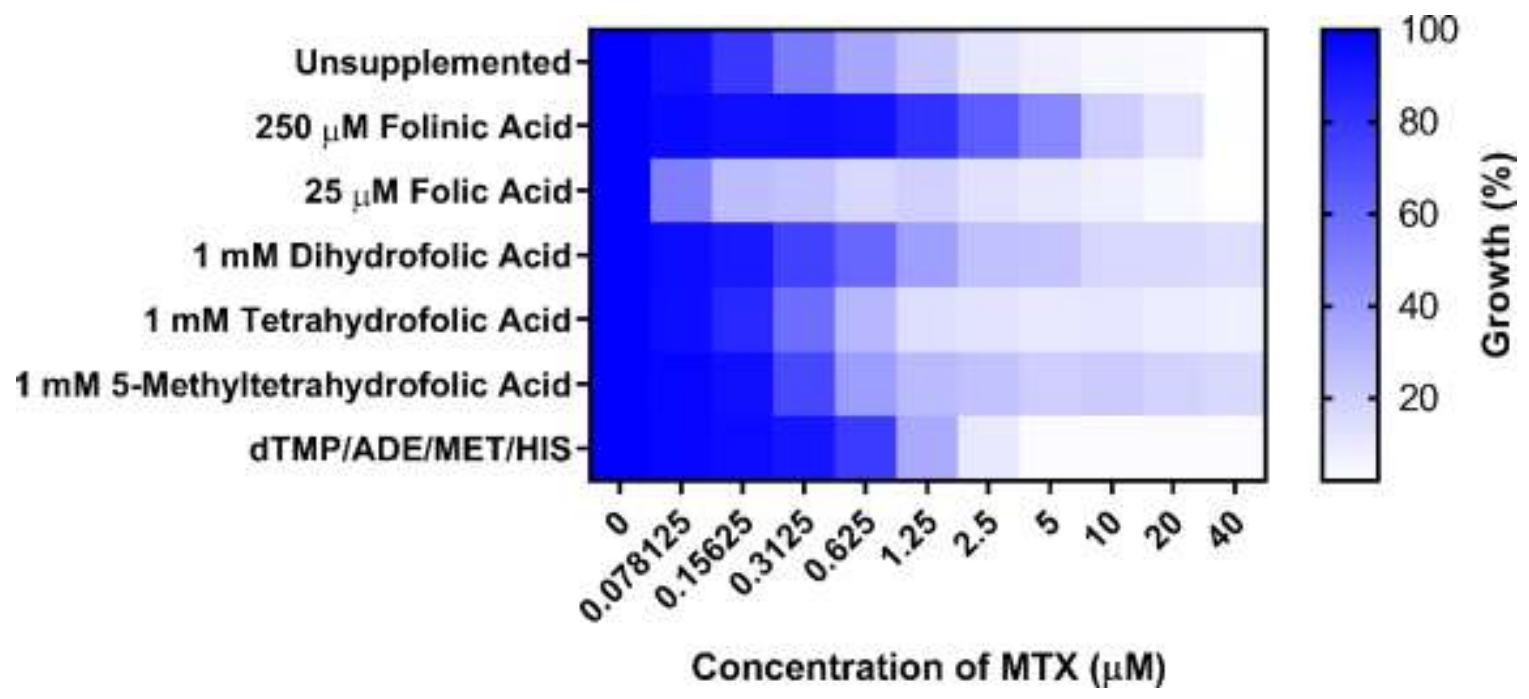

Figure 2-4. Excess folinic acid partially restores the growth Candida albicans in the presence of methotrexate.

A wild-type C. albicans strain (SC5314) was seeded at $1 \times 10^{4}$ cells $/ \mathrm{ml}$ into YNB medium without folate or PABA with the indicated supplements and increasing concentrations of MTX. After 48 hours of incubation at $30^{\circ} \mathrm{C}$, growth was quantified as OD $600 \mathrm{~nm}$, and expressed as a percentage of the DMSO treated control. The values presented here are the average of two replicates and are representative of two independent experiments

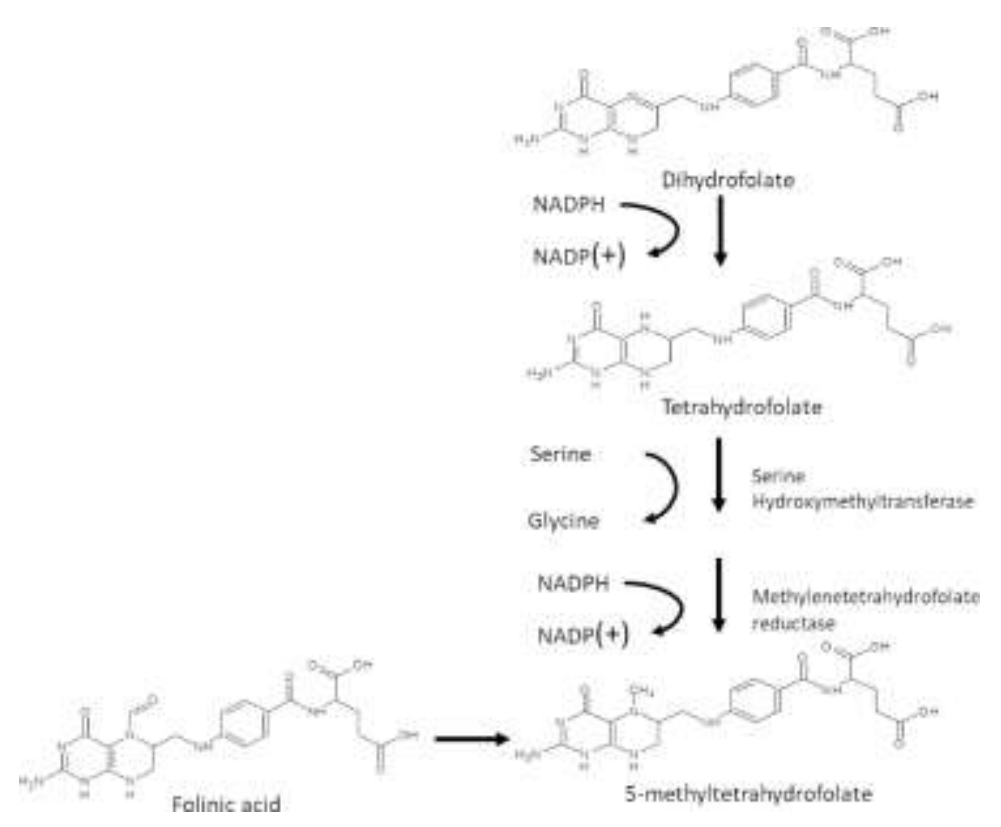

Figure 2-5. Schematic representation of the folate biosynthetic pathway. 


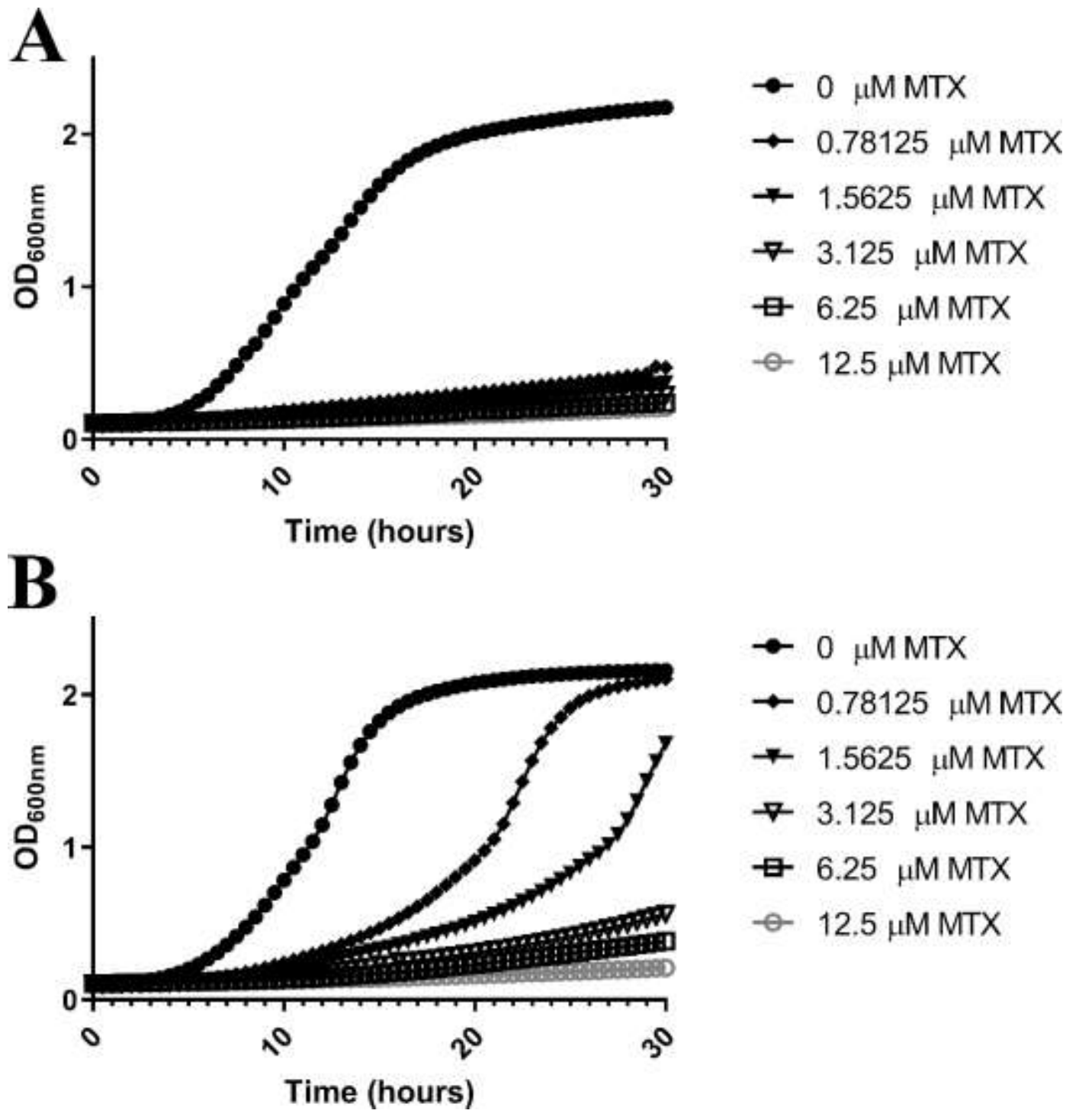

Figure 2-6. Folinic acid restores growth of Candida albicans in the presence of methotrexate.

C. albicans strain SC5314 was sub-cultured into (A) YNB medium or (B) YNB medium supplemented with $500 \mu \mathrm{M}$ folinic acid at $\sim 5 \times 10^{5}$ cells $/ \mathrm{ml}$ in the presence of increasing concentrations of MTX. These cultures were established in 96-well plates to facilitate the monitoring of growth at 30-minute intervals as $\mathrm{OD}_{600 \mathrm{~nm}}$ during a 48 hour incubation period at $30^{\circ} \mathrm{C}$. The values presented here are representative of two independent experiments. 


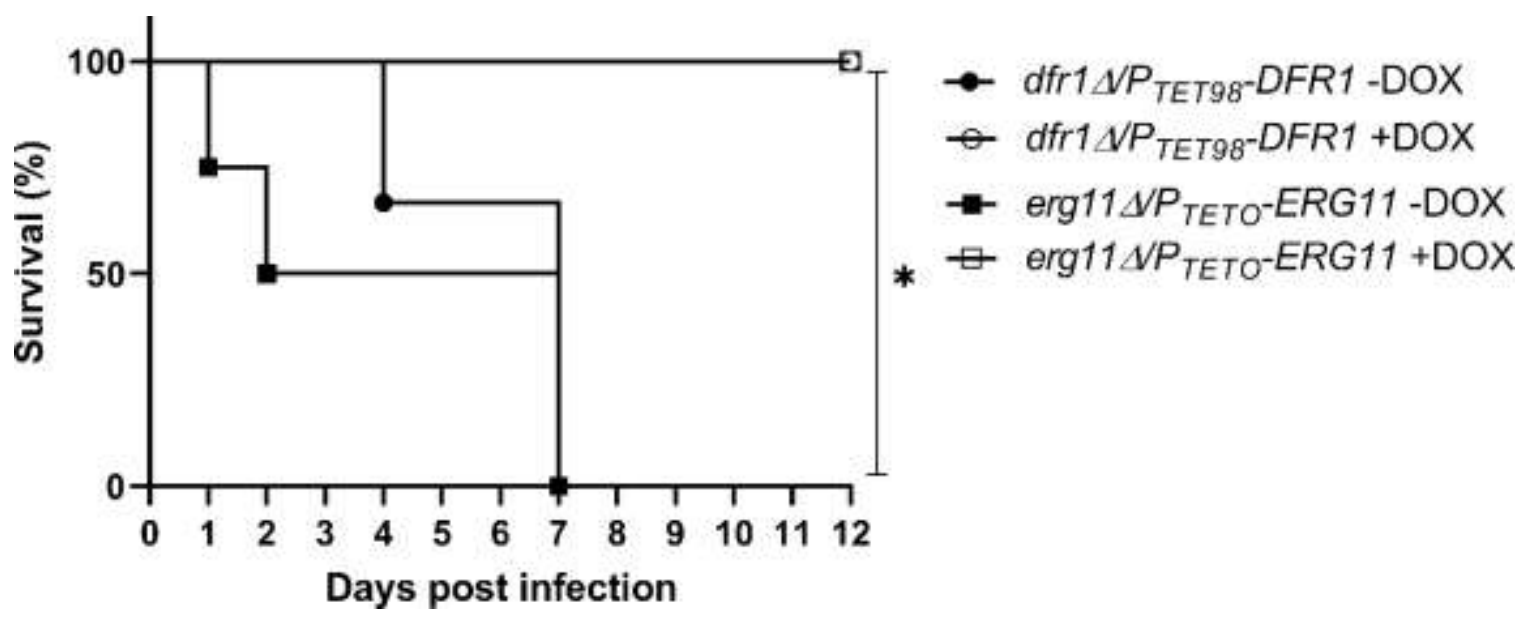

Figure 2-7. Dfr1p is essential for Candida albicans virulence in a mouse model of disseminated candidiasis.

Groups of BALB/c mice $(\mathrm{N}=6)$ were inoculated with $\sim 5 \times 10^{5} \mathrm{CFU}$ of either the

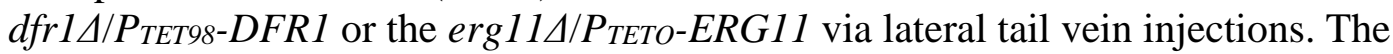
Erg11p strain was included to compare the antifungal efficacy of targeting Dfr1p with a previously validated target. The mice were monitored three times daily for 12 days, and those showing signs of distress were humanely euthanized. Mice were treated with 2 $\mathrm{mg} / \mathrm{g}$ doxycycline or mock treated with vehicle alone provided in a gel food formulation from 72 hours prior to infection and for the duration of the experiment. The survival of each group was compared using the log rank test $(*$ is a $\mathrm{P}<0.0003)$. 
performed in which mice were infected with $P_{\text {TET98-DFRI }}$ and doxycycline treatment initiated 24-hour post-infection. Again, all mice survived the duration of the experiment (to day 12 post-infection) with initial symptoms resolving within 6 days of doxycycline treatment. Half of these mice had undetectable levels of fungal colonization in their kidneys at the end of the experiment (data not shown). These data confirm that Dfrlp is essential for $C$. albicans to survive within and cause disseminated disease in a mammalian host. Furthermore, C. albicans is unable to scavenge sufficient sources of folate to survive within mammalian tissue, and de novo biosynthesis is required. Indeed the antifungal efficacy achieved upon repressing Dfr1p expression appears to be similar to that attained upon suppression of Erg11p expression. Accordingly, DHFR and likely other FOL pathway enzymes can provide potentially efficacious targets for antifungal development.

\section{The $\mathbf{p H}-d e p e n d e n t$ antifungal activity of methotrexate is likely due to differential uptake}

While MTX has been shown to inhibit fungal DHFR in cell-free biochemical assays using the purified enzyme, it has also been reported to lack antifungal activity [8385]. Given that these previous studies used RPMI $1640 \mathrm{pH} 7$ medium, we sought to account for the antifungal activity we observed in unbuffered YNB medium. We first confirmed that MTX lacked antifungal activity in RPMI $1640 \mathrm{pH} 7$ medium (MIC $50>50$ $\mu \mathrm{M})$ (Figure 2-8). Next, to determine if the antifungal activity of MTX is $\mathrm{pH}$-dependent, dose-response experiments were conducted with SC5314 in YNB medium buffered at $\mathrm{pH}$ 5 or $\mathrm{pH}$ 7. MTX inhibited C. albicans growth in $\mathrm{YNB} \mathrm{pH} 5$ with an MIC 50 of $\sim 0.78 \mu \mathrm{M}$ (Figure 2-8), but had no effect in $\mathrm{YNB}$ pH 7 ( $\mathrm{MIC}_{50}>50 \mu \mathrm{M}$ ), indicating $\mathrm{pH}$-dependent antifungal activity. Similar results were obtained in YNB medium lacking folate and PABA, buffered to either $\mathrm{pH} 5$ or $\mathrm{pH} 7$ (Figure 2-9). In contrast, the PTET98-DFRl strain was unable to grow in regular YNB, in folate and $\mathrm{PABA}$ free $\mathrm{YNB}$ medium at either $\mathrm{pH}$ in the presence of doxycycline (Figure 2-10A), or in YNB supplemented with different folates (Figure 2-10B) indicating that Dfr1p expression, and by inference de novo folate biosynthesis is essential at either $\mathrm{pH}$. These data are consistent with the previous findings of Navarro-Martinez and colleagues that demonstrated MTX does not accumulate within C. albicans yeast cells at neutral $\mathrm{pH}$ [83], and may therefore account for the $\mathrm{pH}-$ dependent antifungal activity.

Therefore we examined if the antifungal activity of MTX was affected by the most important drug efflux mechanisms in $C$. albicans. The susceptibility of a $C$. albicans cdr $1 \Delta / \Delta$ mutant, lacking an ATP-dependent ABC family transporter, as well as a tac $1 \Delta / \Delta$ mutant, lacking a zinc cluster transcription factor that activates the expression of the Cdr1p and Cdr2p drug efflux pumps [116], is not significantly different from isogenic or wild-type control strains in $\mathrm{YNB}$ at $\mathrm{pH} 5\left(\mathrm{MIC}_{50} 0.39 \mu \mathrm{M}\right)$ or pH $7\left(\mathrm{MIC}_{50}>6.25 \mu \mathrm{M}\right)$ (Figure 2-11A and B). Similarly, we found that the susceptibility of an $m d r 1 \Delta / \Delta$ mutant, lacking a major facilitator superfamily transporter, which is driven by the proton motive force at the plasma membrane, and which has been previously implicated in methotrexate efflux [117, 118], was not significantly different from $M D R 1^{+}$control strains in 


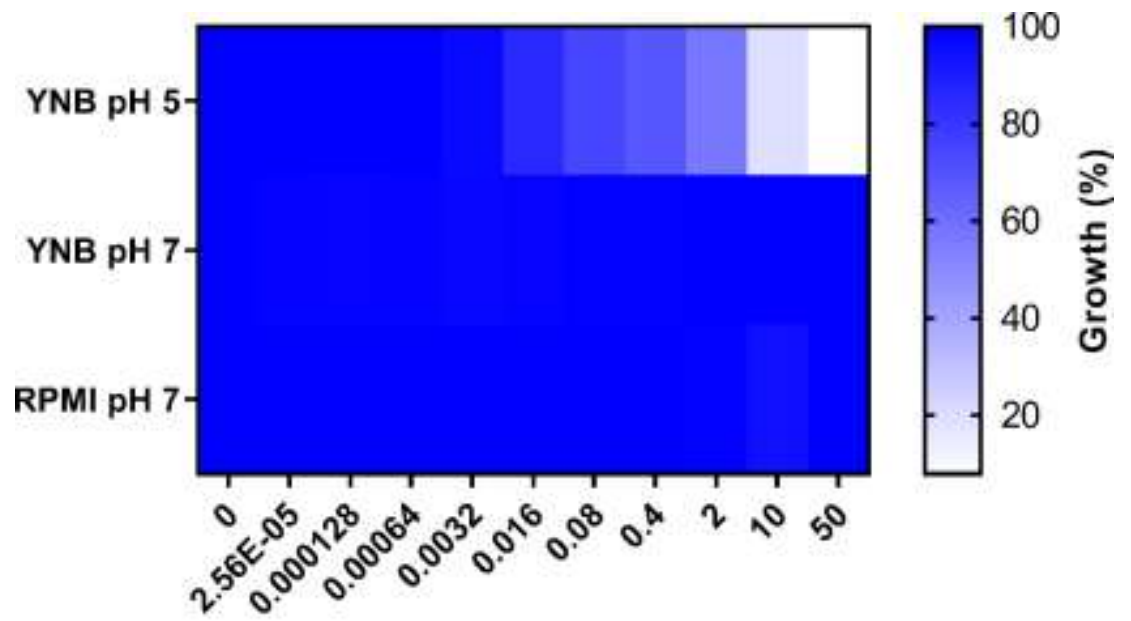

Concentration of MTX $(\mu \mathrm{M})$

Figure 2-8. Methotrexate has pH-dependent antifungal activity on Candida albicans.

Approximately $1 \times 10^{4}$ cells $/ \mathrm{ml}$ of a wild-type $C$. albicans strain was seeded into a 96well plate with increasing concentrations of MTX in YNB pH 5, YNB pH 7, and RPMI $\mathrm{pH}$ 7. After 48 hours, the OD600nm was measured and expressed as a percentage of the DMSO treated control. The results presented here are the averages of two replicates and are representative of two independent experiments.

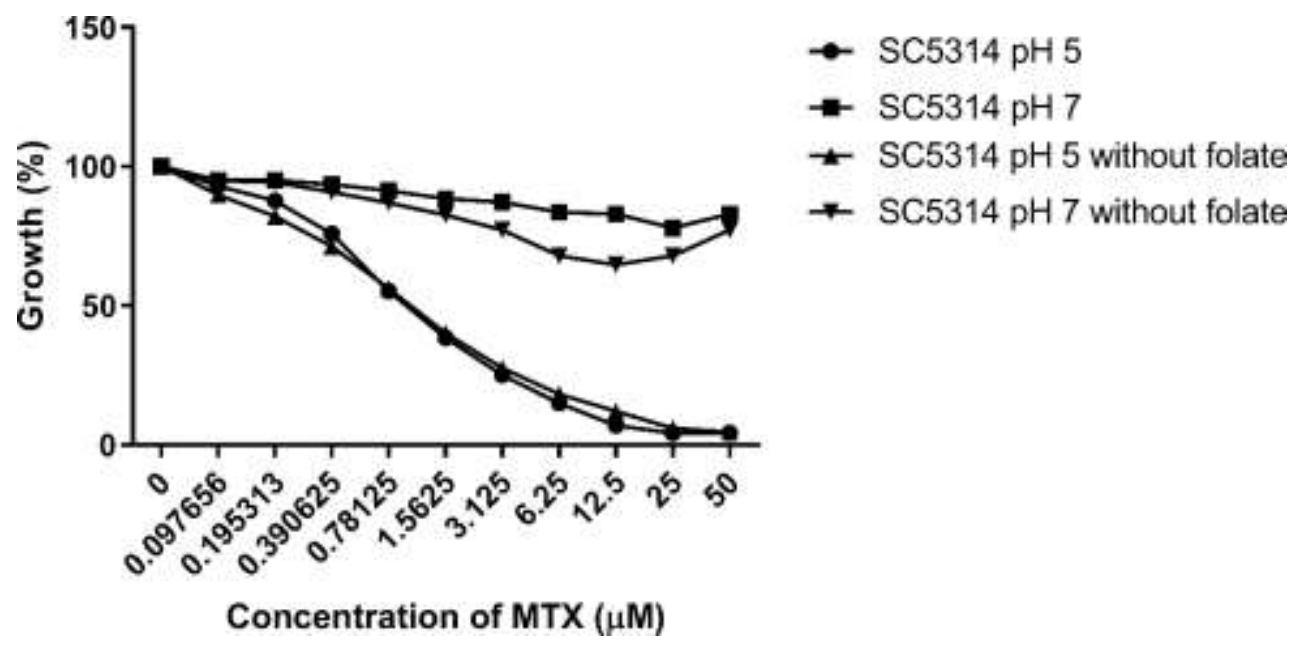

Figure 2-9. The presence of exogenous folate does not alter Candida albicans sensitivity to methotrexate.

C. albicans strain SC5314 was seeded into YNB with or without folate buffered at $\mathrm{pH} 5$ or $\mathrm{pH} 7$ to approximately $1 \mathrm{X} 10^{4}$ cells $/ \mathrm{ml}$ with increasing concentrations of MTX. After 48 hours at $30^{\circ} \mathrm{C}$, growth was quantified as OD $600 \mathrm{~nm}$ and expressed as a percentage of the untreated control wells. The values presented are the average of two replicates and are representative of two independent experiments. 


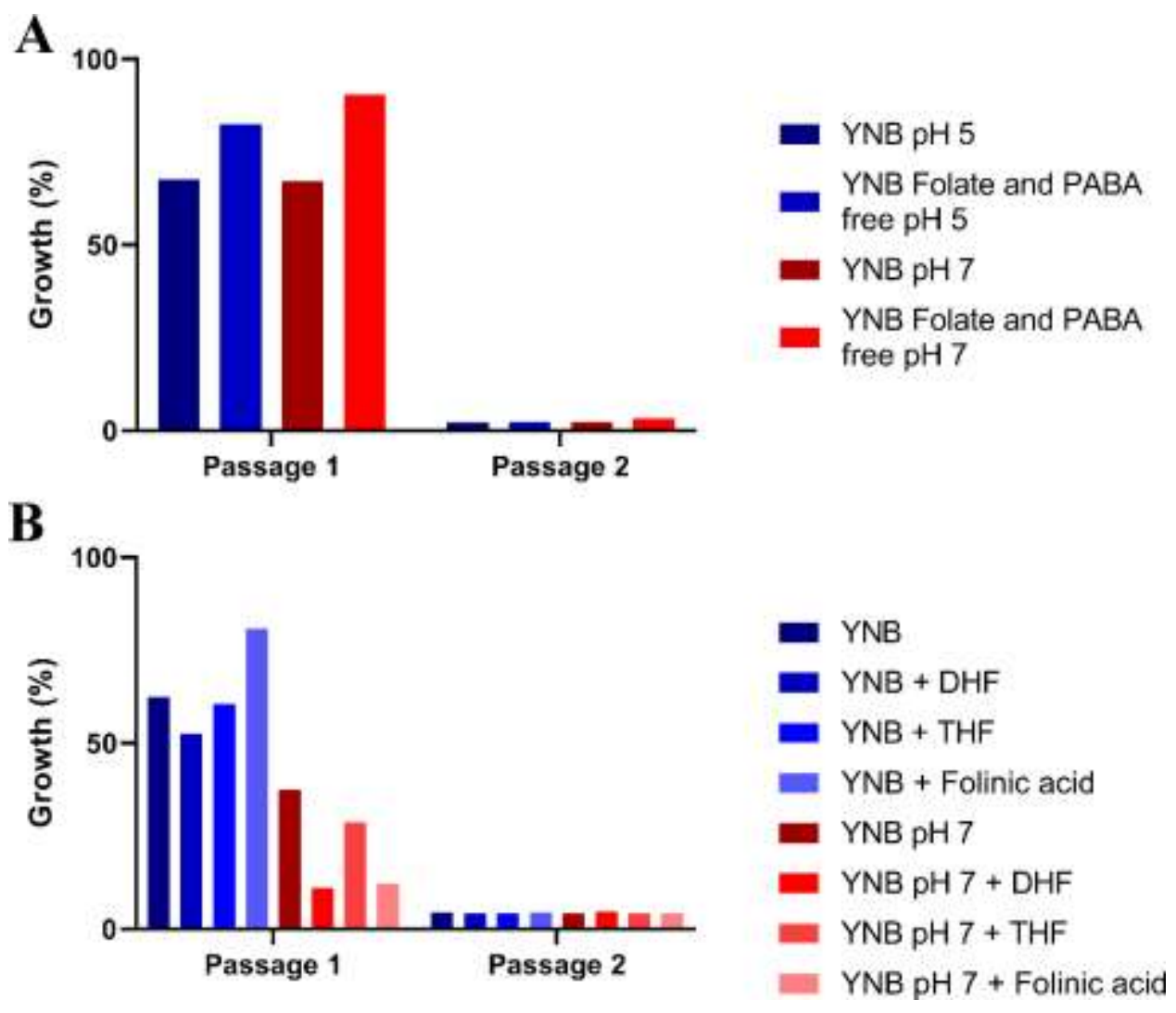

Figure 2-10. Dfr1p is essential for Candida albicans growth in both acidic and neutral conditions.

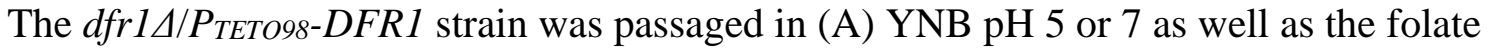
and PABA free YNB at the same $\mathrm{pH},(\mathrm{B}) \mathrm{YNB} \mathrm{pH} 5$ or 7 supplemented with different forms of folate with approximately $5 \times 10^{5}$ cells $/ \mathrm{ml}$ from the previous passage in the presence of $5 \mu \mathrm{g} / \mathrm{ml}$ doxycycline. The culture was incubated at $30^{\circ}$ in a rotating incubator and after 24 hour the OD600nm was measured. The growth was calculated as a percentage of the untreated control, i.e. without doxycycline. The values presented here are representative of two independent experiments. 
A

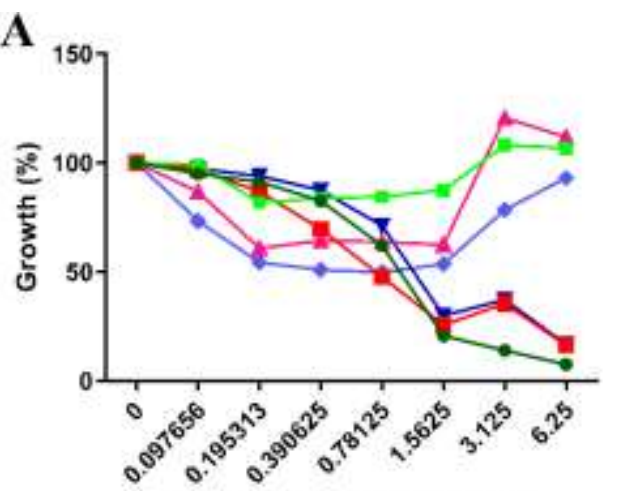

- $\mathrm{WT} \mathrm{pH} 5$

- WT pH 7

- cdr1 4/cdr1 $1 \mathrm{pH} 5$

- cdr1 $1 / \operatorname{cdr} 1 \mathrm{SpH} 7$

* $C D R 1 / C D R 1+P_{\text {TEFT }}-C D R 1 \mathrm{pH} 5$

$\rightarrow C D R 1 / C D R 1+P_{\text {TEF }}-C D R 1 \mathrm{pH} 7$

B

Concentration of MTX $(\mu \mathrm{M})$

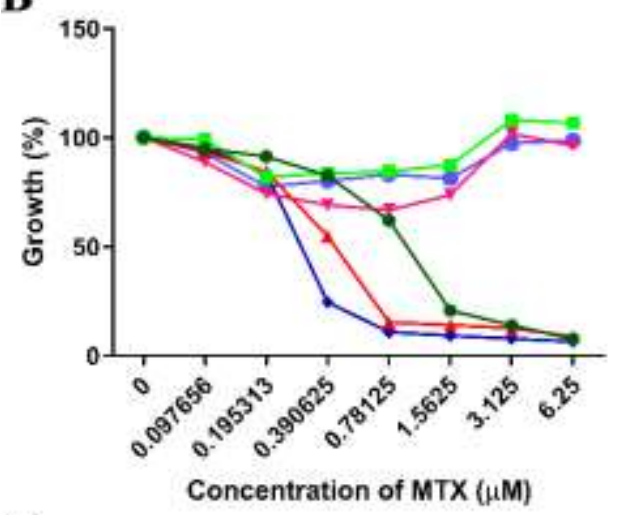

$\rightarrow$ WT pH 5

- WT pH 7

- tac1 1 /tac1 $1 \mathrm{pH} 5$

$\rightarrow$ tac1 $1 / \operatorname{tac} 1 \triangle \mathrm{pH} 7$

- TAC1/TAC1+P TEFT TAC1 pH 5

- TAC1/TAC1+P PEFT $-T A C 1 \mathrm{pH} 7$

\section{C}

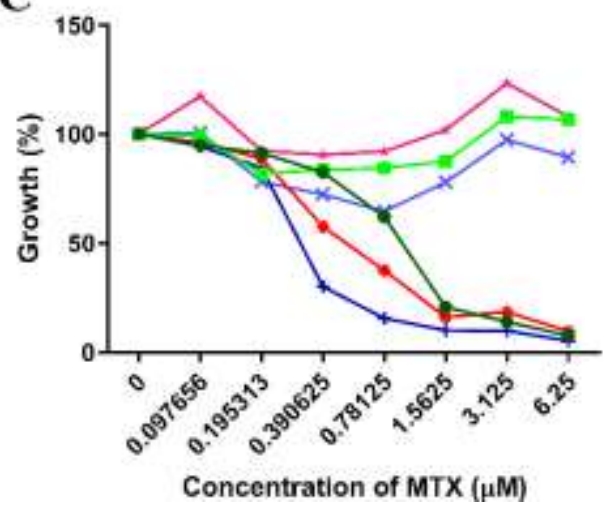

Figure 2-11. Efflux pump expression does not substantially alter Candida albicans susceptibility to methotrexate.

C. albicans cdr1 $1 / \Delta$ (A), tac1 $1 / \Delta$ (B), or $m d r 1 \Delta / \Delta$ (C) strains, or that overexpress CDR1 (A), TAC1 (B),or $M D R 1$ (C) from the $P_{T E F 1}$ promoter, were subcultured to approximately $1 \times 10^{4}$ cells $/ \mathrm{ml}$ into YNB medium at $\mathrm{pH} 5$ or $\mathrm{pH} 7$ supplemented with a range of methotrexate concentrations. After 48 hours at $30^{\circ} \mathrm{C}$, the growth was quantified by $\mathrm{OD}_{600 \mathrm{~nm}}$ and expressed as a percentage of the value for the untreated wells. The values presented here are the average of two replicates and are representative of two independent experiments. 
YNB pH 5 or 7 (Figure 2-11C). The susceptibility of strains engineered to overexpress either the Cdr1p or Mdr1p efflux pumps [116], was also unaffected. This lends further support for the hypothesis that the $\mathrm{pH}$-dependent antifungal activity of MTX is a result of differential cellular uptake or permeability.

To determine if MTX's pH-dependent activity was specific to C. albicans, we tested its antifungal activity upon other fungal species in YNB pH 5 and $\mathrm{YNB} p \mathrm{pH}$ 7. Like C. albicans, MTX showed modest and $\mathrm{pH}$-dependent growth inhibition on C. tropicalis and C. parapsilosis. In contrast, C. glabrata was insensitive at either pH (Figure 2-12).

\section{Established DHFR inhibitors are unable to inhibit Dfr1p within Candida albicans cells}

Finally, we tested a panel of additional antifolates to examine the relationship between their antifungal potency and capacity to inhibit $C$. albicans DHFR. The enzyme was purified using a $6 \mathrm{X}-\mathrm{HIS}$ tag $[119,120]$, and activity detected by measuring the conversion of NADPH to NADP ${ }^{+}$at $\mathrm{OD}_{340 \mathrm{~nm}}$ [109]. Dose-response experiments confirmed MTX to be a potent inhibitor of $C$. albicans DHFR, while trimethoprim (TMP) had no activity (Table 2-2), as previously reported [121]. While proguanil (PRO) also lacked activity ( $\mathrm{IC}_{50}>10 \mu \mathrm{M}$ ), both pyrimethamine (PYR) and pemetrexed (PTX) inhibited $C$. albicans DHFR activity. Consistent with the biochemical data, neither TMP or PRO were able to inhibit $C$. albicans growth in YNB medium buffered to either $\mathrm{pH} 5$ or 7 (MIC50 $>25 \mu \mathrm{M}$ ). PYR also failed to inhibit $C$. albicans growth at either $\mathrm{pH}$ (Figure 2-13), suggesting the compound is unable to access DHFR in whole-cells. Similar to MTX, PTX possessed antifungal activity at $\mathrm{pH} 5$, but not $\mathrm{pH} 7$ in YNB medium, presumably reflecting $\mathrm{pH}$-dependent cell permeability. It is also possible that the $\mathrm{pH}$ dependency of PTX is a result of inhibition of other enzymes, as PTX is known to inhibit thymidylate synthase and glycinamide ribonucleotide formyltransferase [122].

\section{Discussion}

While folate biosynthesis has been successfully targeted to develop antibacterial, antiprotozoal, and antineoplastic therapies, there have been relatively few efforts to develop antifungals that target this pathway. Although a combination of trimethoprim and sulfamethoxazole is used clinically to treat Pneumocystis pneumonia and Paracoccidiodes infections [82, 123], many of the most important fungal pathogens are largely insensitive to conventional antifolate drugs [83, 124, 125]. A handful of studies

have attempted to produce derivatives of conventional antifolates with enhanced antifungal potency, but have failed to yield derivatives with the requisite properties of a viable antifungal drug. For example, an entire series of sulfone compounds completely lack antifungal activity against whole $C$. albicans cells, despite potent inhibition of fungal DHPS in vitro [124]. Similarly, a large series of diaminopyrimidines, which inhibit bacterial and human DHFR, have little activity against whole $C$. albicans cells despite 

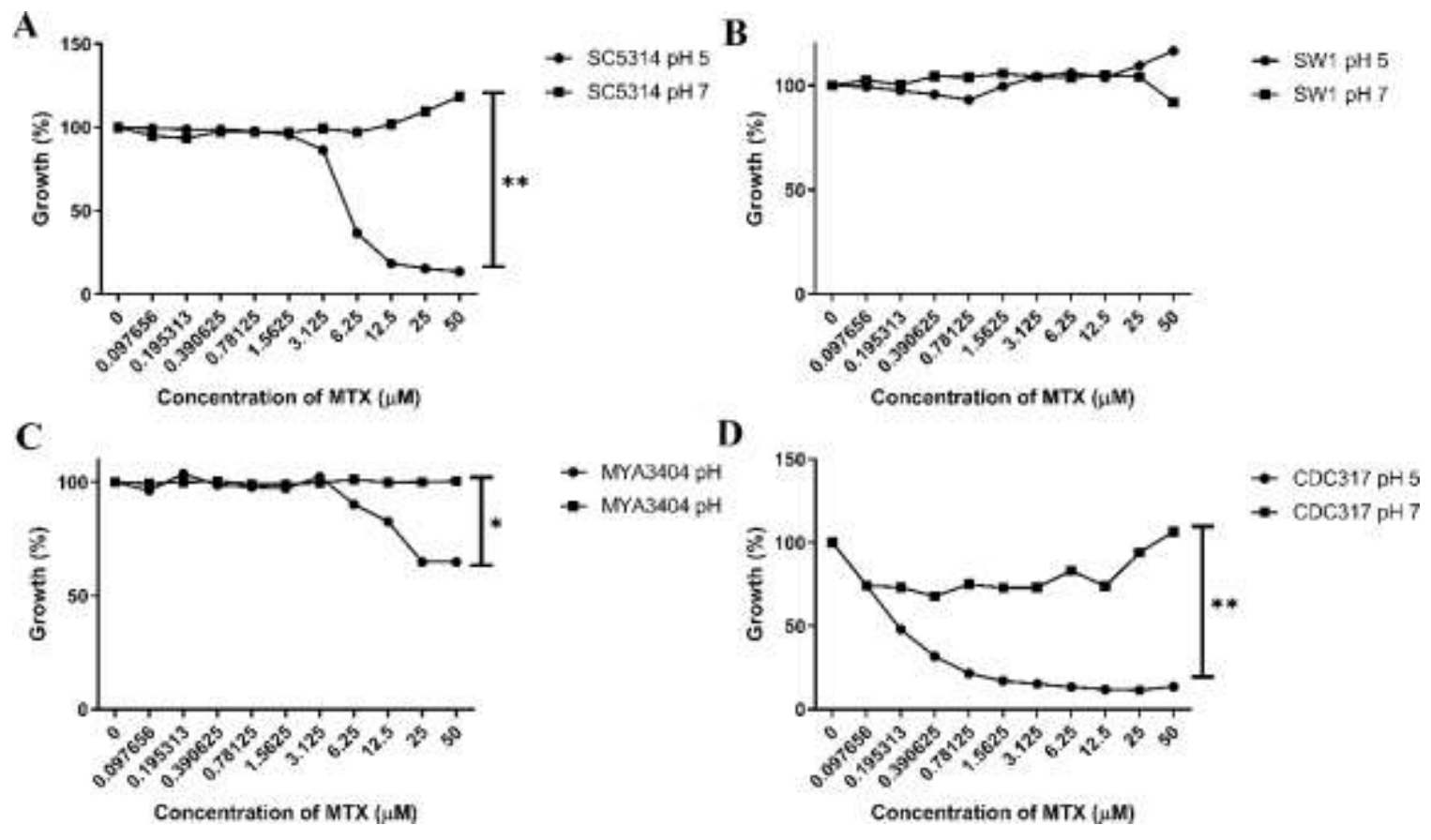

Figure 2-12. The $\mathrm{pH}$ dependent antifungal activity of methotrexate is species specific.

Strains of (A) C. albicans, (B) C. glabrata, (C) C. tropicalis, (D) C. parapsilosis were seeded into YNB medium buffered at $\mathrm{pH} 5$ or $\mathrm{pH} 7$ at approximately $1 \mathrm{X} 10^{4}$ cells $/ \mathrm{ml}$ in the presence of a range of MTX concentrations. After 48 hours at $30^{\circ} \mathrm{C}$, growth was quantified as $\mathrm{OD}_{600 \mathrm{~nm}}$ and expressed as a percentage of the untreated controls. The values presented here are the average of two replicates and are represented of two experiments. The susceptibility to MTX at the highest concentration in each $\mathrm{pH}$ was compared to the other $\mathrm{pH}$ using a two-tailed student's $\mathrm{t}$ test $(* \mathrm{P}<0.01$; $* * \mathrm{P}<0.0001)$.

Table 2-2. Antifolates inhibit the activity of Candida albicans dihydrofolate reductase.

\begin{tabular}{lc}
\hline \multicolumn{1}{c}{ Drugs } & $\mathrm{IC}_{50}(\mu \mathrm{M})$ \\
\hline Methotrexate & 0.001 \\
Trimethoprim & $>10$ \\
Pyrimethamine & 0.1 \\
Pemetrexed & 1.0 \\
Proguanil & $>10$ \\
\hline
\end{tabular}


potent inhibition of fungal DHFR in cell-free assays [124]. DHFR inhibitors based on either quinazoline [126] or pteridine [127] ring scaffolds have potent but $\mathrm{pH}$-dependent antifungal activity in vitro, which likely explains their lack of efficacy in an animal model of infection [127]. One factor complicating the interpretation of these findings is that these studies often used different culture conditions or medium. We, therefore, considered that the lack of success could be accounted for by infectious species, such as C. albicans, acquiring exogenous sources of folate from certain growth medium or under specific conditions in vitro and/or from mammalian tissue in vivo. This would bypass the need for de novo folate biosynthesis and therefore render antifolate drugs ineffective as antifungals. However, our results using the doxycycline-repressible DFRl strain revealed that DHFR, and by inference de novo production of THF, is essential for C. albicans growth in a variety of culture conditions including within blood serum as well as the mammalian host. Aside from extremely high concentrations of folinic acid, medium supplements of various forms of folate, including the DHFR product THF, were not able to restore growth upon loss of DHFR expression. Thus, DHFR is a valid and potentially efficacious target for antifungal development.

These conclusions are further supported by the fact that methotrexate has relatively potent antifungal activity against $C$. albicans in the lower $\mathrm{pH}$ range. In contrast, doxycycline-mediated suppression of DHFR activity in the $P_{T E T 98}-D F R I$ strain occurred less rapidly, requiring multiple passages to arrest fungal growth completely. This is likely a consequence of the indirect mechanism, acting through suppression of DFRI transcription, rather than direct inhibition of the fungal enzyme's activity. Nonetheless, the symptoms observed within mice infected with the PTET98-DFRl strain resolved fairly quickly upon doxycycline treatment, indicating that the fungus is highly sensitive to perturbation of THF production in vivo. We conclude that an antifungal drug acting through inhibition of DHFR has the potential to provide a highly efficacious antifungal therapy against $C$. albicans. However, given that Dfr1p is present in humans and fairly well conserved between mammals and fungi, selectivity is likely to be an issue. Interestingly, Anderson and colleagues [95, 128] described trimethoprim derivatives with an extended central linker with dramatically enhanced potency against $C$. glabrata DHFR. However, despite exhibiting selective inhibition of the fungal enzyme over the human enzyme in cell-free biochemical assays, toxicity to mammalian cells remained an issue [129]. Accordingly, assuming that inhibition of earlier steps of the folate biosynthetic pathway (FOL) results in similar antifungal efficacy, it may make more sense to target those enzymes absent from the mammalian host.

Folate synthesis starts with the conversion of GTP to dihydroneopterin triphosphate by GTP cyclohydrolase I (Fol2p), which is subsequently condensed with para-aminobenzoic acid (PABA) to form dihydropteroate by the sequential action of dihydroneopterin aldolase (DHNA), hydroxymethyldihydropterin pyrophosphokinase (HPPK), and dihydropteroate synthase (DHPS) [130]. In yeast, the latter three enzyme activities are provided by a single trifunctional protein, Fol1p, while in filamentous fungi HPPK and DHPS form a bifunctional enzyme [131]. Dihydrofolate synthase (DHFS; Fol3p) adds a single L-glutamate residue to produce DHF, which is subsequently reduced by DHFR (Dfr1p) to produce THF that can accept 1C substituents. Notably, mammals 
completely lack four of the core enzymes required to produce folate, DHNA, PPPK, DHPS (Follp), and DHFS (Fol3p). In the non-infectious yeast Saccharomyces cerevisiae, the FOL1 and FOL3 genes are both essential for viability under normal culture conditions $[130,131]$. A recent large-scale study using transposon mutagenesis also predicted FOL1 to be essential in $C$. albicans [133], and an unpublished study has indicated that the bifunctional FOL1 ortholog is essential in A. fumigatus [134]. Nonetheless, these enzymes are surprisingly poorly studied in infectious fungi, or indeed $S$. cerevisiae.

The fact that the doxycycline-repressible $D F R I$ strain is unable to grow in any growth medium tested (including those supplemented with THF) under repressing conditions is consistent with conventional antifolates lacking activity upon the target enzyme in whole cells, rather than an inherent capacity of $C$. albicans to bypass the need for folate biosynthesis. Given that both sulfa-based DHPS inhibitors and various DHFR inhibitors have demonstrated relatively potent inhibition of the respective fungal enzymes in cell-free assays $[109,121,124,127,128]$, the most likely challenge in deriving antifungals that target folate biosynthesis is achieving sufficient intracellular accumulation. In the case of mammalian cells, DHFR inhibitors such as MTX, which are structurally related to folic acid itself, enter primarily through the endogenous folate transport system [135]. While the lack of a known folate transport system, in theory, renders fungi vulnerable to antifolates, it simultaneously raises challenges in promoting the uptake of folate analogs. We consider two possible explanations for the $\mathrm{pH}$-dependent antifungal activity of MTX. First, fungi could potentially encode a $\mathrm{pH}$-dependent folate transport system, that enables MTX uptake in more acidic conditions. However, we do not favor this explanation as such a system would be expected to facilitate the uptake of folates in the medium themselves simultaneously, and thus bypass the need for de novo synthesis. Second, and perhaps more likely, MTX uptake in fungi could occur solely through passive diffusion across the plasma membrane, with the $\mathrm{pH}$ dependence simply reflecting the ionization state of MTX. The pKa of MTX is 4.7 [136]; thus, deprotonation of the associated carboxylic acid group of MTX at higher $\mathrm{pH}$ would confer a negative charge that may render it membrane impermeable. Either way, we found that the bestcharacterized drug efflux mechanisms in C. albicans, the proton-driven Mdr1p, and ATP driven Cdr1p and Cdr2p transporters, had little impact on the sensitivity of strain SC5314 to MTX, and therefore do not explain the lack of antifungal activity at neutral $\mathrm{pH}$. Thus, existing drugs that inhibit DHFR cannot provide antifungals for two main reasons: First, the fungal enzyme is insensitive to these drugs, presumably due to evolutionary divergence from the DHFR enzyme in their intended target species. For example, while trimethoprim apparently possesses some antifungal activity against Pneumocystis jirovecii given its clinical efficacy, $C$. albicans DHFR is insensitive [121]. Second, an inability to access DHFR within the intact fungal cell, issues that are likely determined by membrane permeability. This likely accounts for the lack of antifungal activity of pyrimethamine and the $\mathrm{pH}$-dependent activity of MTX and pemetrexed.

C. glabrata was insensitive to MTX at any $\mathrm{pH}$, which indicates that either the Dfr1p enzyme is structurally distinct or this species has a different acquisition or permeability of folates. Previous studies have shown that the structure of Dfr1p is conserved between $C$. glabrata and C. albicans [137]. However, this same study 
established that while an inhibitor could inhibit both CgDfr1p and CaDfr $1 \mathrm{p}$, this enzyme inhibition did not always correlate with fungal growth inhibition, nor did the inhibition correlate between species [138]. Therefore, we conclude that the differential in MTX pHdependent activity may be a result of differential uptake of antifolates.

In summary, our results demonstrate that the de novo synthesis of THF is absolutely required for $C$. albicans to cause disease within the mammalian host and that DHFR is a valid and potentially efficacious target for antifungal development. However, given the structural similarity of fungal and mammalian DHFR and the difficulties in producing DHFR inhibitors that are active upon whole fungal cells, we propose efforts to exploit the FOL pathway for antifungal development should focus upon the biosynthetic enzymes that have not yet been the subject of significant investigation, and which are completely absent from mammals. In addition, this may enhance the chances of discovering novel antifolate scaffolds that have activity upon whole fungal cells. 


\section{CHAPTER 3. TARGET ABUNDANCE-BASED FITNESS SCREENING (TAFIS) FACILITATES RAPID IDENTIFICATION OF TARGET-SPECIFIC AND PHYSIOLOGICALLY ACTIVE CHEMICAL PROBES ${ }^{2}$}

\section{Introduction}

The process of drug discovery, development, and approval often takes more than a decade, with a recent report estimating the associated costs at over $\$ 2.5$ billion [62]. As such, there is an urgent need to increase the efficiency with which new experimental therapeutics are discovered and developed. This is especially true for antifungal development, with the newest class of antifungal drugs, the echinocandins, taking 30 years to reach patients [56]. High attrition rates at both the preclinical and clinical stages prolong this timeline and greatly increase the associated costs. Many failures occur because hit compounds selected from the initial chemical screening steps lack the qualities of effective therapies, such as target specificity or membrane permeability. This is a direct result of the significant limitations of the two conventional drug screening approaches. Target-based screens require the development of a biochemical assay that is utilized to identify inhibitors or activators of a purified target protein. While this is often an effective strategy to identify potent chemical modulators of the target protein, many of the compounds identified have poor membrane permeability or do not engage the target protein in its native environment, i.e. whole cells. As such, many hits from target-based screens lack activity upon whole cells or target specificity and consequently have offtarget effects and/or toxicity. This necessitates costly and often unsuccessful efforts to improve cell permeability and/or selective target engagement. Thus, hits obtained through target-based screens have not translated well into clinically useful antimicrobial agents [64]. Furthermore, many proteins are not amenable to purification or high-throughput (HTP)-compatible biochemical assays, and thus are not well suited for target-based chemical screens.

Whole-cell-based screens identify compounds that induce or correct a diseaserelevant phenotype (e.g. inhibition of microbial growth), but the molecular target and mechanism of action (MOA) of hit compounds are usually unknown. Identification of the molecular target requires a substantial investment of time and resources, without which further development and optimization of promising lead compounds toward a viable therapeutic is severely restricted $[65,66]$. Thus, with either strategy, the identification of pharmacologically-active agents that act via a defined MOA is a multistep process. Significant increases in the efficiency of drug discovery and development can be achieved through the rapid identification of physiologically-active hits that act upon a specific target protein or pathway within living cells. Furthermore, the elimination of

\footnotetext{
${ }^{2}$ Modified from final submission with open access permission. Butts A, DeJarnette C, Peters TL, Parker JE, Kerns ME, Eberle KE, Kelly SL, Palmer GE. 2017. Target abundance-based fitness screening (TAFiS) facilitates rapid identification of target-specific and physiologically active chemical probes. mSphere 2:e0379-17. https://doi.org/10.1128/mSphere.00379-17. [140]
} 
compounds that lack the requisite target specificity during primary screening would also yield dramatic cost and time savings.

The purpose of this study was to establish and validate an innovative HTP screening strategy that can dramatically improve the efficiency with which target-specific and physiologically-active chemical probes are identified. Our approach is a type of target-based whole-cell screen (TBWCS), which identifies small molecules that functionally interact with a selected target protein within intact cells [71]. Like previous TBWCS, our method, which we have termed target abundance-based fitness screening (TAFiS), relies on the fundamental principles of chemical genetics- that altering the abundance of a target protein usually affects a cell's susceptibility to chemical modulators of that target [139]. However, TAFiS also integrates the principles of competitive fitness to enhance the efficiency and sensitivity of the screening assay and incorporates fluorescent protein (FP) tags to facilitate quantitative measurement of the chemical-target interaction. To provide proof of principle, we have developed the tools and methodology necessary to conduct TAFiS in the prevalent human fungal pathogen Candida albicans. We also validated TAFiS using the previously validated dihydrofolate reductase (Dfr1p) (Chapter 2). However, the approach we have developed is broadly applicable and can theoretically be applied to almost any target and in any genetically tractable microbe.

\section{Materials and Methods}

\section{Growth conditions}

C. albicans was routinely grown on yeast extract-peptone-dextrose (YPD) medium agar plates at $30^{\circ} \mathrm{C}$, supplemented with $50 \mu \mathrm{g} / \mathrm{mL}$ uridine for $u \mathrm{ra} 3 \Delta / \Delta$ strains. Selection of $C$. albicans transformants was carried out on minimal YNB medium (6.75 $\mathrm{g} / \mathrm{L}$ yeast nitrogen base without amino acids, $2 \%$ dextrose, $2 \%$ Bacto agar) supplemented with the appropriate auxotrophic requirements described for $S$. cerevisiae [98] or with 50 $\mu \mathrm{g} / \mathrm{mL}$ uridine.

\section{Plasmid construction}

Plasmid pLUX [140] was kindly provided by Dr. William Fonzi (Georgetown University), pMG2254 [142] and pMG1648 [143] were acquired from the fungal genetics stock center, pENO1-dTom-NATr [144] was acquired from Addgene, pGateway-TagBFP [145] was acquired from Evrogen, pFA-GFP $\gamma$ [146] was kindly provided by Dr. James Konopka (Stony Brook University), pGEMHIS1, pDDB57 and pRSARG4 $\triangle$ SpeI [99, 103] were kindly provided by Dr. Aaron Mitchell (Carnegie Mellon University). The $C$. albicans expression vectors pKE1 $\left(P_{A C T 1}\right)$ [146] and pKE3 ( $\left.P_{E N O 1}\right)$ [147] have been previously described. To construct the expression vector pKE4, $565 \mathrm{bp}$ of the $C$. albicans TEF1 promoter $\left(P_{T E F 1}\right)$ was amplified from strain SC5314 using primers TEF1prF-KpnI 
and TEF1prR-SalI, cut with Kpn I and Sal I, and inserted between the same sites of pKE1 to replace the $A C T 1$ promoter. This formed an expression cassette consisting of $P_{T E F 1}$, a multiple cloning site (Sal I-Cla I-Eag I-Mlu I-Sph I), followed by 414 bp of the ADH1 3' UTR sequence. This vector harbors the URA3 selection marker of $C$. albicans, and can be targeted for integration at and fully reconstitution the URA3-IROI locus of all CAI4/BWP17 derived lab strains of $C$. albicans. The pAR8 expression vector was made by amplifying the $P_{T E F 1-M C S-A D H 1-3}$ 'UTR cassette from pKE4 with primers TEF1prFSpeI and ADH1-3'UTRR-SacI, and cloning the resulting product between the Spe I and $S a c$ I sites of pRSARG4 $\Delta$ SpeI. This expression vector can be targeted for integration at the ARG4 locus of $C$. albicans.

GFP $\gamma, Y F P, m C h e r r y$, and dTomato $(d T O M)$ coding sequences were amplified from $\mathrm{pFAGFP} \gamma$, pYFPURA3, pMG2254 and pENO1-dTom-NATr plasmids with primers engineered to incorporate $\mathrm{Sal}$ I and Mlu I sites either side of the coding sequence. Each was subsequently cloned between the Sal I and Mlu I sites of plasmids pKE4 and pAR8. The coding sequences for tagBFP [8], cerulean (CER) [14], $\varphi Y F P$ [15], ZsYellow [16], and mPlum [17] were codon optimized according to the codon bias of a subset of highly expressed C. albicans proteins using the OPTIMIZER one codon/one AA program [18]. Synthetic sequences incorporating $S a l$ I and $M l u$ I sites either side of each optimized coding sequence were produced by IDTDNA, amplified from the supplied DNA template using primers AMPF1 and AMPR1, and cloned between the Sal I and Mlu I sites of pKE4 and pAR8. Plasmids pKE1:DFR1, pKE3:DFR1, and pKE4:DFR1 was made by amplifying the DFRI ORF from SC5314 genomic DNA with DFR1ORFF-SalI and DFR1ORFR-MluI primers, and cloning the resulting product between Sal I and Mlu I sites of pKE1, pKE3, or pKE4. All plasmid constructs were sequence verified to ensure the correct cloning of products and absence of unintended mutations.

\section{Candida albicans strain construction}

BWP17 [103], and CAI4 [149], were kindly provided by Aaron Mitchell (Carnegie Mellon University) and William Fonzi (Georgetown University), respectively. C. albicans was transformed with DNA constructs using the lithium acetate procedure [100]. Gene deletion strains were constructed using the PCR-based approach described by Wilson et al. [103]. All pKE3 and pKE4 based vectors (URA3 selection marker), including pKE1:DFR1, pKE3:DFR1, pKE4: DFR1 and the pKE4 based FP expression constructs were cut with Nhe I prior to transformation of $u \mathrm{ra} 3 \Delta / \Delta$ recipient $C$. albicans strains, to target integration and fully restore the URA3-IRO1 locus. All pAR8 based vectors ( $A R G 4$ selection marker), including the pAR8 based FP expression constructs were cut with $C l a$ I prior to transformation of $\arg 4 \Delta / \Delta$ recipient $C$. albicans strains, to target integration and restoration of the $A R G 4$ locus.

The DFR1 gene deletion cassette was amplified using primer set DFR1DISF and DFR1DISR and plasmid pGEMHIS1 (HIS1 selection marker) as template and transformed into BWP17. Correct integration of the gene deletion cassette to replace one DFR1 allele was confirmed by diagnostic PCR, using primers HIS1INTR2 and 
DFR1AMPR-SacI as well as HIS1INTF2 and DFR1AMPF-KpnI. To further suppress the expression of the remaining DFRl allele, we applied an approach known as Decreased Abundance by mRNA Perturbation (DAmP) [150] to destabilize the mRNA transcript. The 3'UTR of the remaining DFRl allele was targeted for disruption using a deletion cassette amplified using DFR1DMPF2 and DFR1DMPR2 with pDDB57 as template. The DFR1/dfr1A:HIS1 heterozygous strain was transformed with the DAmP cassette, transformants selected, and the absence of an intact DFR1 3'UTR confirmed by the absence of a PCR product following amplification with primer pair DFR1DETF and DFR1AMPR-SacI. Correct insertion of the pDDB57 (URA3) cassette into the 3' UTR of DFR 1 was also confirmed by the presence of a 968 bp PCR product using primer pair DFR1DETF and URA3INTR2. Oligonucleotides used in this study are included in Table 3-1.

\section{Comparison of FP tag brightness}

Several $C$. albicans transformants expressing each FP tag were grown overnight in YPD medium at $30^{\circ} \mathrm{C}$, diluted to approximately $5 \times 10^{3}$ cells $/ \mathrm{mL}$ in YNB medium and $200 \mu \mathrm{l}$ of each cell suspension transferred into the wells of a round-bottom 96-well plate. Following incubation at $30^{\circ} \mathrm{C}$ for 48 hours, fluorescence intensity at the appropriate wavelengths and OD600nm were measured using a Cytation 5 plate reader (BioTek Instruments, Inc.). Excitation and emission wavelengths used for tagBFP, CER, GFP $\gamma$, YFP, $\varphi$ YFP, ZsYellow, dTOM, mCherry, and mPlum were 402/457, 433/475, 488/507, $510 / 531,529 / 550,533 / 558,554 / 581,587 / 612$, and 590/649 respectively using a $9 \mathrm{~nm}$ bandwidth for both excitation and emission wavelengths. Fluorescence intensity was then normalized to growth $\left(\mathrm{OD}_{600 \mathrm{~nm}}\right)$ and expressed relative to background fluorescence, as measured in an isogenic, vector alone control strain. For the purpose of pooled experiments involving both $\varphi$ YFP and dTOM tagged strains, suboptimal wavelengths of $560 / 590$ with $9 \mathrm{~nm}$ bandwidth was used for dTOM to minimize spectral overlap.

\section{Antifungal susceptibility testing}

Stock solutions of fluconazole, miconazole, voriconazole, terbinafine, methotrexate, and caspofungin (Sigma-Aldrich) were prepared at $10 \mathrm{mM}$ in DMSO, and diluted as needed in the same solvent. Each $C$. albicans strain was grown overnight in YPD medium at $30^{\circ} \mathrm{C}$, diluted to 1 X $10^{4}$ cells $/ \mathrm{mL}$ in YNB medium and $100 \mu \mathrm{l}$ of each cell suspension transferred to the wells of a round-bottom 96-well plate. An additional $100 \mu \mathrm{l}$ of YNB medium containing $2 \mathrm{X}$ the final desired concentration of each drug was then added to each well. The final concentration of DMSO was $0.5 \%$ for all treatments. Plates were then incubated at $30^{\circ} \mathrm{C}$ before growth was quantified after 24 and 48 hours by measuring OD600nm using a Cytation 5 plate reader (BioTek Instruments, Inc.). The growth of each strain at each drug concentration was then expressed relative to the minus drug (DMSO alone) control. The multi-strain competition based experiments were

performed as described above except the individual FP tagged strains were diluted to $1 \mathrm{X}$ 
Table 3-1. Oligonucleotides used in Chapter 3.

\begin{tabular}{|c|c|}
\hline Primer & Sequence 5 ' $\rightarrow 3$ ' \\
\hline ACT1prF-BamHI & TCAGGATCCCCAGCCTCGTTTATAATAAACTTAGTC \\
\hline ACT1prR-EagI & TCACGGCCGTTTGAATGATTATATTTTTTTAATATTAATATCGAG \\
\hline ACT1prSEQF & CACCAAGATTTATTGCCAACG \\
\hline ADH1-3'UTRR-SacI & TCATCAGAGCTCGAAAACTTGAAACTTGAAAACACC \\
\hline AMPF1 & TGCTGAAGCTTCTTTGAGTGG \\
\hline AMPF2 & TTCAATATTCAATGGATGAGTCCCGGCCGGTCGAC \\
\hline AMPR1 & AGCAAAGAACATGGCAGCACC \\
\hline AMPR2 & CACTCCCATCATGATCATGATTACCGACGCGT \\
\hline ARGINTF2 & AAGCTAGTGTGGAAAGAAGAG \\
\hline ARGINTR2 & AATGACTGAATTATGTCGGTC \\
\hline DFR1AMPF-KpnI & TCAGGTACCTTGAGTTGTGGACTTAGTGGG \\
\hline DFR1AMPR-SacI & TCAGAGCTCCGACTTTGGAGAGAGAACAGC \\
\hline DFR1DETF & GACGTCACCACCAGAACAACG \\
\hline \multirow[t]{2}{*}{ DFR1DISF } & TTCTTGTTTGATTGAAAAAAAACTTTCACCACTAATCTAGAACTTC \\
\hline & AGGAATAGACAACAGTTTTCCCAGTCACGACGTT \\
\hline \multirow[t]{2}{*}{ DFR1DISR } & TTATTTTCTTGTCCATAGCGTATAATTATAGGTAAAATCACCTTCCT \\
\hline & TGATATCGTCTTTGTGGAATTGTGAGCGGATA \\
\hline \multirow[t]{2}{*}{ DFR1DMPF2 } & TATTAGAAGACGATATCAAGGAAGGTGATTTTACCTATAATTATAC \\
\hline & GCTATGGACAAGAAAATAATGTGGAATTGTGAGCGGATA \\
\hline \multirow[t]{2}{*}{ DFR1DMPR2 } & AACATCRCGAATGAAGGTGTTTGAATAAGTTATTCAATGGCCCTCT \\
\hline & TTTTTTGTTTGTATATTTTGTTTTCCCAGTCACGACGTT \\
\hline DFR1ORFF-SalI & TCAGTCGACAGAACTTCACGAATAGACAAC \\
\hline DFR1ORFR-MluI & TCAACGCGTATAAGTTATTCAATGGCCCTC \\
\hline ENO1prF2-BamHI & TCAGGTACCGTTACAATTGAGATGGAAAGCG \\
\hline ENO1prR-EagI & TCA \\
\hline ENO1prSEQF & СТАТТTСТTТССТTТТСТССC \\
\hline
\end{tabular}

Engineered restriction enzyme sites are underlined. 
$10^{5}$ cells/mL, mixed 1:1:1 to create the three-membered expression pools, and $1: 1: 1: 1$ to create the four-membered pools, before they were dispensed into the 96-well plates. Following incubation at $30^{\circ} \mathrm{C}$ for $48 \mathrm{hrs}$, OD $600 \mathrm{~nm}$ and fluorescence intensity was measured as described above. The relative growth of each strain in the presence of drug was then expressed as a percentage of the growth of the same strain in the minus drug (DMSO) control co-culture, as measured by fluorescence intensity of the corresponding FP tag.

\section{Stress phenotypes}

Each C. albicans strain was grown overnight in YPD medium at $30^{\circ} \mathrm{C}$, the cell density adjusted to $10^{7}$ cells $/ \mathrm{mL}$ in sterile water, and serial 1:10 dilutions performed in a 96-well plate. Cell suspensions were then applied to agar plate surfaces using a sterile multipronged applicator. Resistance to temperature stress was determined on YPD medium agar at 37 and $42^{\circ} \mathrm{C}$; resistance to osmotic stress was determined on YPD medium agar supplemented with $1 \mathrm{M}$ sorbitol; and resistance to ionic stress determined on YPD medium $+1 \mathrm{M} \mathrm{NaCl}$ agar. Sensitivity to metal ion stress was also tested on YPD medium agar supplemented with either $50 \mu \mathrm{M} \mathrm{CuCl}_{2}, 50 \mu \mathrm{M} \mathrm{ZnCl}_{2}$, or $10 \mathrm{mM} \mathrm{MnCl}$. Sensitivity to cell wall and membrane stress was compared on YPD medium agar supplemented with either $5 \mathrm{mM}$ caffeine, $100 \mu \mathrm{g} / \mathrm{ml}$ Congo red or $0.05 \%$ SDS. The ability to utilize non-fermentable carbon sources was also compared on YPG medium agar (YPD agar with 3\% glycerol in place of dextrose).

\section{RNA isolation and RTqPCR}

Each C. albicans strain was grown overnight in YPD medium at $30^{\circ} \mathrm{C}$ then subcultured to an $\mathrm{OD}_{600 \mathrm{~nm}}$ of 0.2 and then incubated at $30^{\circ} \mathrm{C}$ with shaking for 6 hours. Cells were pelleted by centrifugation before total cellular RNA was extracted using the hot phenol method [151]. cDNA was synthesized from total RNA using the Verso cDNA Synthesis Kit (Thermo Scientific) in accordance with the manufacturer's instructions. Synthesized cDNA was used for the amplification of ACT1 and the gene of interest by PCR, using SYBR green PCR master mix, according to the manufacturer's instructions. Gene-specific primers were designed using the PrimerQuest Tool from IDT, synthesized by Integrated DNA Technologies. The $2^{-\Delta \Delta} \mathrm{CT}$ method was used to calculate changes in gene expression among the strains [105]. All experiments included both biological and technical replicates in triplicate.

\section{Chemical libraries}

The NIH Clinical Collection (NCC) collection of 719 small molecules was provided by the NIH Small Molecule Repository. The Prestwick library of 1280 small molecules was purchased from Prestwick Chemical. The FDA Approved Oncolytic library (147 compounds) was provided by the NIH Developmental Products Program. 
Finally, the 10,000 compounds from the Myria collection were purchased from SigmaAldrich. All library compounds were supplied at $10 \mathrm{mM}$ in DMSO in 96-well plate format. These were diluted further in DMSO to final concentration of $1 \mathrm{mM}$, and $1 \mu \mathrm{l}$ volumes dispensed into round-bottomed 96-well plates that were used for the chemical screens.

\section{Chemical screening}

The FP tagged $C$. albicans strains expressing low, intermediate, and high levels of target protein were grown overnight in YPD medium at $30^{\circ} \mathrm{C}$, diluted to approximately 5 $\mathrm{X} 10^{3}$ cells $/ \mathrm{mL}$ in YNB medium, and mixed 1:1:1 to create the three-membered expression pools, or 1:1:1:1 for the four-membered pools. $199 \mu \mathrm{l}$ of the mixed cell suspension was then added to each well of each library plate, resulting in final compound concentration of $5 \mu \mathrm{M}$. After 24 and 48 hours incubation at $30^{\circ} \mathrm{C}, \mathrm{OD}_{600 \mathrm{~nm}}$ and fluorescence at the appropriate wavelengths were measured as described above. The relative fitness differential $(\mathrm{Rd})$ was then calculated for each well as the $\log _{10}\left[\mathrm{~T}_{\mathrm{Hi}} / \mathrm{T}_{\mathrm{Lo}}\right.$ fluorescence] and converted to Z-scores using the average and standard deviation of the $\mathrm{Rd}$ score for the whole collection. Hits were called based on a Z-score $\geq(+3)$ or $\leq(-3)$ in two independent replicates. Wells with little detectable $C$. albicans growth $\left(\mathrm{OD}_{600 \mathrm{~nm}} \leq\right.$ 0.1 ) were discarded from the analysis.

\section{Results}

\section{Design of target abundance-based fitness screening assay}

Strains expressing high $\left(\mathrm{T}_{\mathrm{Hi}}\right)$, low $\left(\mathrm{T}_{\mathrm{Lo}}\right)$, or intermediate $\left(\mathrm{T}_{\mathrm{Med}}\right)$ levels of the desired target protein are constructed, and each is labeled with a spectrally distinct FP tag. The tagged strains are then mixed together to form an expression pool that is used to screen and identify compounds that differentially affect the growth of each strain. The resulting chemically-induced population shift can be detected spectroscopically and indicates that a compound's activity depends upon the abundance of the target protein, therefore establishing a functional interaction between the chemical and target (Figure 3-1). As such, TAFiS depends upon two key technical challenges: (i) the capacity to label individual $C$. albicans strains with sufficiently bright and spectrally distinct FP tags and (ii) the stratification of target protein expression between $\mathrm{T}_{\mathrm{Hi}}, \mathrm{T}_{\mathrm{Med}}$, and $\mathrm{T}_{\mathrm{Lo}}$ strains.

\section{Selection and optimization of fluorescent protein tags}

The coding sequences of tagBFP, CER, GFP $\gamma$, YFP, $\varphi$ YFP, ZsYellow, dTOM, mCherry, and mPlum [142, 144-146, 152-155] were cloned into pKE4 which contains the powerful PTEF1, providing a constant high-level of expression. Each FP expression 


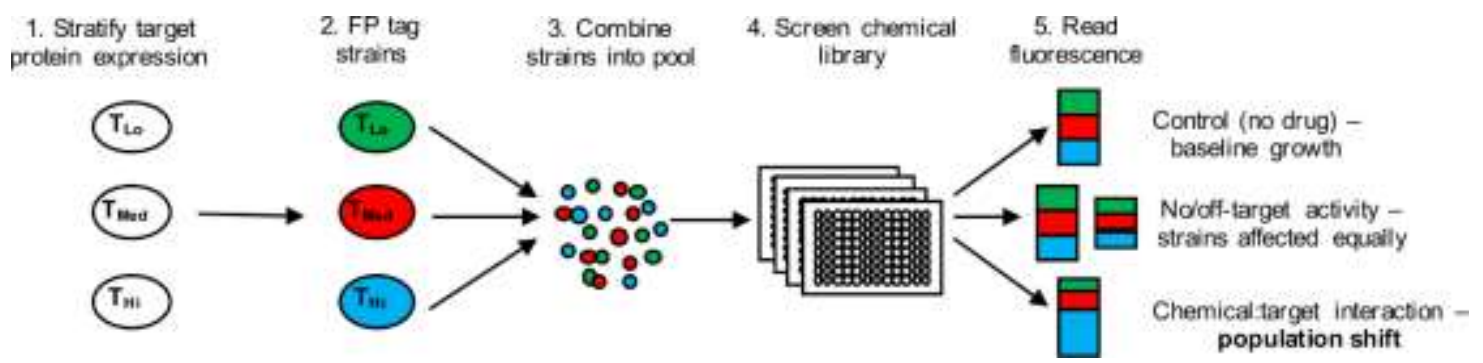

Figure 3-1. Schematic of the Target Abundance-based Fitness Screening (TAFiS) assay.

A panel of strains expressing high $\left(\mathrm{T}_{\mathbf{H i}}\right)$, medium $\left(\mathrm{T}_{\mathbf{M e d}}\right)$, or low $\left(\mathrm{T}_{\mathbf{L O}}\right)$ levels of the selected target are constructed, tagged with spectrally distinct fluorescent proteins (FP), and pooled. The expression pool is then incubated in the presence of various small molecules and the relative growth of each strain is quantified by measuring fluorescence. On-target inhibitors should differentially impact the growth of each strain causing a chemical-induced population shift that can be detected as a shift in fluorescence, as compared to the untreated control. 
construct was stably integrated into the genome of wild-type $C$. albicans strain. Then, 5 transformants of each expression construct were selected and seeded into 96-well plates in yeast nitrogen base (YNB) medium and then incubated at $30^{\circ} \mathrm{C}$ for 48 hours.

Fluorescence intensity was measured at the reported excitation and emission maxima of each FP and compared to a strain transformed with vector alone (untagged). Of those tested, the three FP tags with the highest signal to background under the assay conditions were cerulean (CER), green fluorescent protein gamma (GFP $\gamma$ ), and dTomato (dTOM) (Figure 3-2A). Next, we examined the degree of cross-excitation between these three FP tags by measuring the fluorescence signal of each tagged strain at each of the other FP wavelengths. Minimal spectral overlap was detected between CER-, GFP $\gamma-$, and dTOMtagged strains (Figure 3-2B), and these three FPs were therefore used in subsequent work. We next confirmed that the relative abundance of each tagged strain within a mixed population could be accurately quantified through fluorescence detection. Three wild-type $C$. albicans strains tagged with each FP were subcultured together in defined ratios and incubated in 96-well plates. After 24 and 48 hours, the fluorescence intensity of each FP tag was measured and plotted against the original inoculum. This produced a linear correlation with excellent $R^{2}$ values for each FP tagged strain (Figure 3-2C). Finally, we examined if high-level expression of CER, GFP $\gamma$, or dTOM was detrimental to $C$. albicans fitness by comparing the capacity of each tagged strain to endure a variety of stresses to that of an untagged control strain, including elevated temperature, osmotic and ionic stresses, as well as the presence of cell wall and membrane-perturbing agents. While the FP-tagged strains grew marginally slower than the untagged control strain under standard culture conditions (presumably as a result of the metabolic load of FP production), the effect was of similar magnitude irrespective of the FP tag expressed. The FP-tagged strains did not exhibit any additional abnormal phenotypes under any of the stress conditions tested (Figure 3-3). More recently, we have identified phi yellow fluorescent protein ( $\varphi$ YFP) as an exceptionally bright FP tag with limited overlap with the other FPs. $\varphi$ YFP also showed the same linear correlation, and phenotypes that the other FPs had shown.

\section{Altering DFR1 expression dramatically affects Candida albicans methotrexate susceptibility}

Dihydrofolate reductase (Dfr1p) is a historically important drug target for both antimicrobials and antineoplastic therapies [156-158] but has only recently been validated as an antifungal target (Chapter 2). Therefore we used Dfr1p to validate the TAFiS assay and test its reliability to detect on-target inhibitors (Figure 3-4A). We constructed two $D F R I$ expression pools, to test the performance of different techniques to alter the target expression. A DFRI overexpression strain (DFR1/DFR1/P ENOI-DFRI) was produced by introducing an additional copy of $D F R l$ into a wild-type (DFRI/DFRI) C. albicans strain, using an expression vector with the powerful $P_{E N O 1}$ transcriptional promoter. To suppress $D F R 1$ expression, a $d f r 1 \triangle / D F R 1$ heterozygous strain was constructed through the replacement of one DFRI allele with the HIS1 selection marker. Since we have shown that Dfr1p is an essential protein in $C$. albicans, it is not possible to construct a $d f r 1 \Delta / \triangle$ deletion mutant. Therefore, to further suppress $D F R l$ expression, a knockdown 

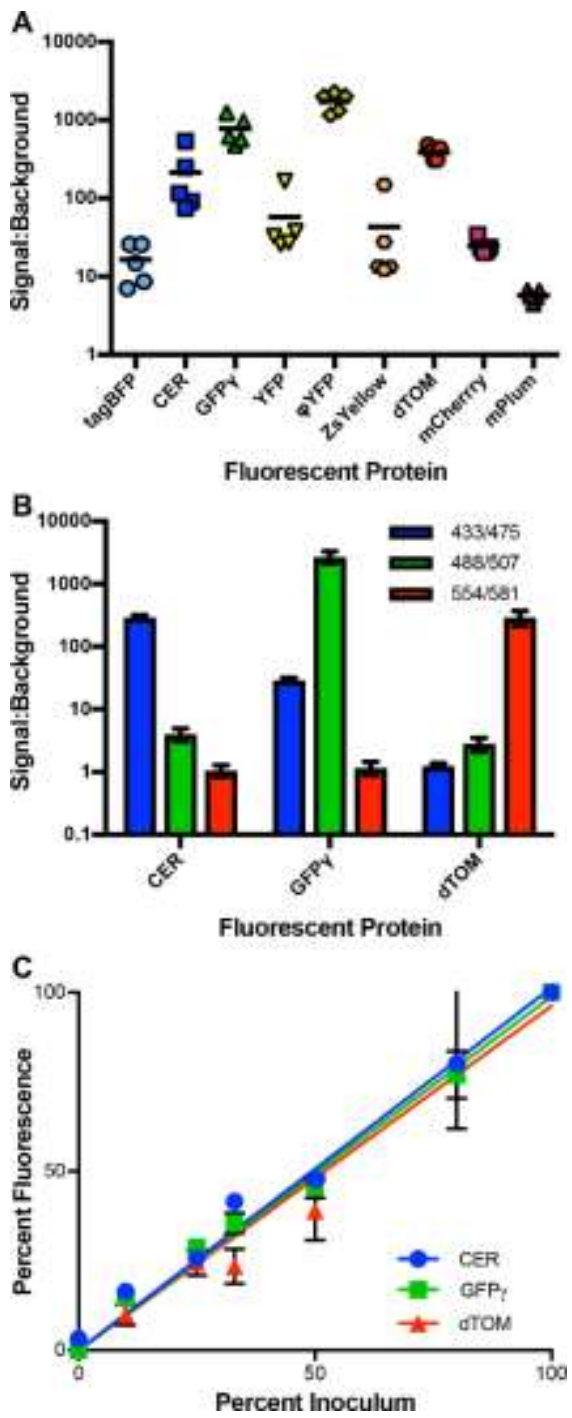

Figure 3-2. The relative percentage of each Candida albicans strain within a population can be accurately quantified by four spectrally distinct fluorescent proteins.

Fluorescence was read at the optimal excitation/emission wavelengths reported for five transformants with each FP. Brightness is expressed as fold-change over an untagged control strain. (B) The fluorescence of the brightest transformants expressing CER, GFP $\gamma, \varphi$ YFP, and dTOM was read at all four FP wavelengths (Ex./Em. 433/475, $488 / 507,529 / 550,560 / 590$ ) with a $9 \mathrm{~nm}$ bandpass to determine the extent of spectral overlap. The mean and standard deviations for each are presented. (C) The brightest strains expressing CER, GFP $\gamma, \varphi \mathrm{YFP}$, or dTOM were mixed in defined ratios and grown for 48 hours before fluorescence intensity was measured. Percent fluorescence at each FP's wavelength was then plotted against the percent of that tagged strain in the initial inoculum, resulting in linear correlations with excellent R-squared values for each tag. Values presented are the mean and standard deviations. 

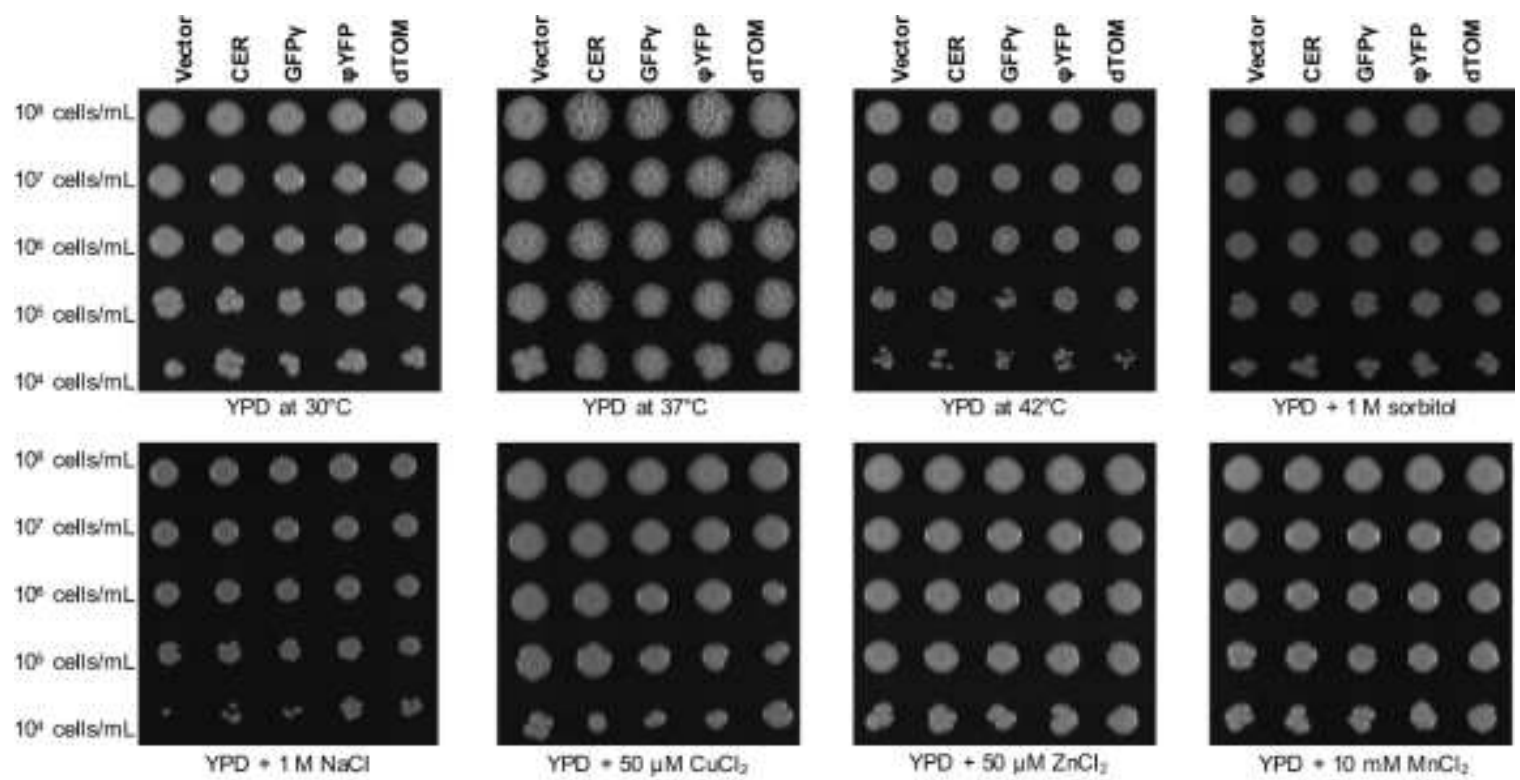

YPD $\approx 42 \mathrm{C}$

YFD $\rightarrow 1 \mathrm{M}$ sorbild
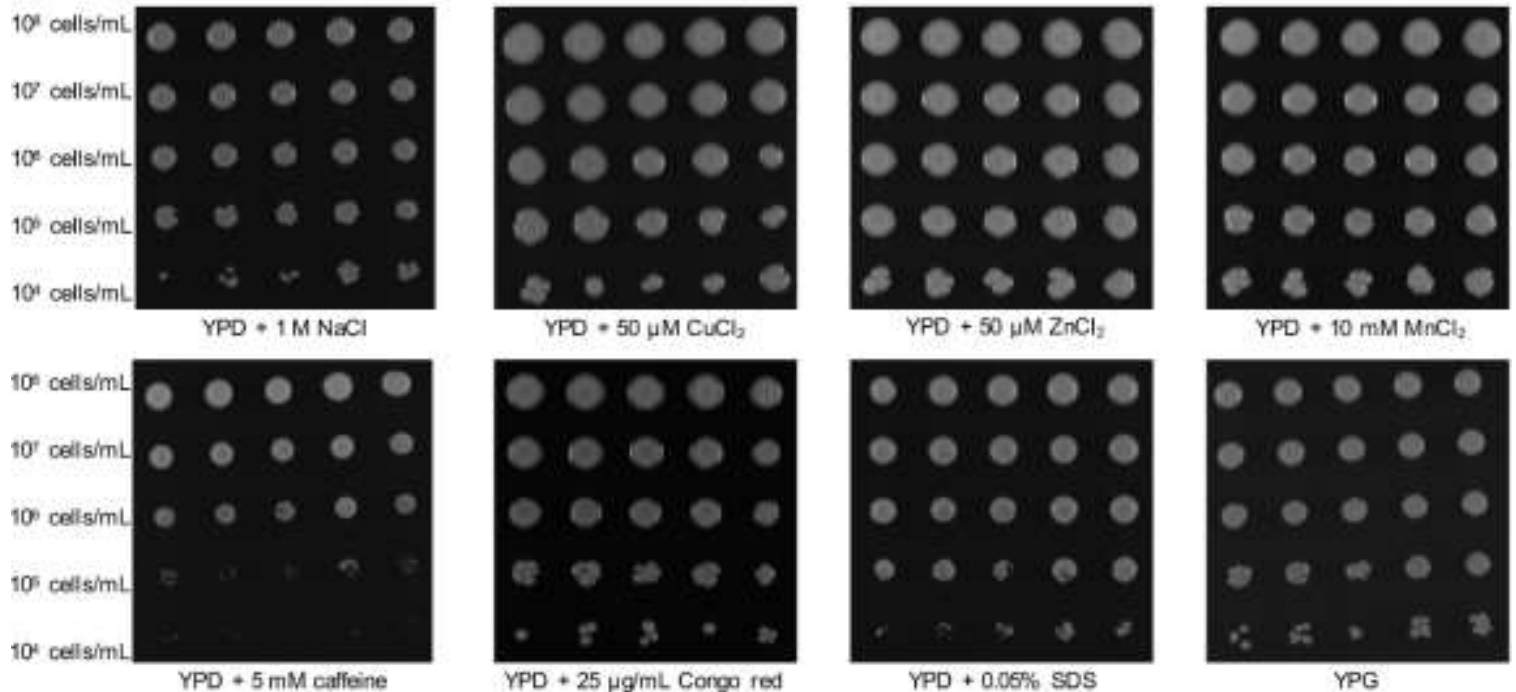

$\mathrm{YPD}+50 \mu \mathrm{M} \mathrm{ZnCl}_{2}$

$\mathrm{YPD}+10 \mathrm{mM} \mathrm{MnCl} 2$
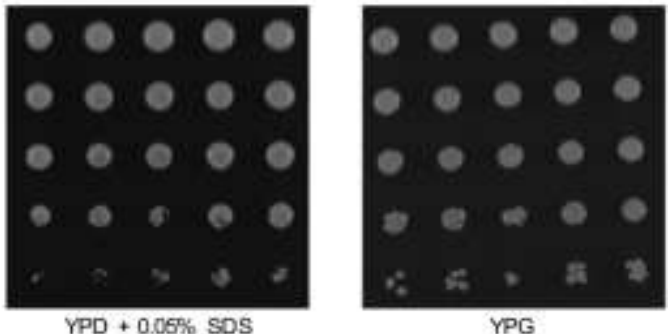

Figure 3-3. High level expression of the selected fluorescent proteins does not result in differential susceptibility to a variety of stress.

The brightest transformants expressing CER, GFP $\gamma, \varphi \mathrm{YFP}$ and dTOM were grown overnight in YPD at $30^{\circ} \mathrm{C}$. Cells were washed and resuspended in sterile water at approximately $1 \times 10^{7}$ cells $/ \mathrm{mL}$. Ten-fold serial dilutions were pinned on various media in duplicate and incubated for 48 hours at $30^{\circ} \mathrm{C}$, except when indicated otherwise, prior to imaging. Conditions tested were YPD, YPD at $37^{\circ} \mathrm{C}, \mathrm{YPD}$ at $42^{\circ} \mathrm{C}, \mathrm{YPD}+1 \mathrm{M}$ sorbitol, YPD + $1 \mathrm{M} \mathrm{NaCl}, \mathrm{YPD}+50 \mu \mathrm{M} \mathrm{CuCl}_{2}, \mathrm{YPD}+50 \mu \mathrm{M} \mathrm{ZnCl}_{2}, \mathrm{YPD}+10 \mathrm{mM}$ $\mathrm{MnCl}_{2}, \mathrm{YPD}+5 \mathrm{mM}$ caffeine, YPD + $25 \mu \mathrm{g} / \mathrm{mL}$ Congo red, YPD + $0.05 \%$ SDS, and YPG. 

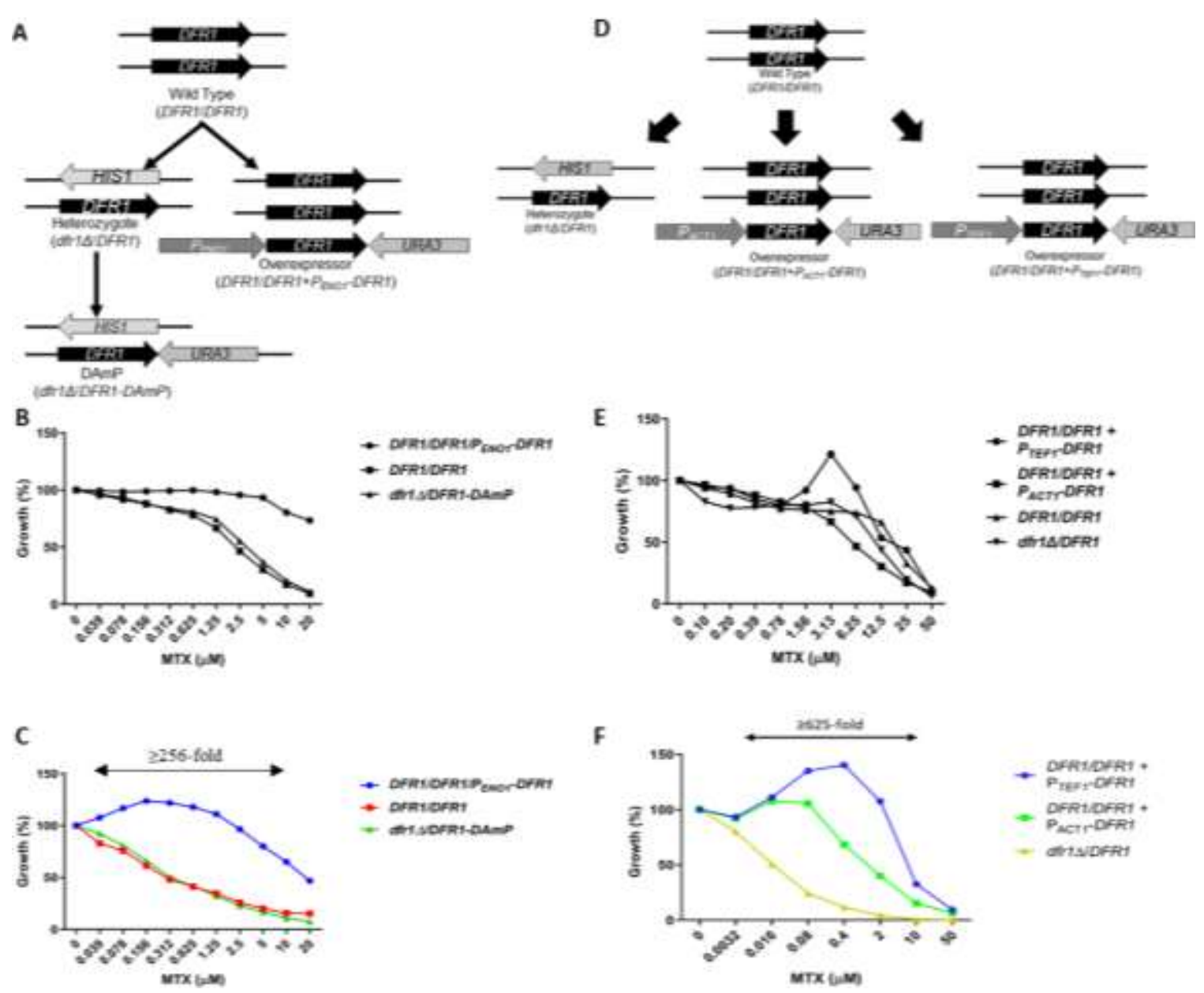

Figure 3-4. Modulating DFR1 expression affects Candida albicans susceptibility to methotrexate.

Schematic of strain construction of the first expression pool (A) or second expression pool (D). C. albicans strains were grown in YNB medium at $30^{\circ} \mathrm{C}$ in the presence of various concentrations of methotrexate for the first expression pool (B) or the second expression pool (E). After 48 hours, growth was measured via $\mathrm{OD}_{600 \mathrm{~m}}$ and expressed as a percentage of that measured in the untreated control. Values presented are the averages of technical triplicates. (C) C. albicans strains DFR1/DFRI/P ENOI-DFR1::CER, DFR1/DFR1::dTOM, and $d f r 1 \triangle / D F R 1-D A m P:: G F P \gamma$ or (D) DFR1/DFRI/P TEFIDFR 1::CER, DFR1/DFRI/PACTI-DFR1:: GFP $\gamma$, DFR1/DFR1::dTOM, and $d f r 1 \triangle / D F R 1:: \varphi$ YFP were combined to create an expression pool. Approximately $1 \times 10^{3}$ cells of the pool were inoculated into the wells of a 96-well plate in the presence of a range of methotrexate concentrations in YNB. After 48 hours at $30^{\circ} \mathrm{C}$, fluorescence was read at all three FPs' wavelengths and expressed as a percentage of fluorescence of the untreated wells. Values presented are the averages and standard deviations of technical triplicates. 
strain ( $d$ fr 1 $/$ /DFRl-DAmP) was produced using a technique known as decreased abundance by mRNA perturbation (DAmP) [150], to destabilize the mRNA transcript. RTqPCR confirmed a 30-fold differential in DFRI mRNA abundance between DFR I/DFR1/PENOI-DFR1 and $d f r 1 \triangle / D F R 1-D A m P$ strains. Next, we confirmed that changes in DFRI transcript altered $C$. albicans susceptibility to the on-target inhibitor methotrexate. Dose-response experiments confirmed that methotrexate (MTX) susceptibility differed by $\geq 8$-fold between DFRI/DFRI/PENOI-DFR1 and $d$ fr1 $1 / D F R 1$ DAmP strains (Figure 3-4B). A second $D F R I$ expression pool was constructed with an overexpression strain similar to the first but using the $P_{T E F 1}$ (DFR1/DFR1/PTEF1-DFR1), which drives higher levels of DFRI expression than the original $P_{E N O I}$. Then we constructed a third overexpression strain, with DFRI driven by the moderate $P_{A C T 1}$ (DFR1/DFR1/PACT1-DFR1). RTqPCR confirmed the expression differential to be $>100-$ fold between $D F R 1 / D F R 1 / P_{T E F 1-D F R l}$ and $d f r 1 \Delta / D F R 1$. Next, we confirmed that changes in Dfr1p abundance altered $C$. albicans susceptibility to the on-target inhibitor methotrexate. Dose-response experiments confirmed that methotrexate (MTX) susceptibility differed by $\geq 16$-fold between the highest- and lowest- DFRl-expressing strains (Figure 3-4E).

\section{Chemical-target interactions can be detected as spectroscopic shifts in the FP-tagged expression pools}

Each strain with altered $D F R l$ expression was each tagged with an FP expression construct. The DFR1/DFR1/PENO1-DFR1::CER, DFR1/DFR1::dTOM, and $d$ fr $14 / D F R 1$ DAmP::GFP $\gamma$ strains were then mixed together to form the first expression pool, and the DFR 1/DFR1/PTEF1-DFR1::CER, DFR1/DFR1/PACT1-DFR1::GFP $\gamma$, DFR1/DFR1::dTOM, and $d f r 1 \triangle / D F R 1:: \varphi Y F P$ were combined to form a second expression pool. Each pool was examined to determine how an on-target inhibitor affects the composition of the population. A total of approximately $1 \times 10^{3}$ cells from each $D F R 1$ expression pool, consisting of the three, or four, strains in equal proportions, were seeded to the wells of 96-well plates in YNB medium and grown for 48 hours in the presence of increasing concentrations of MTX. Fluorescence intensity was then quantified for each tag and compared to the minus-drug (dimethyl sulfoxide [DMSO]) control. As expected, a substantive population shift favoring the highest expression strain was detected in the expression pool over $\mathrm{a} \geq 256$-fold and $\mathrm{a} \geq 625$-fold concentration range of MTX concentrations compared to DMSO alone for the first and second DFRI pool respectively (Figure 3-4C and 3-4F). The same dose-response experiments were performed with a pool of three wild-type (DFRl/DFRl) strains (i.e., with no DFRl expression differential), tagged with CER, GFP $\gamma$, or dTOM, no significant spectral shifts were detected (data not shown). This confirmed that MTX induced a population shift in both DFRl expression pools as a result of differential DFRI expression, rather than interference with FP intensity. This result demonstrates that chemically-induced population shifts within an FP-tagged expression pool indicate a highly specific chemical-target interaction. To further determine the specificity with which TAFiS identifies chemical-probes, the DFRI strains for the first pool were subcultured together in equal proportions and incubated in increasing concentrations of known antifungal agents: fluconazole, caspofungin, and 
terbinafine (Figure 3-5). The intensity of each FP was then quantified and compared to the DMSO control. None of these off-target antifungals induced a population shift in the first DFRI pool, supporting that antifungals with MOAs unrelated to folate biosynthesis do not induce population shifts. Similarly, the Dfrlp strains were subcultured together in the presence of known antifolates that target another protein in the pathway, specifically dihydropteroate synthase. Neither sulfamethoxazole nor dapsone induced a population shift (Figure 3-5), further supporting that populations shifts in this expression pool indicates a target-specific chemical probe.

\section{TAFiS reliably identifies on-target inhibitors}

We next determined if TAFiS can provide the basis of a robust HTP chemical screening assay. The individual strains for each DFRl pool were grown to saturation and then mixed together in YNB medium to a final density of $2 \times 10^{5}$ cells $/ \mathrm{ml}$, in equal proportions to form the 2 separate DFRl pools. Each pool was then incubated in YNB medium in 96-well plates with alternating columns of $5 \mu \mathrm{M}$ MTX, or DMSO. To provide a single metric of comparative strain growth in each well, a relative fitness differential (Rd) was calculated as the $\log _{10}$ fluorescence intensity ratio of the highest $D F R I$ expression strain and lowest DFRI expression strain in each pool. Z -factors [159] were then calculated from the Rd scores of each DFRl expression pool incubated with or without MTX. Treatment of the first DFRI pool with $5 \mu \mathrm{M}$ MTX yielded a Z factor of $0.63 \pm 0.05$ while the second pool yielded a $Z$ factor of $0.65 \pm 0.09$, again supporting the excellent quality of the assay.

\section{TAFiS can provide an effective and efficient high-throughput chemical screen}

Finally, we tested the reliability of the TAFiS assay to identify on-target chemical probes when applied as a HTP-screen. For validation, the two separate DFR1 pools were used to screen the NIH clinical collection (NCC) of 719 compounds and the Prestwick library of 1280 compounds at a final concentration of $5 \mu \mathrm{M}$, each of which contains MTX. Wells with a total optical density at $600 \mathrm{~nm}(\mathrm{OD} 600 \mathrm{~nm})$ of $\leq 0.1$ were discarded from analysis as having populations insufficient to allow reliable detection of population shifts. Rd scores were calculated for each compound and converted to Z-scores based on the mean and standard deviation of the DMSO controls. Hits were called based on Zscores of $\geq(+3)$ or $\leq(-3)$ in both of two biological replicates. In the 3-membered pool, only MTX produced a positive Z-score $\geq(+3)$ (Note that amethopterin, also identified as a hit, is the same chemical entity). Unexpectedly, 21 compounds induced a statistically significant inverse population shift (Z-scores of $\leq[-3]$ ), indicating that the highest expression strain was more sensitive than the lowest expression strain (Table 3-2). Interestingly, many of the hits that produced negative Z-scores were azole antifungals or statins. This could be a result of the connection between the ergosterol pathway and the folate pathway, as the folate pathway supplies the methyl group used in the sterol C24 methyltransferase (Erg6p) [83]. 


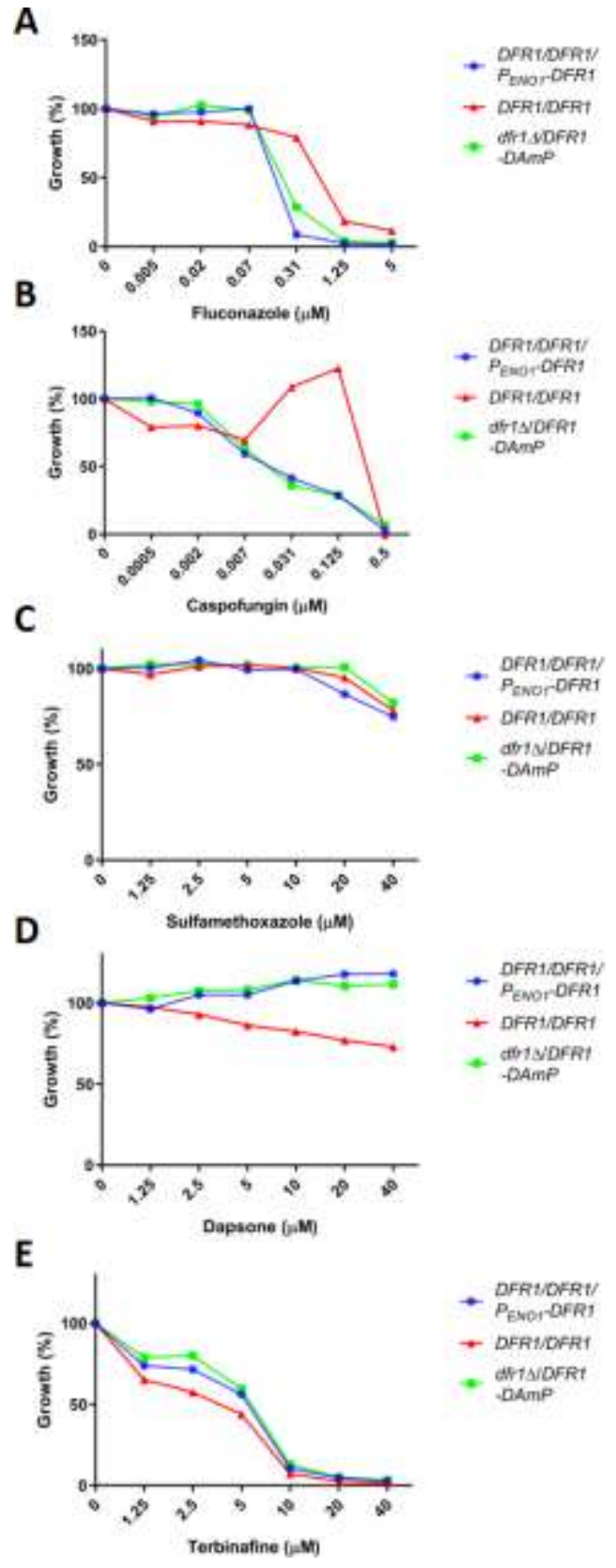

Figure 3-5. The first $D F R I$ expression pool only identifies compounds that specifically interact with the target protein.

C. albicans strains DFR1/DFR1/P ENO1-DFR1::CER, DFR1/DFR1::dTOM, and dfr1 $1 / D F R 1-D A m P:: G F P \gamma$ combined in equal proportions to create the first $D F R 1$ expression pool. Approximately $10^{3}$ cells of the combined pool were inoculated into YNB medium in the wells of a 96-well plate in the presence of a serial dilution series of (A) fluconazole, (B) caspofungin, (C) sulfamethoxazole, (D) dapsone, (E) terbinafine. After 48 hours at $30^{\circ} \mathrm{C}$, fluorescence was read at all three FP's wavelengths, and expressed as a percentage of fluorescence of the untreated wells. Values presented are the averages and standard deviations of technical triplicates $(\mathrm{N}=3)$ and are representative of two independent experiments. 
Table 3-2. Hit list from validation of TAFiS methodology using the first DFRI expression pool.

\begin{tabular}{|c|c|c|c|c|}
\hline Library & Concentration & Compound & $\begin{array}{c}\text { Mean Z- } \\
\text { Score }\end{array}$ & Structure \\
\hline \multirow[t]{11}{*}{$\mathrm{NCC}$} & $5 \mu \mathrm{M}$ & Methotrexate & 4.2 & \\
\hline & & Voriconazole & -3.4 & \\
\hline & & Miconazole & -3.6 & \\
\hline & & Triclabendazole & -3.8 & \\
\hline & & Disulfiram & -4.3 & \\
\hline & & Mupirocin & -4.7 & \\
\hline & & Fluconazole & -4.8 & \\
\hline & & Oligomycin A & -6.3 & \\
\hline & & Fluvastatin & -6.6 & \\
\hline & & Cerivastatin & -7.6 & \\
\hline & & Hexachlorophene & -8.0 & \\
\hline $\begin{array}{l}\text { Prestwi } \\
\mathrm{ck}\end{array}$ & $5 \mu \mathrm{M}$ & Amethopterin & 6.3 & \\
\hline
\end{tabular}


Table 3-2. (Continued).

\begin{tabular}{|c|c|c|c|c|}
\hline Library & Concentration & Compound & $\begin{array}{c}\text { Mean Z- } \\
\text { Score }\end{array}$ & Structure \\
\hline & & Methotrexate & 6.1 & \\
\hline & & Pyrvinium & -4.0 & \\
\hline & & Atorvastatin & -4.1 & \\
\hline & & Pemetrexed & -4.9 & \\
\hline & & Benzethonium & -5.6 & \\
\hline & & Haloprogin & -5.8 & \\
\hline & & Disulfiram & -5.9 & \\
\hline & & Chloroxine & -6.0 & \\
\hline & & Monensin & -7.6 & \\
\hline & & Merbromin & -9.1 & \\
\hline
\end{tabular}

Compounds that were identified when screening the first $D F R 1$ expression pool consisting of equal proportions of DFR1/DFRI/P ENOI-DFR1::CER, DFRI/DFR1::dTOM, and $d$ fr1 1 /DFR1-DAmP::GFP $\gamma$ cells against the NCC and Prestwick chemical libraries at $5 \mu \mathrm{M}$ along with their associated Z-scores for both biologically independent screens. 
The second expression pool identified MTX (and the same chemical matter amethopterin), raltitrexed, and silodosin as inducing statistically significant population shifts with positive Z-scores (Table 3-3). Interestingly, the second expression pool did not identify any statistically significant compounds with negative Z-scores. This reduced hit rate, especially of compounds causing negative Z-scores, could be a result of the increased expression differential or the addition of $\varphi$ YFP, both of which could make the pool more resilient to artifacts of the screen. However, both pools accurately identified the on-target inhibitor MTX as interacting with Dfr1p, which again supports that both expression pools reproducibly identify on-target chemical interactions.

\section{TAFiS readily identified novel compounds as potentially interacting with Dfr1p by screening diverse chemical matter}

To further validate our methodology and to identify novel chemical probes for Dfr1p, we screened a collection of 412 natural products, the FDA Approved Oncology library (129 compounds), and a selection of 10,000 compounds from the Myria collection using the second $D F R I$ pool. These screens were performed as before with each library compound added to a final concentration of $5 \mu \mathrm{M}$. Interestingly, the second DFRl pool identified no hits in the natural product library. In the Oncolytic library, MTX was identified as inducing a positive Z-score, and pemetrexed induced a negative Z-score. Of the 10,000 compounds from the Myria collection, 3 compounds were identified as causing statistically significant population shifts, 1 with a positive Z-score, and 2 with a negative Z-score (Table 3-2). Unfortunately, none of these hits were available for follow up experiments.

Finally, we repeated the screens with the Prestwick, FDA Approved Oncology, and Natural Products libraries in YNB without folate or PABA, to determine if the low concentration of folate in the YNB medium used in the original screens affected assay interference or hit identification. The second DFRl expression pool only identified MTX (and amethopterin) as statistically significant in these libraries under these conditions (Table 3-4), indicating that the presence of folate and PABA did not interfere with the identification of chemical-target interactions.

Initial follow up efforts focused on the 3 hit compounds with positive $\mathrm{Z}$ scores, MTX, raltitrexed, and silodosin. As already described, our positive control, MTX, induces a robust and dose-dependent population shift, however neither raltitrexed nor silodosin induced population shifts at any concentration tested, suggesting that these do not interact with Dfr1p (Figure 3-6). The identification of compounds that induced negative Z-scores was unexpected, as it implies that the low abundance strain has greater fitness than the high abundance strain. Dose-response experiments were performed with 10 of these compounds in an effort to confirm their activity. An inversely tagged $D F R 1$ pool, where $D F R 1 / D F R 1 / P_{T E F 1-D F R} 1$ expressed $\varphi$ YFP and $d f r 1 \triangle / D F R 1$ expressed CER, was utilized to identify compounds that interfered with fluorescent intensity or detection. Pooled dose-responses showed that merbromin induces dose-dependent fluorescent interference causing the same fluorescent shifts regardless of which strains expressed 
Table 3-3. Hit list from TAFiS validation using the second DFR1 expression pool.

\begin{tabular}{lll}
\hline Library & \multicolumn{1}{c}{ Compound } & Z-Score \\
Prestwick & Methotrexate & 27.5 \\
& Amethopterin & 25.2 \\
& Raltitrexed & 12.5 \\
& Silodosin & 7.9 \\
Oncolytic & Methotrexate & 11.4 \\
& $\begin{array}{l}\text { Pemetrexed } \\
\text { Myria }\end{array}$ & $\begin{array}{l}\text { 5-hydroxy-4-(2- } \\
\text { thienylcarbonyl)(1,2,5- } \\
\text { oxadiazol-3-yl) 2-thienyl } \\
\text { ketone } \\
\text { 2-(4,5-dibromo-6-hydroxy-3- } \\
\text { oxoxanthen-9-yl)benzoic acid } \\
\text { thio(2,2,4-trimethyl-4- } \\
\text { phenyl(6-1,2,3,4- } \\
\text { tetrahydroquinolyl))carbonitrile }\end{array}$ \\
\hline
\end{tabular}

Compounds that were identified when screening the second DFRl expression pool consisting of equal fractions of DFR1/DFR1/PTEFI-DFR1::CER, DFR1/DFR1/P ACT1- $_{\text {- }}$ $D F R 1:: \mathrm{GFP} \gamma, D F R 1 / D F R 1:: \mathrm{dTOM}$, and $d f r 14 / D F R 1:: \varphi$ YFP cells against the Prestwick, Oncolytic, and Myria chemical libraries at $5 \mu \mathrm{M}$ along with their associated Z-scores. 
Table 3-4. Hit list from TAFiS validation using the second DFR1 expression pool in the absence of folate and PABA.

\begin{tabular}{cccc}
\hline Library & Compound & Z-Score & Structure \\
\hline Prestwick & Methotrexate & 9.4 & \\
& Amethopterin & 9.1 & \\
Oncolytic & Methotrexate & 3.17 &
\end{tabular}

Compounds that were identified when screening the $D F R 1$ expression pool consisting of

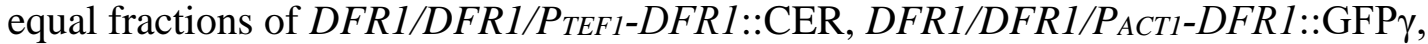
DFR1/DFR $1:: \mathrm{dTOM}$, and $d f \mathrm{r} 1 \triangle / D F R 1:: \varphi \mathrm{YFP}$ cells against the Prestwick, and Oncolytic chemical libraries at $5 \mu \mathrm{M}$ in the absence of folate and PABA along with their associated Z-scores. 
A

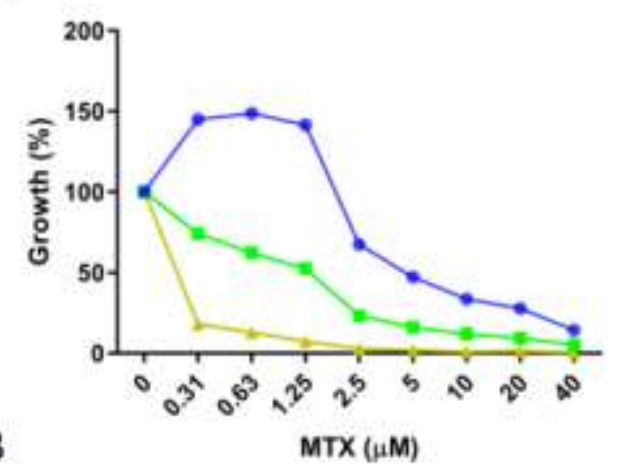

- $\begin{aligned} & \text { DFR1/DFR1/ } \\ & P_{\text {TEF } 1-D F R 1}\end{aligned}$

DFR1/DFR1/

- PACTI $_{\text {DFR1 }}$

- $\triangle$ DDFR1

B

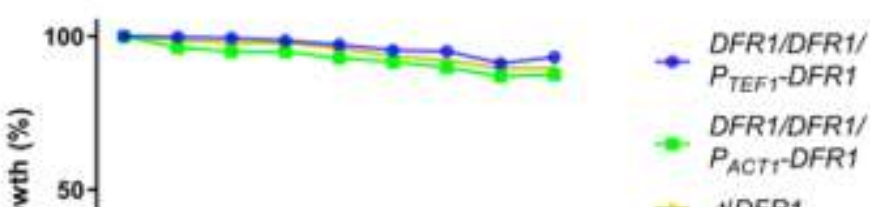

ธั่

- NDFR1

C

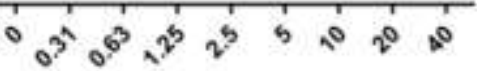

Raltitrexed $(\mu \mathrm{M})$

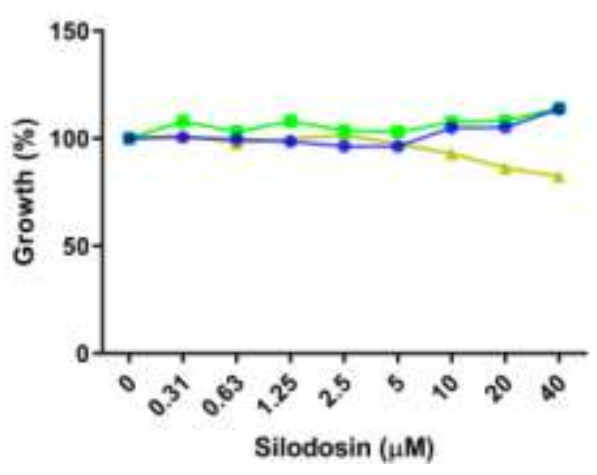

DFR1/DFR1/

- Pref -DFR1

DFR1/DFR1/

- $P_{A C T 1}$-DFR1

- NDFR1

Figure 3-6. The DFR1 expression pool identified methotrexate as interacting with Dfr1p.

Approximately $1 \times 10^{3}$ cells of the second $D F R 1$ expression pool were inoculated into the wells of a 96-well plate in the presence of a concentration range of (A) methotrexate, (B) raltitrexed, (C) silodosin in YNB. After 48 hours at $30^{\circ} \mathrm{C}$, growth was measured as OD $600 \mathrm{~nm}$ and is expressed as a percentage of untreated control. Values presented are the averages of technical duplicates and are representative of two independent experiments. 
which FP tags. In contrast, neither fluconazole, voriconazole, hexachlorophene, pyrvinium, disulfiram, haloprogin, monesin, fluvastatin, nor mupirocin induced population shifts in the second DFRI expression pool (Figure 3-7). The lack of reproducible population shifts indicates that these compounds do not interact with Dfr1p, and that the compounds that induced negative Z-scores are false positives.

\section{TAFiS is compatible with 384-well based high throughput screening formats}

In order to facilitate increased throughput and efficiency, we examined the performance of the TAFiS assay in 384-well plates using the second DFRI expression pool. Using volumes as low as $20 \mu \mathrm{l}$, the $C$. albicans strains tagged with CER, GFP $\gamma$, $\mathrm{dTOM}$, and $\varphi$ YFP demonstrated excellent signal/background ratios when compared to the untagged controls (data not shown). Furthermore, the second DFRI expression pool yielded Z factors of $0.71 \pm 0.03$ when treated with MTX at $5 \mu \mathrm{M}$, indicating excellent performance in the higher density format. Therefore, our approach can reliably identify target specific chemical probes in a variety of screening formats.

\section{Discussion}

The enormous costs and prolonged timelines associated with developing a new pharmacotherapy underscore the desperate need to increase the efficiency of both preclinical and clinical phases of drug discovery and development. Herein we describe a new type of TBWCS that can greatly expedite this process through the selection of physiologically-active and target-specific hits at the earliest possible stage, the primary screen. TBWCS have several major advantages over conventional target-based and cellbased screening strategies and, to a large extent, combines the benefits of both into a single assay. First, chemical probes are selected that functionally interact with a defined molecular target, providing invaluable insight into each hit's MOA. Second, the selected target is presented within its native environment, thus only compounds that can access and engage the target in its cellular context are recognized. Third, as the measured outcome is comparative growth of strains expressing different levels of target protein, TBWCS do not require purification of the target protein itself or a HTP-compatible biochemical assay of its activity in order to identify relevant chemical-target interactions. Indeed, this approach can readily be applied to targets that are not amenable to conventional biochemical assays, such as noncatalytic proteins. Fourth, compounds that lack specificity (e.g. that engage multiple targets) or which are generally toxic to cells are unlikely to create the target-dependent fitness differential upon which these approaches depend, and are thus eliminated at the primary screening stage.

Despite enormous potential advantages, TBWCS are underutilized as a drug discovery strategy. While several important studies in the pathogenic prokaryotes Staphylococcus aureus and Mycobacterium tuberculosis have collectively established the validity of TBWCS to discover on-target chemical probes [71-73], each has been restricted by several technical issues that have limited efficiency, sensitivity, and/or 
A
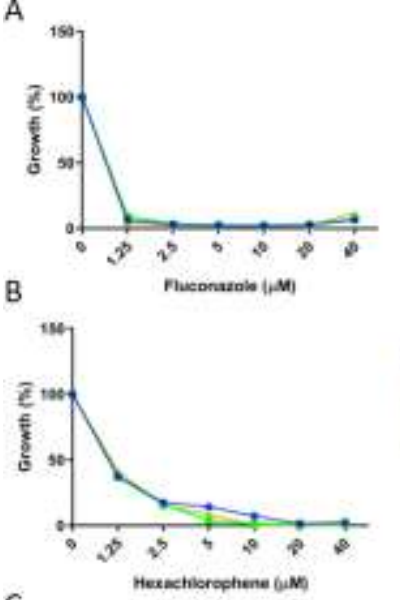

c
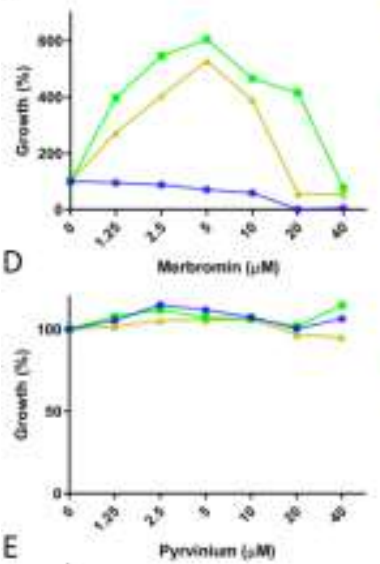

E

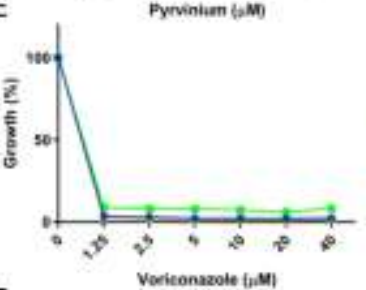

F

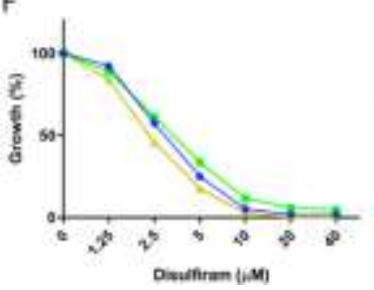

G

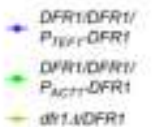

- ariafrer

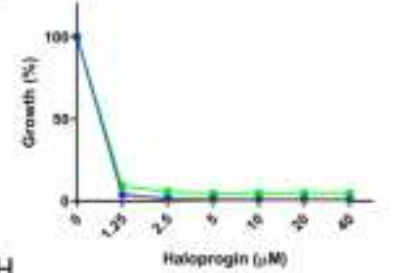

+ OPFricert:

- Prerofart

- Pafridafat

- mescer

H

- DFritofrN

afremeren

- netiadpat

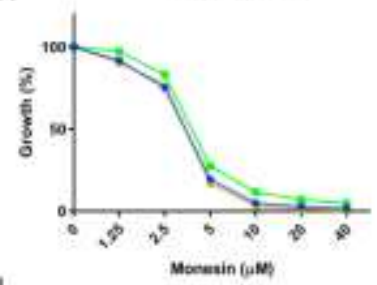

- DFFIDFAV

preiora.

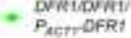

- arridefr

I

- orpmafry afrtofan

- DFRTIDERN

- ansome

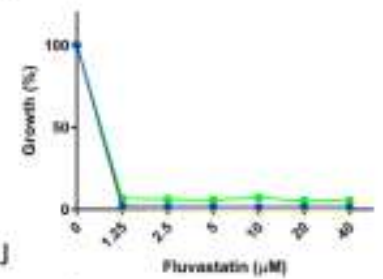

- DFPidafat

Derroviay

- PaetrofRt

- MTADFRt

J

- Dertopent

Pntrafer

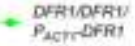

- artuarest

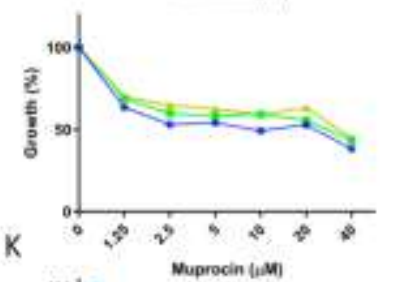

- DFRIORAS

DEATDFAY

- Pactroferl

4 shruarer

K

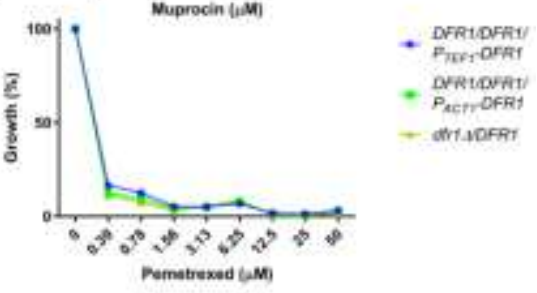

Figure 3-7. Compounds that induced statistically significant negative $Z$ scores do not induce population shifts.

Approximately $10^{3}$ cells of the DFRI expression pool were inoculated into the wells of a 96-well plate in the presence of a concentration range of (A) fluconazole, (B) hexachlorophene, (C) merbromin, (D) pyrvinium, (E) voriconazole, (F) disulfiram, (G) haloprogin, (H) monesin, (I) fluvastatin, (J) mupirocin, and (K) pemetrexed in YNB. After 48 hours at $30^{\circ} \mathrm{C}$, growth was measured as $\mathrm{OD}_{600 \mathrm{~nm}}$ and is expressed as a percentage of untreated control. Values presented are the averages of technical duplicates and are representative of two independent experiments. 
throughput of the screens. Our approach builds upon previous efforts but also incorporates several technological advances that improve sensitivity and efficiency. First, previous TBWCS compared only the growth of a knockdown strain, with reduced target expression, to a reference strain expressing normal levels of the target protein. In our approach, the effect of test compounds on both knockdown and overexpression strains can be simultaneously compared to the effect on a reference strain. This improvement should enhance both the dynamic range and sensitivity of the screening assay to detect functional chemical-target interactions. Second, previous TBWCS invariably used twoplate assays that compare the growth of the reference and knockdown strains on separate culture plates. With TAFiS, the overexpression, reference, and knockdown strains are subcultured together, and thus the relative growth of each strain is compared in a single well to improve screening efficiency. Third, previous TBWCS were based on measurements such as colony size or zones of growth inhibition. These outcomes are not suited for quantitative measurement, automated data collection, or statistical analysis. Through the incorporation of fluorescent protein tags, TAFiS facilitates straightforward, quantitative measurements of the relative growth of each strain, providing an outcome that is readily amenable to statistical analysis. This simplifies the selection, ranking, and prioritization of hits according to the magnitude of the observed population shift and by inference the strength of the compound-target interaction.

Finally, in addition to identifying compounds that directly engage the selected target protein, TBWCS have the potential to identify compounds that indirectly interact with the selected target protein. For instance, when the abundance of the target protein is limiting, a compound that interacts with a compensatory/redundant pathway may preferentially inhibit the knockdown strain's growth. Compounds with indirect interactions are potentially important as they may uncover new functional interactions between distinct cellular pathways or enhance the efficacy of on-target drugs and thus provide a basis for combination therapy. However, our studies with Dfr1p indicate that TAFiS is exceptionally reliable at identifying chemical probes that directly interact with the target. Only 3 hits were identified as causing positive population shifts in the screens that were not confirmed in follow up assays. Intriguingly, several compounds were identified that resulted in negative $\mathrm{Z}$ scores, indicating that the highest expression strain is more susceptible to these agents than the lowest expression strain. While the exact meaning of these interactions is unclear, none of the negative hits that we tested induced population shifts in follow up assays, indicating that negative Z-scores.

One potential concern with TAFiS is that small molecules may interact with or selectively quench the fluorescent protein tags, resulting in false positives. However, this was not a major problem in our assay, which identified very few compounds that interfered with fluorescent measurement. This is likely because our assay used a selection of closely related fluorescent proteins, derived from the original GFP (e.g., CER, GFP $\gamma$, and $\varphi$ YFP). This selection should ensure that unique fluorescent protein interactions are a rare occurrence, as the related FP tags would respond similarly. Nonetheless, such false positives can be rapidly eliminated using a simple counterscreen to test the effect of each hit on an inversely FP-tagged pool of strains or a pool of wild-type strains tagged with the FPs. Chemicals that induce the same spectral shifts in this inverse pool, as in the original 
pool, interfere with the fluorescent signal or detection and should be eliminated from further analysis. Finally, the field of fluorescent protein engineering [160, 161], as well as the instruments used for their detection, is rapidly evolving. We anticipate that as brighter FPs with more defined spectral profiles become available, there will be the potential to simultaneously screen multiple expression pools representing multiple target proteins within a single well. Such multiplexing may facilitate the simultaneous screening against all components of a pathway and will yield further cost and time savings.

We constructed two different expression pools for DFRl to investigate the performance of different techniques. The 4-membered pool had increased expression and susceptibility differentials compared to the 3-membered pool. Both pools were able to identify the known on-target inhibitor MTX in chemical screens, but the 3-membered pool identified no other hits with positive Z-scores, while the 4-membered pool identified raltitrexed and silodosin, neither of which were confirmed in follow up dose-responses. Interestingly, the 3-membered pool identified many hits with negative Z-scores in a single library, while the 4-membered pool only identified 2 across several libraries. The false positive rate was not consistent between the two pools, which could indicate that pool specific features, such as the expression differential and the FP tags chosen, influence the false hit rate. The hits with negative Z-scores could be a result of the FPs chosen, but most of these hits did not appear to interact with the FP intensity.

Alternatively, the hits could be a result of the differences in strain construction, since the 3-membered pool identified more than the 4-membered pool. However, none of the hits with negative Z-scores were confirmed in follow up dose responses. Therefore, both expression pools confirm that TAFiS reliably identifies on-target inhibitors, validating the approach.

In addition to Dfr1p, we utilized the well-characterized target protein lanosterol demethylase (Erg11p) to validate this approach. While expression pools for both targets reliably identified known on-target inhibitors, there were differences between the two expression pools. First, the Erg11p pool treated with the on-target inhibitor fluconazole yielded consistently higher Z -factors than the DFR 1 pool treated with MTX; $0.86 \pm 0.04$ in 96 well plates versus $0.65 \pm 0.09$, and $0.78 \pm 0.03$ in 384-well plates compared to 0.71 \pm 0.03 for the second $D F R 1$ pool. Pools for both targets specifically and reproducibly identified the expected on-target inhibitors, the Erg11p expression pool identified and confirmed 2 new compounds that interacted with Erg11p at sub-growth inhibitory concentrations and were previously unknown to interact with the target. Both diethylstilbestrol, and hexestrol were identified as causing significant positive Z-scores in the Prestwick library, and were confirmed in follow up dose-responses. In addition, when wild-type $C$. albicans strains were treated with diethylstilbestrol or hexestrol, sterol profiling was consistent with inhibition of Erg11p. Unfortunately, no such new interactions were identified using either DFRI expression pools in the libraries utilized. Both pools demonstrated the ability to specifically and reliably identify compounds known to interact with the target of interest.

Moving forward, when applying TAFiS to additional systems, it is crucial to remember that there are a variety of both target- and compound-specific factors that may 
influence both the magnitude and direction of the observed population shifts. Targetspecific factors may include the magnitude of the target expression differential, the consequences of hypo- and hyperactivity of the target protein, and the subunit composition of the target. Maximizing the target protein expression differential between the strains should increase the range of concentrations over which an on-target probe differentially impacts the fitness of the strains. For an essential protein, this is limited by the minimal target protein activity that is sufficient to sustain cell viability. However, for a nonessential protein, a gene deletion strain can be incorporated into the expression pool. Accordingly, the population shift induced by a direct chemical-target interaction would be different, as a target deletion strain should be insensitive to target specific interactions. Chemical-specific factors that may influence outcomes in the TAFiS assay include the strength of the chemical-target interaction, the precise mode of action (e.g., inhibition versus activation, competitive versus non-competitive, active site versus allosteric), and the specificity of target engagement. Finally, TAFiS is theoretically applicable to any microbial species that is culturable and genetically tractable, the scope of which was expanded substantially with the development of CRISPR based systems. We anticipate the development of closely related methodologies in a variety of other microbes, including bacterial, protozoan, and fungal pathogens. Due to the ease of recombinant protein expression in the yeast Saccharomyces cerevisiae, adaptation of TAFiS to this system could facilitate chemical screens using targets derived from nonculturable microbes, species not amenable to genetic manipulation, or even disease-relevant human proteins. 


\section{CHAPTER 4. IDENTIFICATION AND STRUCTURE-ACTIVITY RELATIONSHIPS OF INHIBITORS OF FUNGAL FATTY ACID BIOSYNTHESIS}

\section{Introduction}

Invasive fungal infections (IFIs) are estimated to cause 1.5 million deaths annually [1]. Candida albicans accounts for 400,000 of these annually, with a mortality rate of around $40 \%[1,7]$. Millions more suffer from debilitating mucosal infections or subcutaneous mycoses $[2,3]$. More concerning is that the population of susceptible individuals continues to rise with the increasing use of broad-spectrum antibiotics, invasive surgeries, indwelling catheters, and immunosuppressive therapies [12-15]. The high rates of mortality for patients with IFIs are in part due to the limitations of each class of systemic antifungal drugs that include patient toxicity, narrow spectrum of activity, and/or the emergence of fungal resistance $[53,162]$. More generally, the clinical efficacy of currently approved antifungals is also disappointing. Indeed, up to $46 \%$ of patients with disseminated candidiasis will not respond well to fluconazole or other azoles [42, 43, 45-48, 61]. Approximately $28 \%$ of patients fail therapy with Amphotericin B [54, 55], which is the only polyene antifungal approved for systemic use. The most recent antifungal class, the echinocandins, is only formulated for intravenous administration, has a narrow-spectrum of activity, and up to $48 \%$ of patients fail therapy on caspofungin [44, $48,54,55,60]$. As such, there is an urgent need to develop new antifungal drugs with novel mechanisms of action to overcome the growing problem of antifungal resistance and improve patient outcomes.

Fatty acids (FA) are a defining constituent of biological membranes and are therefore essential for life. They also provide important energy stores and posttranslational modifications crucial for attachment and the function of many membranebound proteins [163]. FA synthesis involves repetitive cycles of a seven-step sequence of reactions that transfer 2 carbon units from malonyl-CoA to extend a hydrocarbon chain $[163,164]$. FA desaturase enzymes subsequently introduce double bonds into the hydrocarbon chain to yield unsaturated fatty acids (UFAs), which are crucial for regulating membrane fluidity.

While the FA biosynthetic enzymes of prokaryotes are usually monomeric [165], they form large multimeric complexes in eukaryotes [166]. These structural differences have been exploited to derive antibacterial agents, including triclosan and isoniazid that target the enoyl acyl carrier protein reductase [86, 87]. Recent studies have also revealed notable differences in the structure of the fungal and mammalian fatty acid synthase (FAS) complexes [164, 166]. The latter comprises a homodimer of $\sim 540 \mathrm{kDa}$ [167] with two independent reaction chambers arranged in a flexible X-shaped configuration [164]. In contrast, fungal FAS is a massive $2.6 \mathrm{MDa}$ heterododecameric complex consisting of 6 $\alpha$ (Fas2p) and $6 \beta$ (Fas1p) subunits that form a rigid barrel-shaped structure with two reaction chambers that contain three copies of each domain $[164,166]$. The biochemical mechanism of the fungal FAS starts as an acetyl-CoA is loaded onto the acetyl- 
transferase, located on Fas1p. The acetyl-CoA is shuttled to Fas2p for the 3-ketoacyl synthase where malonyl-CoA is combined with the acetyl-CoA. The molecule is then moved to the 3-ketoacyl reductase on Fas2p, where the carbonyl is reduced to an alcohol. Then the growing carbon chain is moved to the enoyl dehydratase on Fas $1 p$, where the alcohol group is removed to form a double bond between the $2^{\text {nd }}$ and $3^{\text {rd }}$ carbon from the acyl carrier protein. This double bond is then reduced by the enoyl reductase on Fas1p, to form a saturated carbon chain. This sequence is repeated, adding two carbon units until the chain reaches a 14 or 16 carbon length. This complex is followed by Ole1p, a stearoyl-CoA $\Delta 9$ desaturase which produces monounsaturated fatty acids [168]. Both FAS and the Ole1p desaturase enzymes are essential for fungal viability [88, 168, 169]. Exogenous supplements of appropriate FAs can partially restore the growth of FAS and Ole1p deficient mutants in vitro; however, these enzymes are essential for infection within the mammalian host [88]. C. albicans, Candida parapsilosis, and Cryptococcus neoformans FAS deficient mutants are all unable to colonize tissue in a mouse model of disseminated infection, highlighting that FA biosynthesis is essential in vivo $[88,170$, 171]. In addition, suppression of FAS or Ole1p expression using a doxycyclinerepressible system was sufficient to clear $C$. albicans from the tissues of mice with established disseminated infections [88]. Thus, both FAS and Ole1p can provide potentially efficacious targets for antifungal intervention. However, attempts to identify inhibitors of these enzymes are inherently difficult. Ole1p is an integral membrane protein and the membrane is essential for the integrity and function of the enzyme. The colossal size and multi-subunit architecture of the FAS complex makes purification of the functional enzyme impractical [172-175]. As such, it has not been possible to devise high-throughput compatible biochemical assays that can be used for target-based chemical screens. However, inhibitors of proteins in the fungal FA pathway have been reported. Becker and colleagues identified several compounds sharing a 1,2,4triazolidine-3-thione core with antifungal activity against $C$. albicans and applied a chemical-genetic approach to infer the target as FA desaturase [88, 176]. This mechanism of action was further supported by the restoration of fungal growth through the addition of exogenous UFAs and biochemical data demonstrating these compounds reduced the UFA content of $C$. albicans cells. Mor and colleagues identified 2 hydrazide containing compounds that inhibited sphingolipid biosynthesis in Cryptococcus neoformans but had poor activity on $C$. albicans [177].

The purpose of this study is to utilize a whole-cell approach to identify inhibitors of $C$. albicans FAS and Ole1p. This was achieved by using the target abundance-based fitness screening (TAFiS) methodology which we previously validated using DHFR. 


\section{Materials and Methods}

\section{Growth conditions}

C. albicans was routinely grown on yeast extract-peptone-dextrose (YPD) agar plates at $30^{\circ} \mathrm{C}$, supplemented with $50 \mu \mathrm{g} / \mathrm{ml}$ uridine for $u \mathrm{ra} 3 \Delta / \Delta$ strains. Selection of $C$. albicans transformants was carried out on minimal YNB medium $(6.75 \mathrm{~g} /$ liter yeast nitrogen base without amino acids, $2 \%$ dextrose, $2 \%$ Bacto agar) supplemented with the appropriate auxotrophic requirements described for S. cerevisiae [178] or with $50 \mu \mathrm{g} / \mathrm{ml}$ uridine.

\section{Plasmid construction}

The $C$. albicans expression vector pKE4 has been previously described [104]. pKE4: FAS1 was generated by PCR amplifying the FAS1 ORF from SC5314 using the primer pair FAS1ORFF-Sal1 and FAS1ORFR-Mlu1 and cloning the resulting product between the Sal I and Mlu I sites of pKE4. pKE4: OLE1 was generated by PCR amplifying the OLE1 ORF from SC5314 using the primer pair OLE1ORFF-Sal1 and OLE1ORFR-Mlu1 and cloning the resulting product between the Sal I and Mlu I sites of pKE4. All plasmid constructs were sequence verified to ensure the correct cloning of products and the absence of unintended mutations.

\section{Candida albicans strain construction}

BWP17 [103] was kindly provided by Aaron Mitchell (Carnegie Mellon University). C. albicans was transformed with DNA constructs using the lithium acetate procedure [100]. Gene deletion strains were constructed using the PCR-based approach described by Wilson et al. [103]. All pKE4-based vectors (URA3 selection marker) were cut with Nhe I prior to the transformation of ura3 $\Delta / \Delta$ recipient $C$. albicans strains to target integration at and fully restore the URA3-IROI locus. All pGHT-based vectors (HIS1 selection marker) were cut with Nru I prior to the transformation of his 14/4 recipient $C$. albicans strains to target integration at and fully restore the HISI locus.

The Ole1p and FAS strains were made using the same methods. The FAS1 gene deletion cassette was amplified using primer set FAS1DISF and FAS1DISR and plasmid pRSARG4 $\triangle$ SpeI (ARG4 selection marker) as a template and transformed into BWP17. Correct integration of the gene deletion cassette to replace one FAS1 allele was confirmed by diagnostic PCR, using primers ARG4INTR2 and FAS1AMPR-SacI as well as ARG4INTF2 and FAS1AMPF-KpnI. The promoter replacement strain was made by amplifying the promoters of various genes from previously published plasmids [104] using FAS1PRF and FAS1PRR, and these promoter replacement cassettes were transformed into the FASl heterozygote. Oligonucleotides used in this study are included in Table 4-1. 
Table 4-1. Oligonucleotides used in Chapter 4.

\begin{tabular}{|c|c|}
\hline Primer & Sequence \\
\hline FAS1DISF & $\begin{array}{l}\text { TGAGGTATAAACTTTCCCCCCTATTTTAGCCTATAATTATTTATATCC } \\
\text { TGTTTCCATATTACACAGTTTTCCCAGTCACGACGTT }\end{array}$ \\
\hline FAS1DISR & $\begin{array}{l}\text { AGGAATATATTTACCAATCAAATCTTGAGGCTTGACGGACGATTTTG } \\
\text { GAATCTTCTTGCACAAGATGTGGAATTGTGAGCGGATA }\end{array}$ \\
\hline FAS1AMPF & TGTGTAGATTCAATACAGTCG \\
\hline FAS1AMPR & AGACCCATCGAGCCCAACTGG \\
\hline ARG4INTR2 & AATGACTGAATTATGTCGGTC \\
\hline ARG4INTF2 & AAGCTAGTGTGGAAAGAAGAG \\
\hline FAS1PRF & $\begin{array}{l}\text { CCAACATTTAAAAAACTGTTGGGACTAACCTGCATAGTGAGGGAGG } \\
\text { TCCTGTAGATAAACAACAAAGTTGAACTCCCTTATGGTGC }\end{array}$ \\
\hline FAS1PRR & $\begin{array}{l}\text { TTTGGTACCAATAAGGTATGTTCAATTGAACCATGAGTTAATTGGAA } \\
\text { AGGTCTATGAGTTGACATACGTCGCATGCTCCCGGCCG }\end{array}$ \\
\hline $\begin{array}{l}\text { FAS1ORFF- } \\
\text { SalI }\end{array}$ & TCAGTCGACATGTCAACTCATAGACCTTTCC \\
\hline $\begin{array}{l}\text { FAS1ORFR- } \\
\text { MluI }\end{array}$ & TCAACGCGTACAGGTCCTTTAAATAGCAAG \\
\hline FAS1qF2 & CAGGTGGTGTTTCTGGA \\
\hline FAS1qR2 & TACTCATCGTCAATAGGG \\
\hline OLE1DISF & $\begin{array}{l}\text { CAAGTAACACATACTGTTATTACAAGAACTTGAAAGTCAAGACCAA } \\
\text { AACAAATTAGCATTTCACAGTTTTCCCAGTCACGACGTT }\end{array}$ \\
\hline OLE1DISR & $\begin{array}{l}\text { ATGACAGAAAATGAAAACGAAAAATGAAAGAAAACAAATCAACCC } \\
\text { AGCAATTTTCTATTTGCCTATGTGGAATTGTGAGCGGATA }\end{array}$ \\
\hline $\begin{array}{l}\text { OLE1AMPF- } \\
\text { SacI }\end{array}$ & TCAGAGCTCTCAGCATTAACTTTGGTTGGC \\
\hline $\begin{array}{l}\text { OLE1AMPR- } \\
\text { SacI }\end{array}$ & TCAGAGCTCCTGGACCAGACCACCTTTTGG \\
\hline OLE1PRF & $\begin{array}{l}\text { AAAGGGGACTAAAATAACAGACATCTACTAGTGAGAATTTCATAAA } \\
\text { TTAAGAAATATTCAAGAGAAGTTGAACTCCCTTATGGTGC }\end{array}$ \\
\hline OLE1PRR & $\begin{array}{l}\text { GTACCAGCAGCAATGGCATTCAATTTAGTGATATCAACAGTTTCAAG } \\
\text { TTGTTCAACTGTAGTCATACGTCGCATGCTCCCGGCCG }\end{array}$ \\
\hline $\begin{array}{l}\text { OLE1ORFF- } \\
\text { SalI }\end{array}$ & TCAGTCGACATGACTACAGTTGAACAACTTG \\
\hline $\begin{array}{l}\text { OLE1ORFR- } \\
\text { MluI }\end{array}$ & TCAACGCGTACCCAGCAATTTTCTATTTGC \\
\hline OLE1qF2 & GGTCATTCTCATCGTATTC \\
\hline OLE1qR2 & CTTTGGGTTGGCTTTAG \\
\hline
\end{tabular}




\section{Antifungal susceptibility testing}

Stock solutions of Cerulenin (Sigma) and ECC190 (UTHSC Medicinal Chemistry Core) were prepared at $10 \mathrm{mM}$ in DMSO and diluted as needed in the same solvent. Each C. albicans strain was grown overnight in YPD at $30^{\circ} \mathrm{C}$ and diluted to $1 \times 10^{4}$ cells $/ \mathrm{ml}$ in YNB medium, and $100 \mu \mathrm{l}$ of each cell suspension was transferred to the wells of a roundbottom 96-well plate. An additional $100 \mu$ of YNB medium containing $2 x$ the final desired concentration of each drug was then added to each well. The final concentration of DMSO was $0.5 \%$ for all treatments. Plates were then incubated at $30^{\circ} \mathrm{C}$ before growth was quantified after 24 and 48 hours by measuring $\mathrm{OD}_{600 \mathrm{~nm}}$ using a Cytation 5 plate reader (Bio-Tek Instruments, Inc.). The growth of each strain at each drug concentration was then expressed relative to the DMSO-alone control. The multistrain competitionbased experiments were performed as described above, except the individual FP labeled strains were diluted to $1 \times 10^{4}$ cells $/ \mathrm{ml}$, mixed 1:1:1:1 to create the four-member expression pools before they were dispensed into the 96-well, or 384-well, plates. Following incubation at $30^{\circ} \mathrm{C}$ for 48 hours, OD $600 \mathrm{~nm}$ and fluorescence intensity at the four wavelength pairs CER (ex 433/ em 475), GFP (ex 488/ em 507), dTOM (ex 560/ em 590), and $\varphi$ YFP (ex 529/ em 550) were measured as described above. The relative growth of each strain in the presence of drug was then expressed as a percentage of the same strain's growth in the minus-drug (DMSO-alone) control pool, as measured by the fluorescence intensity of the corresponding FP label.

\section{RNA isolation and RTqPCR}

RNA was isolated using the hot phenol method of RNA isolation described previously [104]. This RNA pellet was washed with $500 \mu \mathrm{l}$ of $70 \%$ ice-cold ethanol and collected by centrifugation. The RNA pellet was resuspended in DNase/RNase-free $\mathrm{H}_{2} \mathrm{O}$. cDNA was synthesized from total RNA using the Verso cDNA Synthesis Kit (Thermo Scientific) in accordance with the manufacturer's instructions. Synthesized cDNA was used for both the amplification of $A C T 1$ and the gene of interest by PCR, using SYBR green PCR master mix, according to the manufacturer's instructions. Genespecific primers were designed using the PrimerQuest Tool from Integrated DNA Technologies (IDT), and synthesized by IDT. The PCR conditions consisted of an initial denaturation at $95^{\circ} \mathrm{C}$ for 10 minutes, followed by 40 cycles of denaturation $\left(95^{\circ} \mathrm{C}\right.$ for 15 seconds), annealing $\left(60^{\circ} \mathrm{C}\right.$ for 30 seconds), and extension $\left(72^{\circ} \mathrm{C}\right.$ for 37 seconds).

Software for the 7500 System Sequence Detection Software version 1.2.3 from Applied Biosystems was used to determine the dissociation curve and threshold cycle (CT). The $2^{-\Delta \Delta} \mathrm{CT}$ method was used to calculate changes in gene expression among the strains [105]. All experiments included both biological and technical replicates in triplicate. 


\section{Chemical libraries}

The St. Jude Children's Research Hospital compound collection, of approximately 800,000 compounds, was passed through a molecular weight filter which retained molecules weighing 200 to 300 Daltons. This set was further passed through structural alert filters obtained from the ChEMBL [179] database that removed compounds containing reactive and interfering substructures (e.g., PAINS compounds). The resulting set of molecules was clustered using the FCFP_4 fingerprints in Pipeline Pilot to obtain 10,000 most diverse molecular clusters. The molecules that were the cluster centers then formed the final "lead-like" set. To cover a different area of the chemical space than the lead-like set, molecules with molecular weight from 300 to 500 Daltons and aLogP values from 3 to 5 were obtained from the St. Jude collection. This set of molecules was further filtered to retain molecules having hydrogen bond donor counts less than or equal to 5, hydrogen bond acceptor counts less than or equal to 10 and sum of hydrogen bond donor and acceptor counts at least 3 . This set was next passed through the ChEMBL structural alert filters to remove interfering compounds and compounds with reactive substructures. Finally, the remaining molecules were clustered using the FCFP_4 fingerprints in Pipeline Pilot to obtain ten thousand most diverse compounds that formed the "drug-like" set.

The Prestwick library of 1280 small molecules was purchased from Prestwick Chemical. The FDA Approved Oncolytic library (147 compounds), the Natural Products Set V (419 natural products), and the NCI Diversity Set V (1593 compounds) were provided by the NIH Developmental Therapeutics Program. Finally, the 10,000 compounds from the Myria collection were purchased from Sigma-Aldrich. All library compounds were supplied at $10 \mathrm{mM}$ in DMSO in 96-well plate format. These were diluted further in DMSO to final concentration of $1 \mathrm{mM}$, and $1 \mu 1$ volumes dispensed into round-bottomed 96-well plates, or $0.2 \mu$ volumes were dispensed into 384-well plates, that were used for the chemical screens.

\section{Chemical screening}

The FP-labeled $C$. albicans strains expressing low, intermediate, reference, and high levels of the target protein were grown overnight in YPD at $30^{\circ} \mathrm{C}$, diluted to approximately $1 \times 10^{4}$ cells $/ \mathrm{ml}$ in YNB medium, and mixed 1:1:1:1 to create the fourmember expression pools. Nineteen microliters of the mixed cell suspension $\left(\sim 10^{3}\right.$ cells $)$ were then added to each well of the assay plates, resulting in final compound concentrations of $10 \mu \mathrm{M}$. After 24 and 48 hours of incubation at $30^{\circ} \mathrm{C}, \mathrm{OD}_{600 \mathrm{~nm}}$ and fluorescence intensity at the appropriate wavelengths were measured as described above. The relative fitness differential $(R d)$ was then calculated for each well as the $\log _{10} \mathrm{~T}_{\mathrm{Hi}} / \mathrm{T}_{\mathrm{LO}}$ fluorescence and converted to $\mathrm{Z}$ scores using the average and standard deviation of the $R d$ score of the minus drug controls across the whole collection. Hits were called based on a $Z$ score of $\geq(+3)$ and $\leq(-3)$. Wells with little detectable $C$. albicans growth $(\mathrm{OD} 600 \mathrm{~nm}$ of $\leq 0.1)$ were discarded from the analysis. 


\section{Restoring growth in the presence of hit compounds by supplementation of fatty acids}

The restoration of growth in the presence of the hit compounds was achieved by adding $2.5 \mathrm{mM}$ palmitic and oleic acids (16:1 and 18:1) dissolved in tergitol at 100x of the final concentration to YNB agar, with $1 \%$ tergitol as the mock treatment. The confirmed Ole $1 \mathrm{p}$ hits were then added to the agar at a final concentration of $25 \mu \mathrm{M}$. A cotton swab was used to streak the untagged Ole1p heterozygote across the plate. The plates were then incubated at $30^{\circ} \mathrm{C}$ for 48 hours and photographed at the 24 and 48 -hour timepoint. The restoration of growth was determined visually.

\section{Results}

\section{TAFiS provides high-throughput compatible screens to detect FAS and Ole1p inhibitors}

To generate $C$. albicans strains with differential susceptibility to FA synthesis inhibitors, we genetically altered the expression of either the FAS1 or OLE1 genes. In Saccharomyces cerevisiae, FAS1 influences the expression of FAS2 at the mRNA level through elements located within the FAS2 ORF [180] to keep a consistent stoichiometry between the subunits. Therefore changes in the mRNA for FAS1 are expected to alter the abundance of both FAS subunits in $C$. albicans. A strain that overexpresses the FAS complex (FASoE) was made by introducing an additional copy of FAS1 under the powerful and constitutively active $T E F 1$ promoter $\left(P_{T E F 1}\right)$ into a wild-type $C$. albicans strain $\left(F A S 1 / F A S 1+P_{T E F 1-F A S 1)}\right.$ (Figure 4-1A). To lower the expression of the wildtype (FAS ${ }_{\text {wT }}$ ), the first allele of $F A S 1$ was deleted and replaced with the $A R G 4$ selection marker to generate a FAS1/fas 1A:ARG4 heterozygote (FASHET). FAS1 is essential in $C$. albicans, and thus the second allele cannot be deleted without FA supplementation [88]. Therefore, to reduce transcription further, we attempted to replace the native promoter of the second FAS1 allele with several promoters, known to support varying levels of transcription [104]. Only one promoter, $P_{T E F 1}$, which typically drives a high level of expression under basal conditions, successfully replaced the endogenous promoter to

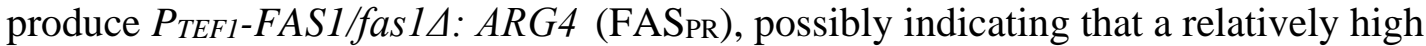
level of FAS1 transcription is necessary to support $C$. albicans viability. Essentially the same strategy was used to generate $C$. albicans strains with altered $O L E 1$ transcription, including an $O L E 1 / O L E 1+P_{T E F 1}-O L E 1$, an OLE1/ole1 $\triangle$ :URA3 heterozygous strain, and a $P_{A C T 1-O L E 1 / o l e 1 \triangle: U R A 3}$ strain, in which transcription of the second allele was controlled by the moderately strong promoter from the $A C T 1$ gene (Figure 4-1B).

Next, we compared the levels of each transcript in the engineered strains with a wild-type control strain (FAS1/FAS1 OLE1/OLE1) by RTqPCR. This revealed that when grown in YPD medium, both the FASOE and FASPR strains expressed approximately 4fold higher levels of the FAS1 transcript than the wild-type control, with the FASHET strain producing slightly less than the wild-type (Figure 4-2A). Thus, in total, a 

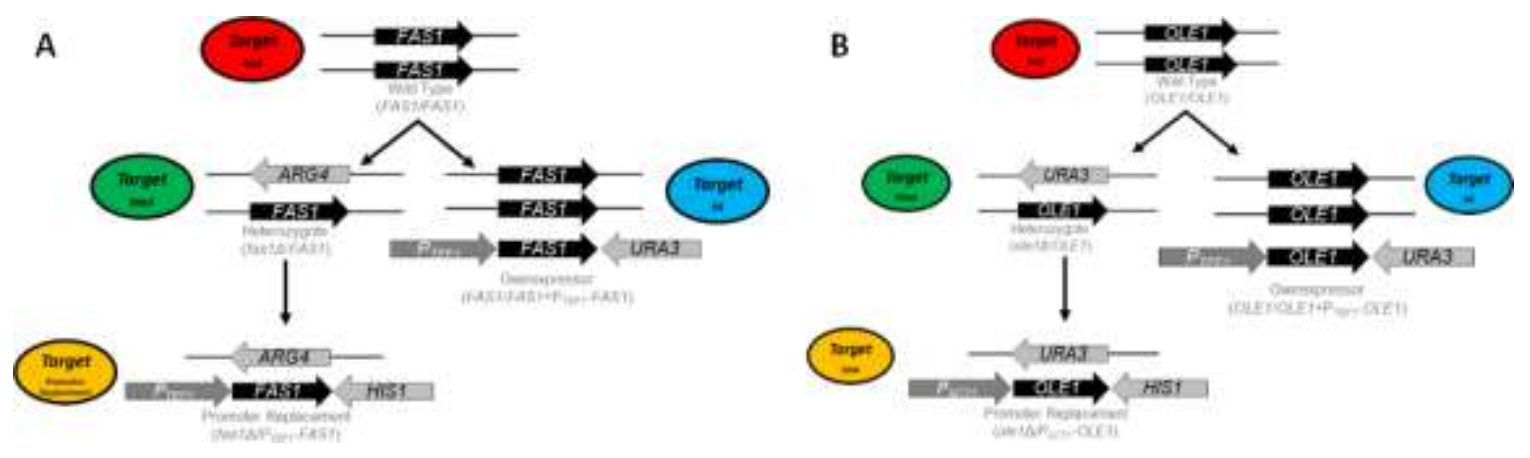

Figure 4-1. Construction of strains with altered FAS1 and $O L E 1$ expression. Schematic of the construction of strains with altered expression of (A) FAS1 or (B) OLE1. 

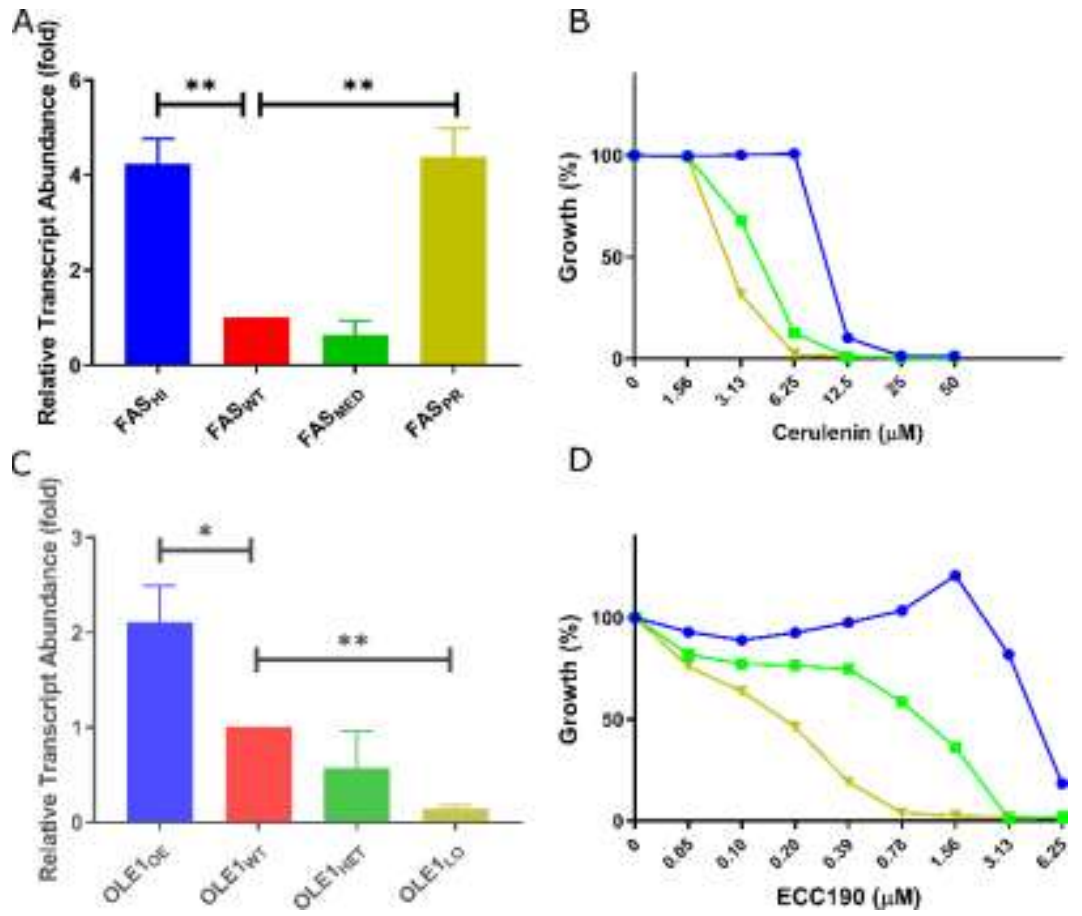

Figure 4-2. Modulating FAS1 and $O L E 1$ expression levels in Candida albicans affects susceptibility to cerulenin and ECC190 respectively.

(A) C. albicans strains were grown to exponential phase in YPD medium. Total RNA was extracted and then the (A) FAS1 or (C) OLE1 transcript abundance was determined by RTqPCR. Target gene transcript abundance was normalized to that of the ACT1 transcript abundance and then expressed relative to the target gene transcript abundance in the wild-type control (SC5314). These data are the average of three biological and technical replicates. B) C. albicans strains FAS1/FAS1+P TEF1-FAS1 (FASHI), FAS1/FAS1

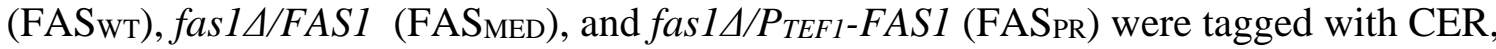
dTOM, GFP $\gamma$, and $\varphi$ YFP respectively, and were combined in equal proportions to create the FAS expression pool. Approximately $1 \times 10^{3}$ cells of the pool was inoculated into YNB medium in the wells of a 96-well plate in the presence of a serial dilution series of cerulenin. After 48 hours at $30^{\circ} \mathrm{C}$, fluorescence was quantified for all four FPs, and expressed as a percentage of fluorescence of the untreated wells. Values presented are the averages and standard deviations of technical triplicates and are representative of two

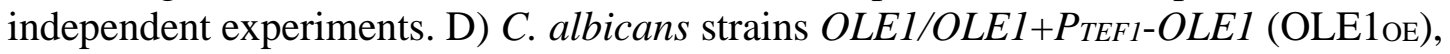
OLE1/OLE1 (OLE1wT), ole1 1/OLE1 (OLE1 $1 \mathrm{HET}$ ), and ole1 $\triangle / P_{A C T 1}-O L E 1$ (OLE1LO) were tagged with CER, dTOM, GFP $\gamma$, and $\varphi$ YFP respectively, and were combined in equal proportions to create the OLE1 expression pool. Approximately $1 \mathrm{X} 10^{3}$ cells of the pool was inoculated into YNB medium in the wells of a 96-well plate in the presence of a serial dilution series of ECC190. After 48 hours at $30^{\circ} \mathrm{C}$, fluorescence was quantified for all four FP's wavelengths, and expressed as a percentage of fluorescence of the untreated wells. Values presented are the averages and standard deviations of technical triplicates and are representative of two independent experiments. * indicates $\mathrm{P}<0.05$ and ** indicates $\mathrm{P}<0.001$ compared to the parent strain in the same condition using an unpaired student's t test. 
differential of just 4-fold was attained in FAS1 transcript abundance between the highest and lowest expressing strains. The OLE1/OLE1 + $P_{T E F 1-O L E 1}$ and $P_{A C T 1}-O L E 1 /$ ole 14 strains produced approximately 2- and 0.5-fold the amount of $O L E 1$ transcript compared to the wild-type, respectively (Figure 4-2C). Thus we designated OLE1/OLE1 + $P_{T E F 1^{-}}$ OLE1 as OLE1 ${ }_{\mathrm{HI}}$ OLE1/OLE1 as OLE1wT, OLE1/ole1 $\triangle$ as OLE1 $1_{\mathrm{HET}}$, and $P_{A C T 1^{-}}$ OLE1/ole1A as OLE1 Lo. However, the OLE1/ole1A strain produced about 5-fold less $O L E 1$ transcript than the wild-type, creating a total transcript differential of approximately 10-fold between the OLE1OE and OLE1 LO strains. For both genes, strains of each genotype were tagged through the introduction of an expression vector supporting the production of high-levels of a spectroscopically-distinct cytoplasmic fluorescent protein (FP) [104]. Finally, for each gene, we combined the labeled strains into two pools containing strains with either differential FAS1 or differential OLE1 transcription.

In order to demonstrate that the TAFiS methodology can be applied to identify inhibitors of FA synthesis, we sought to confirm that on-target inhibitors induced the expected dose-dependent population shift within the respective FAS1 or OLE1 pools, and this can be detected spectroscopically. The FAS expression pool was dispensed into 96well plates, at approximately 1 x $10^{4}$ cells per well, with increasing concentrations of cerulenin (CRL), a known FAS inhibitor [163], and incubated at $30^{\circ} \mathrm{C}$. After 48 hours, the fluorescent intensity of each tag was quantified to determine the relative abundance of each strain in the population when compared to the DMSO-alone control. CRL induced a population shift within the FAS1 pool as expected, but only over a narrow 2-fold range of concentrations between 3.13 and $6.25 \mu \mathrm{M}$ (Figure 4-2B). However, a population shift was observed in the $O L E 1$ pool over $\geq 64$-fold range of concentrations of ECC190

(Figure 4-2D), an Ole1p inhibitor [88]. Cerulenin did not induce the population shift that favored the highest expression strain in the Ole1p pool, nor did ECC190 induce a shift in the FAS expression pool (Figure 4-3). This confirmed that on-target inhibitors reliably and specifically induce the anticipated population shifts in the respective FAS and Ole1p expression pools. Fluorescence-based quantification of the population shifts induced within the FAS pool by $5 \mu \mathrm{M}$ cerulenin yielded $Z$-factor scores of $0.56 \pm 0.06$ in the 384-well plate format. Similar experiments with $5 \mu \mathrm{M}$ ECC190 and the Ole1p expression pool yielded Z'-factor of $0.70 \pm 0.03$. This indicates that both the FAS and Ole $1 \mathrm{p}$ based TAFiS assays can provide high quality and high-throughput compatible chemical screens to identify inhibitors of fungal FA biosynthesis.

\section{Identification of potential fatty acid biosynthesis inhibitors}

Initially, a total of 27,840 small molecule compounds were selected from the St. Jude Children's Research Hospital collection, enriched for lead-like and drug-like molecules. This collection was screened using both the Ole1p or FAS expression pools separately in unbuffered YNB medium, and compounds causing a statistically significant population shift versus the DMSO treated controls (as detected spectroscopically by measuring the FP tags) were identified. Relative fitness differentials (Rd) were calculated for each well as the $\log _{10}$ ratio of the FP intensity of the high expression strain to the FP 

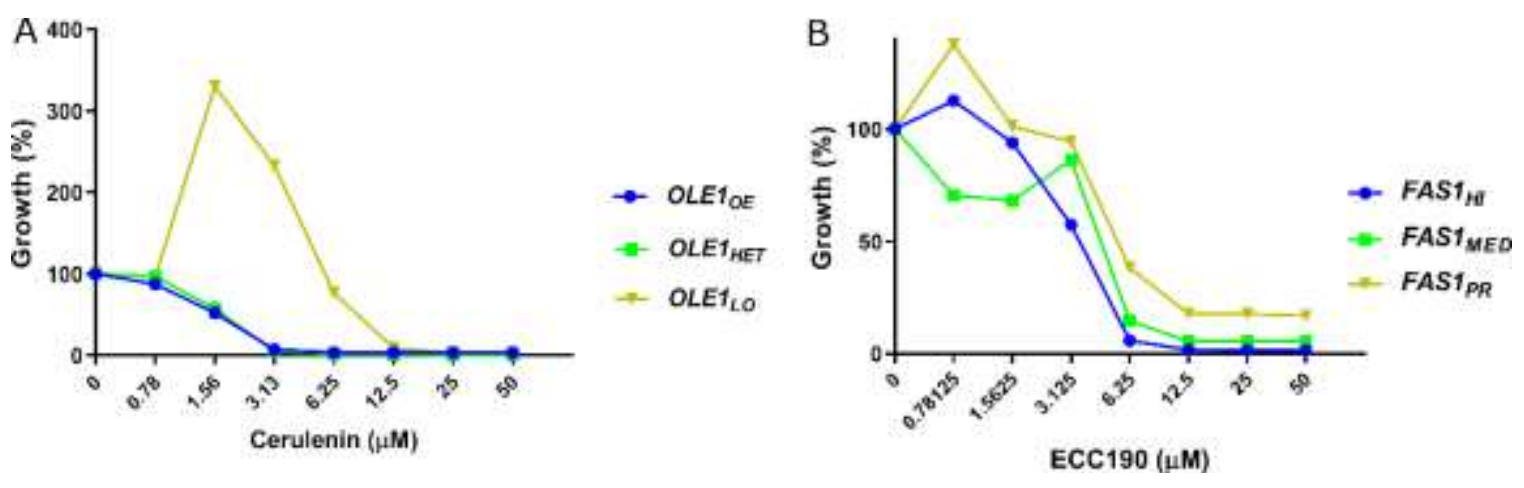

Figure 4-3. Off-target inhibitors do not cause population shifts that favor the high expression strain in the expression pools. (A) C. albicans strains OLE1/OLE1+P PEF1-OLE1 (OLE1OE), OLE1/OLE1 (OLE1wT), ole1 $\triangle / O L E 1$ (OLE1 $1 \mathrm{HET}$ ), and ole1 $\triangle / P_{A C T 1}-O L E 1$ (OLE1LO) were tagged with CER, $\mathrm{dTOM}, \mathrm{GFP} \gamma$, and $\varphi$ YFP respectively, and were combined in equal proportions to create the OLE1 expression pool. Approximately $1 \times 10^{3}$ cells of the combined pool was inoculated into YNB medium in the wells of a 96-well plate in the presence of a serial dilution of cerulenin. After 48 hours at $30^{\circ} \mathrm{C}$, fluorescence was quantified for all four FPs, and expressed as a percentage of fluorescence of the untreated wells. Values presented are the averages and standard deviations of technical triplicates and are representative of two independent experiments. (B) $C$. albicans strains FAS1/FAS1+P TEF1-FAS1 (FAS $_{\mathrm{HI}}$ ), FAS1/FAS1 (FASwT), fas1 $/$ /FAS1 (FAS $\mathrm{FED}_{\mathrm{W}}$ ), and

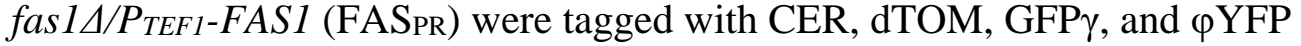
respectively, and were combined in equal proportions to create the FAS1 expression pool. Approximately $1 \times 10^{3}$ cells of the combined pool was inoculated into YNB medium in the wells of a 96-well plate in the presence of a serial dilution series of cerulenin. After 48 hours at $30^{\circ} \mathrm{C}$, fluorescence was quantified for all four FPs, and expressed as a percentage of fluorescence of the untreated wells. Values presented are the averages and standard deviations of technical triplicates and are representative of two independent experiments 
intensity of the low expression strain. The Rd value for each well was then converted to a Z-score based upon the mean and standard deviation of the DMSO control wells, and compounds with $Z$-scores of $\geq(+3)$ or $\leq(-3)$ were called as hits. Wells with a total optical density at $600 \mathrm{~nm}\left(\mathrm{OD}_{600 \mathrm{~nm}}\right)$ of $\leq 0.1$ were discarded from analysis as having populations insufficient to allow reliable detection of population shifts. Using these criteria, 22 compounds were identified as causing a population shift in the FAS pool, and a further 146 in the Ole1p pool. Of these, 59 of the Ole1p primary hits and all 22 FAS hits were selected for follow up based on the magnitude of the Z-score and availability (Table 4-2).

We screened an additional 13,421 compounds available in the Palmer Lab with both pools using the Natural Products Set V (419 natural compounds), the FDA Approved Oncolytic library (129 compounds), the Prestwick library (1280 compounds), the NCI Diversity Set V (1593 compounds), and a selection of 10,000 compounds from the Myria collection, a synthetically produced diversity library. Interestingly, neither expression pool identified any hits in the Oncolytic, Prestwick, or Diversity Set V libraries. However, the Ole1p pool identified 6 compounds from the Myria collection and 11 natural products as primary hits, while the FAS pool identified 4 compounds from the Myria collection, and 5 natural products as primary hits. Only one of the compounds identified by the Ole1p expression pool contained a hydrazide core. Interestingly, 6 hits, 3 each, for Ole1p and FAS had a similar core structure of 1,5-dihydro-2H-dipyridol[1,2a:2',3"-d]pyrimidine (Table 4-3). Unfortunately, we were unable to acquire any of these compounds for follow up assays.

Follow up dose-response assays were conducted with each of the 59 Ole1p and 22 FAS selected hit compounds using the respective Ole1p and FAS expression pools, and the relative fluorescence intensity of each individual strain plotted against concentration. To eliminate compounds that interfere with the detection of the fluorescent tags, we also utilized an inversely labeled pool where the FP tags are switched i.e. FASoE tagged with $\varphi$ YFP and FASPR tagged with CER. None of the 22 hit compounds for the FAS expression pool caused reproducible population shifts in the follow-up pooled doseresponse, with 3 of the 22 having fluorescent interference. Of the 59 Ole $1 p$ hits tested, 33 failed to induce the anticipated dose-dependent population shifts, and an additional 10 interfered with fluorescence detection, and were discarded as false positives. The remaining 16 caused significant and dose-dependent population shifts in the Ole1p pool (Figure 4-4). Interestingly, 11 of the 16 confirmed hits had a hydrazide moiety at the center of the molecule, potentially indicating a novel scaffold for future development.

To determine if these compounds have growth inhibitory activity on wild-type $C$. albicans, we determined their relative antifungal potency upon SC5314, a wild-type clinical isolate [149], using the standard CLSI broth microdilution protocol [181]. Only 2 of the 16 compounds, FAI354 and FAI409, were able to inhibit the growth of SC5314 at concentrations below $50 \mu \mathrm{M}$ in RPMI pH 7 medium (MIC50 3.125 and $6.25 \mu \mathrm{M}$ respectively). To determine if the Ole1p hits had $\mathrm{pH}$-dependent activity, we tested each compound's ability to inhibit growth in YNB pH 5, 6, and 7. All 16 Ole1p hits had 
Table 4-2. Description of screening results.

\begin{tabular}{llcccc}
\hline $\begin{array}{c}\text { Target } \\
\text { Protein }\end{array}$ & \multicolumn{1}{c}{ Library } & $\begin{array}{c}\text { Number of } \\
\text { Compounds } \\
\text { Screened }\end{array}$ & $\begin{array}{c}\text { Primary } \\
\text { Hits } \\
\text { Identified }\end{array}$ & $\begin{array}{c}\text { Primary } \\
\text { Hits } \\
\text { Obtained }\end{array}$ & $\begin{array}{c}\text { Primary } \\
\text { Hits } \\
\text { Confirmed }\end{array}$ \\
\hline Ole1p & Saint Jude Chemical & 27840 & 146 & 59 & 16 \\
& Collection & & & & \\
& Myria & 10000 & 6 & 0 & 0 \\
& Prestwick & 1280 & 0 & 0 & 0 \\
& FDA Oncolytic & 129 & 0 & 0 & 0 \\
& NCI Diversity Set V & 1593 & 0 & 0 & 0 \\
& 384 Natural Product & 419 & 11 & 0 & 0 \\
& Saint Jude Chemical & 27840 & 22 & 22 & 0 \\
& Collection & & & & \\
& Myria & 10000 & 4 & 0 & 0 \\
& Prestwick & 1280 & 0 & 0 & 0 \\
& FDA Oncolytic & 129 & 0 & 0 & 0 \\
& NCI Diversity Set V & 1593 & 0 & 0 & 0 \\
& 384 Natural Product & 419 & 5 & 0 & 0 \\
\hline
\end{tabular}


Table 4-3. Structures of compounds from the Myria collection identified as causing statistically significant population shifts in either FAS or Ole1p expression pools.

Oxpression



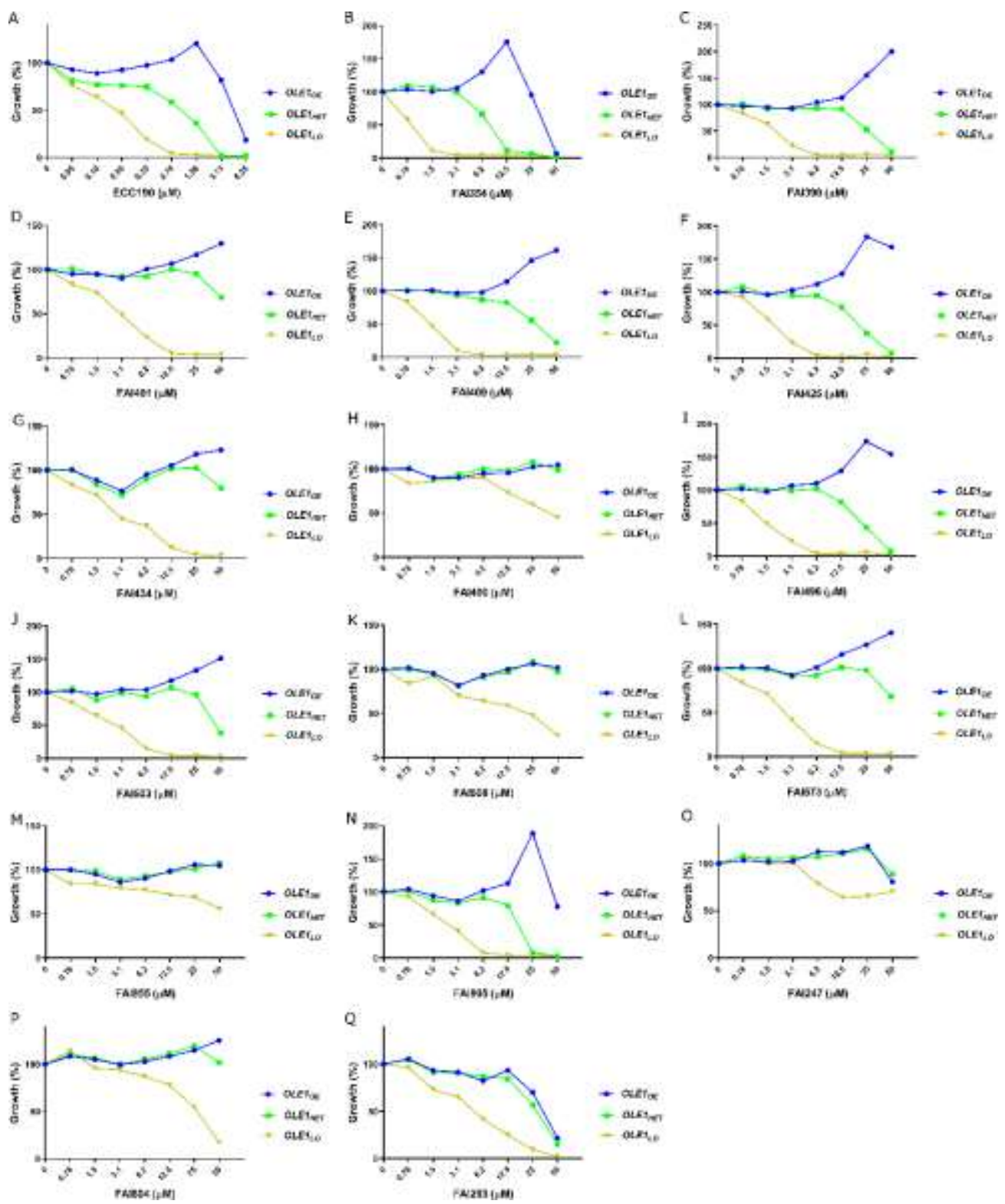

Figure 4-4. The primary hits from the Ole1p screens induce the expected population shift in the $O L E 1$ expression pool.

C. albicans strains OLE1/OLE1+PTEFI-OLE1 (OLE1oe), OLE1/OLE1 (OLE1wT),

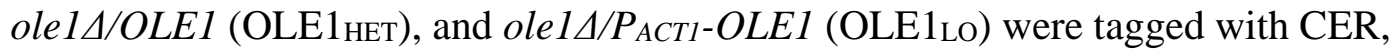
dTOM, GFP $\gamma$, and $\varphi$ YFP respectively, and were combined in equal proportions to create the OLE1 expression pool. Approximately $1 \times 10^{3}$ cells of the combined pool was inoculated into YNB medium in the wells of a 96-well plate in the presence of a serial dilution of (A) ECC190, (B) FAI354, (C) FAI390, (D) FAI401, (E) FAI409, (F) FAI425, (G) FAI434, (H) FAI490, (I) FAI496, (J) FAI503, (K) FAI508, (L) FAI573, (M) FAI855, (N) FAI805, (O) FAI247, (P) FAI804, and (Q) FAI293. After 48 hours at $30^{\circ} \mathrm{C}$, fluorescence was quantified for all four FPs, and expressed as a percentage of fluorescence of the untreated wells. Values presented are the averages and standard deviations of technical triplicates and are representative of two independent experiments. 
increased growth inhibition as the $\mathrm{pH}$ of the medium increased (Figure 4-5). In addition, when we tested the confirmed hits in TAFiS dose-response assays with the Ole1p expression pool, they induced the expected population shift at a lower concentration with increasing $\mathrm{pH}$. Another observation was that each compound has a lower $\mathrm{MIC}_{50}$ in YNB medium buffered to $\mathrm{pH} 7$ than in RPMI pH 7 medium. This indicates that not only do these hits have $\mathrm{pH}$-dependent activity, but also medium-dependent activity.

\section{The supplementation with unsaturated fatty acids restores the growth of Candida albicans's growth in the presence of four hydrazide containing screening hits}

To further confirm that these compounds affect the production of UFAs, we determined if supplementation of the medium with palmitic and oleic acids (16:1 and 18:1) was sufficient to restore $C$. albicans growth in the presence of inhibitory concentrations of each compound. Unfortunately, 12 of the compounds did not inhibit the growth of even the $O L E 1$ heterozygote strain under the conditions tested (on YNB medium agar). However, of the remaining 4 compounds that inhibited $C$. albicans growth, the UFA supplements suppressed the growth inhibitory effects of all 4 hit compounds (FAI354, FAI409, FAI425, FAI496) (Figure 4-6), interestingly all four contained the hydrazide moiety. This further supports that these compounds inhibit growth by interfering with the production of UFA.

\section{The Ole1p inhibitors are structurally related and rely on a hydrazide moiety for activity}

In an effort to explore the structure-activity relationship (SAR) of the two confirmed hits that had antifungal activity against wild-type C. albicans (FAI354 and FAI409) both of which contained the hydrazide connected to an aromatic ring (Table 4-4). We kept the thiadiazole and isoxazole rings constant and began modifying the aromatic ring connected to the hydrazide moiety. Since both leads have fluorine atoms in the ortho $(o)$ position of the aromatic ring, we looked at para (p) (FAI4427 and FAI4428) and $o, p$-difuoro compounds (FAI4439 and FAI4440, Figure 4-7). The isoxazole-derived compounds did show a slight shift from an $\mathrm{MIC}_{50}$ of 6.25 to $3.125 \mu \mathrm{M}$ for the $p$ fluoro compound FAI4427, but the thiadiazole maintained the same potency regardless of fluorine substitution. Removing all functional groups only resulted in the $\mathrm{MIC}_{50}$ of the isoxazole derivative increasing to $12.5 \mu \mathrm{M}$, while the $\mathrm{MIC}_{50}$ of the thiadiazole remained unchanged (data not shown). Indeed, when looking at electron donating groups (methyl and methoxy), the only significant change in $\mathrm{MIC}_{50}$ was observed with the $o$ methoxy isoxazole FAI4491, whose potency decreased to $50 \mu \mathrm{M}$.

Having looked at the 1,2,3-thaidiazole and the 1,2-oxazole moiety of FAI354 and FAI409, we evaluated the central hydrazide core for its effect on antifungal activity. Interestingly, when the aryl $\mathrm{NH}$ was replaced with a methylene group, both series lost all potency and nearly all activity (MIC $50>50 \mu \mathrm{M}$, FAI4437 and FAI4438, Figure 4-7). Altering the benzyl amine to a heterocyclic amide did not restore any potency or activity 
A
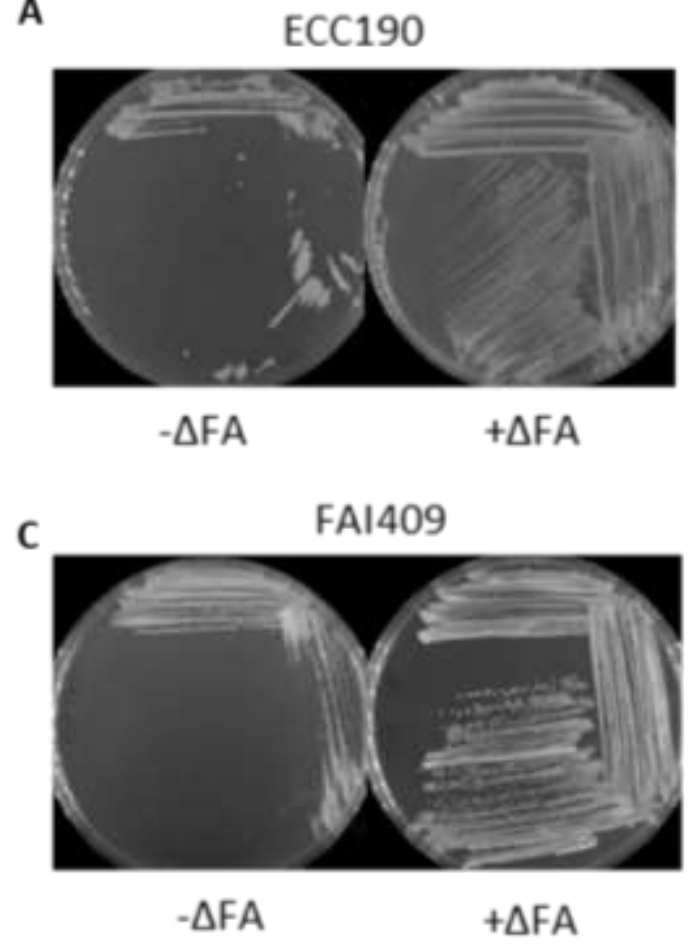

B

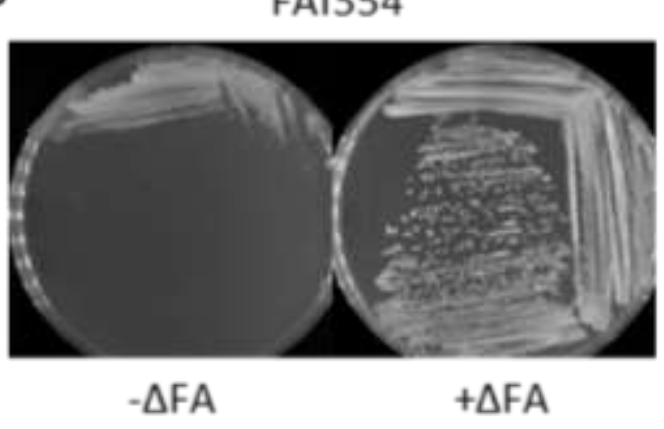

D

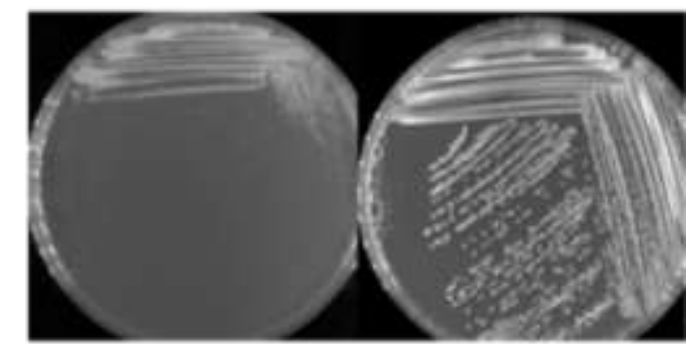

$-\triangle F A$

$+\triangle F A$

$\mathbf{E}$

FAl496

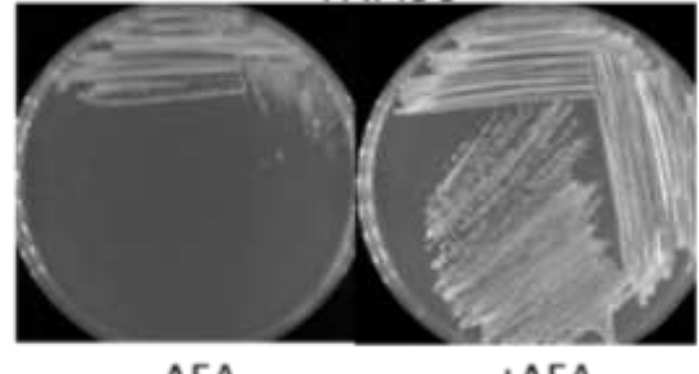

$-\triangle \mathrm{FA}$

$+\triangle \mathrm{FA}$

Figure 4-5. The presence of unsaturated fatty acids restores the growth of Candida albicans in the presence of Ole1p inhibitors.

The restoration of growth in the presence of the hit compounds was achieved by adding palmitic and oleic acids (16:1 and 18:1) dissolved in tergitol at 100x of the final concentration to YNB medium agar, with $1 \%$ tergitol as the mock treatment. The confirmed Ole1p hits were then added to the cooled molten agar at a final concentration of $25 \mu \mathrm{M}$. A cotton swab was used to streak the untagged $O L E 1$ heterozygote across the plate and then the plates were incubated at $30^{\circ} \mathrm{C}$ for 48 hours. The plates were then photographed at the 24 and 48-hour timepoint. The restoration of growth was determined visually by the reduction in the growth inhibition. 

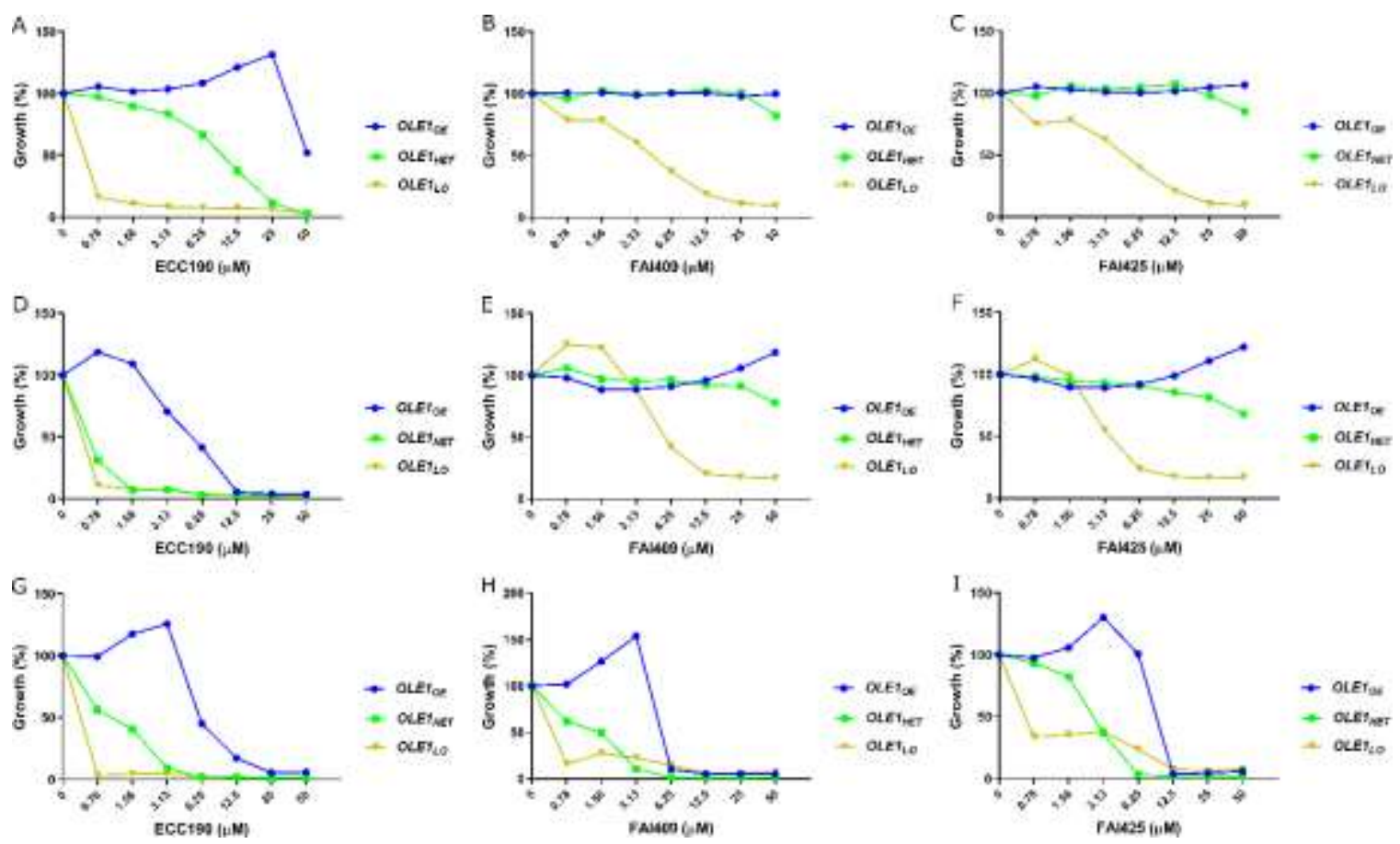

Figure 4-6. Ole1p inhibitors have pH-dependent activity on Candida albicans. C. albicans Ole1p strains OLE1/OLE1+P PEF1-OLE1 (OLE1 oe), OLE1/OLE1 (OLE1wT), ole1 1 /OLE1 (OLE1 $\mathrm{HET}$ ), and ole1 $/ \mathrm{P}_{A C T 1}$-OLE1 (OLE1LO) were tagged with CER, dTOM, GFP $\gamma$, and $\varphi$ YFP respectively, and were combined in equal proportions to create the Ole1p expression pool. Approximately $1 \times 10^{3}$ cells of the combined pool was inoculated into YNB medium buffered to $\mathrm{pH} 5$ (A, B, and C), 6 (D, E, and F), or 7 (G, H, and I) in the wells of a 96-well plate in the presence of a serial dilution of ECC190 (A, D, and G), FAI409 (B, E, and H), and FAI425 (C, F, and I). After 48 hours at $30^{\circ} \mathrm{C}$, fluorescence was quantified for all four FP's wavelengths, and expressed as a percentage of fluorescence of the untreated wells. Values presented are the averages and standard deviations of technical triplicates and are representative of two independent experiments. 
Table 4-4. Representative compounds from the structure-activity relationship testing of FAI354 and FAI409.

\begin{tabular}{|c|c|c|c|}
\hline Compound & Structure & $\begin{array}{l}\text { Fold-Differential of } \\
\text { OLE1 } 1_{\mathrm{OE}} \text { to OLE1 } 1_{\mathrm{LO}}\end{array}$ & $\mathrm{MIC}_{50}(\mu \mathrm{M})$ \\
\hline FAI4424 & & $>256$ & 6.25 \\
\hline FAI4425 & & $>256$ & 3.125 \\
\hline FAI4427 & & $>256$ & 3.125 \\
\hline FAI4428 & & $>256$ & 3.125 \\
\hline FAI4437 & & $>8$ & $>50$ \\
\hline FAI4438 & & 0 & $>50$ \\
\hline FAI4439 & & $>256$ & 6.25 \\
\hline FAI4440 & & $>256$ & 3.125 \\
\hline FAI4487 & & 0 & $>50$ \\
\hline FAI4488 & & 0 & $>50$ \\
\hline FAI4489 & & $>2$ & $>50$ \\
\hline FAI4490 & & $>4$ & $>50$ \\
\hline FAI4491 & & $>32$ & 50 \\
\hline
\end{tabular}



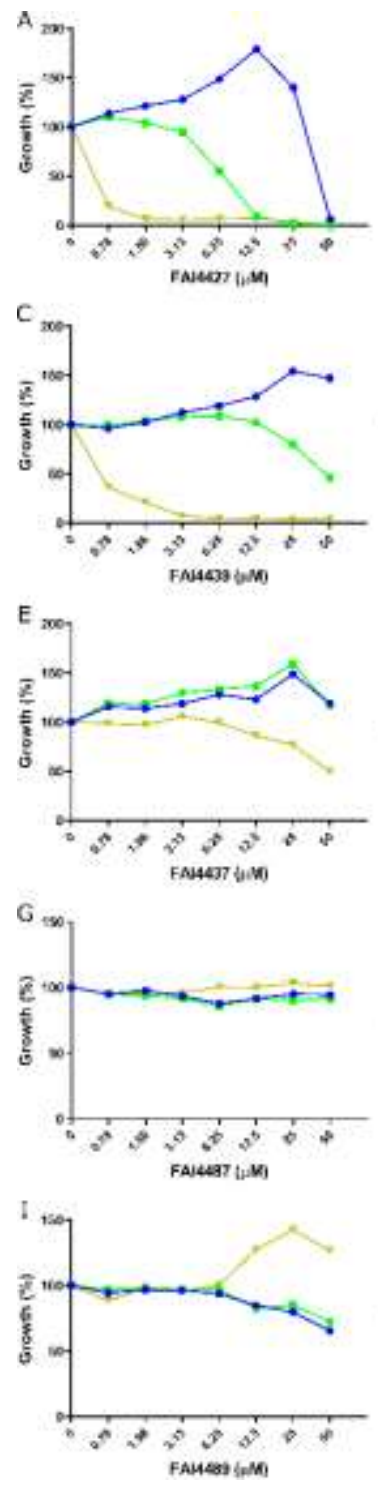
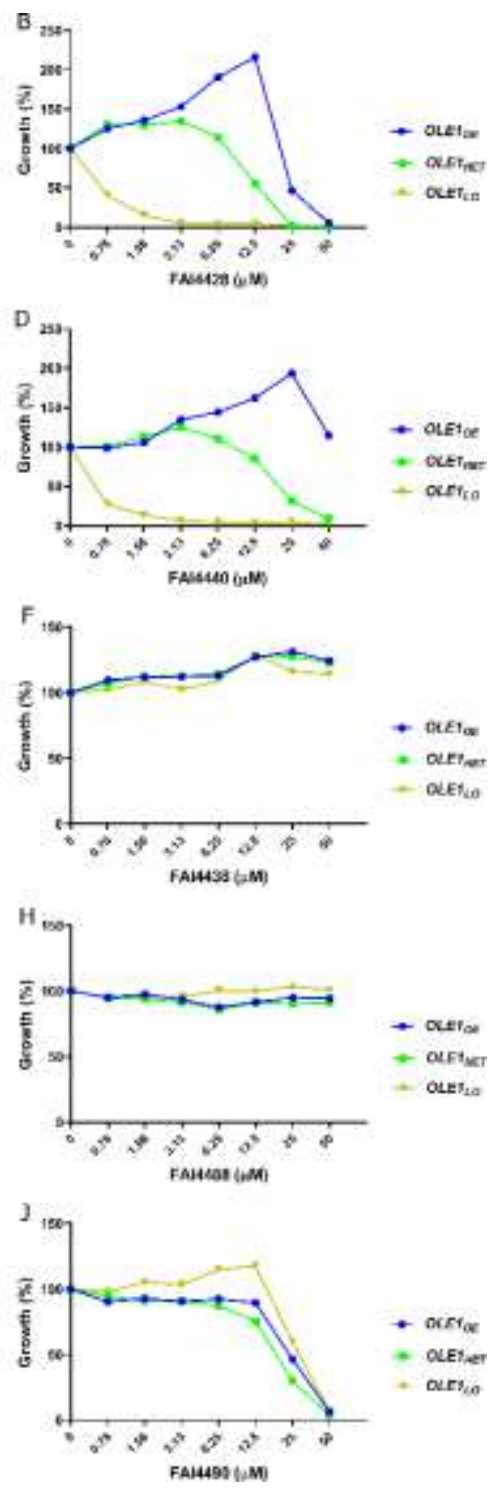

Figure 4-7. Substitutions on the aromatic ring do not alter the on-target activity of Ole1p hits, but the hydrazine core is essential.

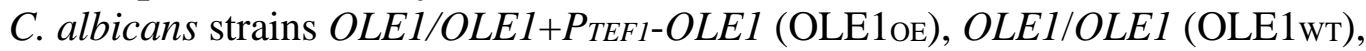
ole1 $1 / O L E 1$ (OLE1 $\mathrm{HET}$ ), and ole1 $/$ P $_{A C T 1}$-OLE1 (OLE1 Lo) were tagged with CER, dTOM, GFP $\gamma$, and $\varphi$ YFP respectively, and were combined in equal proportions to create the OLE1 expression pool. Approximately $1 \times 10^{3}$ cells of the combined pool was inoculated into YNB medium in the wells of a 96-well plate in the presence of a serial dilution of (A) FAI4427, (B) FAI4428, (C) FAI4439, (D) FAI4440, (E) FAI4437, (F) FAI4438, (G) FAI4487, (H) FAI4488, (I) FAI4489, and (J) FAI4490. After 48 hours at $30^{\circ} \mathrm{C}$, fluorescence was quantified for all four FPs, and expressed as a percentage of fluorescence of the untreated wells. Values presented are the averages and standard deviations of technical triplicates and are representative of two independent experiments. 
to the level of the original molecule (FAI4487-FAI4490, Figure 4-7). Even modification of the hydrazide group directly deactivated the compounds (Figure 4-7). Replacing the aryl N-H with an N-methyl resulted in analogs that failed to induce population shifts in the $O L E 1$ expression pool. Therefore we conclude that the hydrazide core is essential to the potency and the chemical-target interaction.

\section{The Ole1p inhibitor ECC190 contains a 1,2,4-triazolidine-3-thione core that degrades to produce acylhydrazones}

As previously stated, Becker and colleagues identified a family of Ole1p inhibitors that shared a 1,2,4-triazolidine-3-thione core. We hypothesized that the heterocycle group in the triazolidine compounds, described in the Becker study, might degrade to a hydrazone (Figure 4-8A) and therefore closely resemble a collection of potent antifungals (of unknown mechanism) described by Carradori et al. [90], and are structurally similar to our own hydrazine compounds that interact with the Ole $1 \mathrm{p}$ desaturase. We sought to determine the structure-activity relationship of ECC190 and to confirm that it acts through the same mechanism as the acylhydrazone antifungals described by Carradori et al.

To determine if the 1,2,4-triazolidine-3-thione antifungals broke down to form acylhydrazones, we generated an analog of ECC190, FAI000 to facilitate NMR analysis (Table 4-5). FAI000 was dissolved in DMSO and allowed to degrade over 48 hours at room temperature. After 16, 24, and 48 hours the NMR spectrum was determined (Figure 4-8B). The resulting spectrum revealed the breakdown of the 1,2,4 triazolidine core into a hydrazone, confirming our hypothesis. To provide further evidence that the hydrazone was the active form of ECC190, we resynthesized the acylhydrazone product, termed FAI101, and compared its antifungal potency to ECC190 against a wild-type $C$. albicans strain. Under the conditions of the CLSI antifungal susceptibility testing, both FAI101 and ECC190 inhibited the growth of a wild-type C. albicans at similar concentrations ( $\mathrm{MIC}_{50} 3.125 \mu \mathrm{M}$ ) (Figure 4-9). This confirmed that the hydrazone derivative retained antifungal activity, and suggested that it may be the active form.

\section{The acylhydrazone derivatives of the 1,2,4-triazolidine scaffold act through the same molecular mechanism}

In order to confirm that these compounds that act upon the Ole1p desaturase, we utilized the Ole1p TAFiS assay similarly to the SAR study with FAI354 and FAI409 (Table 4-4). FAI101 induced the same population shift that was observed with ECC190, indicating that FAI101's antifungal activity is dependent upon the expression OLE1, consistent with the same mechanism of action. To ensure that FAI101 did not interfere with fluorescent detection, we incubated it with the reciprocally tagged OLE1 expression pool. FAI101 induced the expected population shift in both the original and reciprocal pools, i.e. favoring the highest expression strain regardless of the FP, indicating that FAI101 does not interfere with fluorescent detection. To further confirm that the 
A

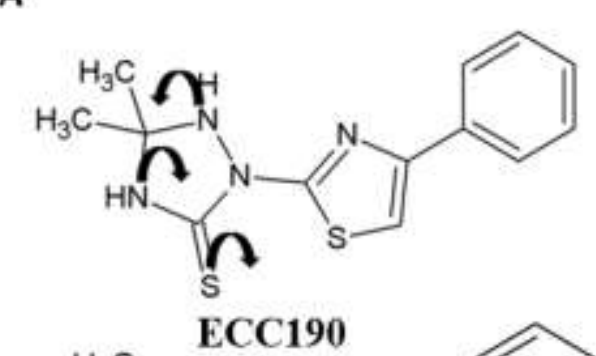<smiles>CC(C)=NN(C(=N)S)c1nc(-c2ccccc2)cs1</smiles>

Intermediate<smiles>CC(C)=NNc1nc(-c2ccccc2)cs1</smiles>

Hydrazone Product
B
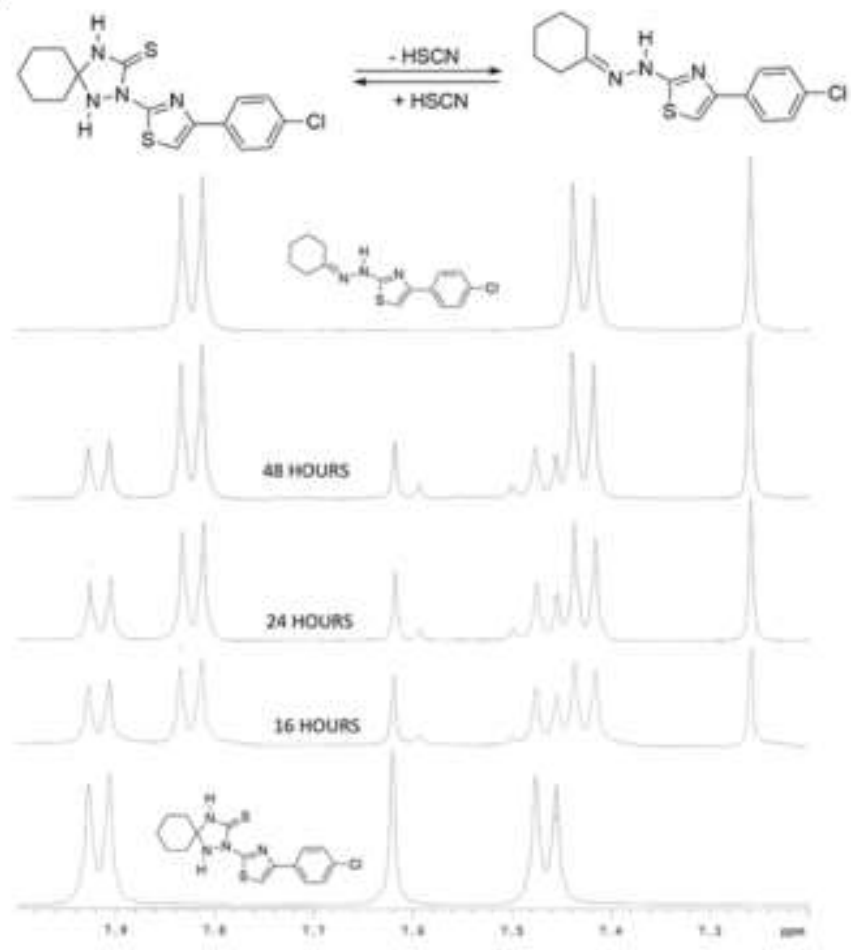

Figure 4-8. ECC190 degrades into a hydrazone containing compound.

Proposed mechanism for the degradation of ECC190 into the FAI101. (B) NMR spectrum of the ECC190 analog degrading to the expected hydrazone containing compound. 
Table 4-5. Activity and potency of the analogs of ECC190 and FAI101.

\begin{tabular}{|c|c|c|c|}
\hline Identifier & Structure & $\begin{array}{l}\text { Fold-Differential of } \\
\text { OLE1 } 1_{\text {oE }} \text { to OLE1 } 1 \text { LO }\end{array}$ & $\mathrm{MIC}_{50}(\mu \mathrm{M})$ \\
\hline ECC190 & & $>64$ & 3.125 \\
\hline FAI101 & & $>64$ & 3.125 \\
\hline FAI001 & & $>2$ & 25 \\
\hline FAI002 & & $>32$ & $>50$ \\
\hline FAI035 & & 0 & $>50$ \\
\hline FAI036 & & 0 & $>50$ \\
\hline FAI045 & & 0 & $>50$ \\
\hline FAI102 & & $>126$ & 1.56 \\
\hline FAI103 & & $>126$ & 3.125 \\
\hline FAI104 & & $>8$ & 3.125 \\
\hline FAI105 & & $>8$ & 3.125 \\
\hline FAI106 & & $>16$ & 6.25 \\
\hline FAI107 & & $>64$ & 1.56 \\
\hline FAI108 & & $>16$ & 25 \\
\hline
\end{tabular}


Table 4-5. (Continued).

\begin{tabular}{|c|c|c|c|}
\hline Identifier & Structure & $\begin{array}{l}\text { Fold-Differential of } \\
\text { OLE1 OE to OLE1 LO }\end{array}$ & $\mathrm{MIC}_{50}(\mu \mathrm{M})$ \\
\hline FAI109 & & $>32$ & 3.125 \\
\hline FAI110 & & $>64$ & 25 \\
\hline FAI111 & & $>16$ & 25 \\
\hline FAI121 & & $>64$ & 3.125 \\
\hline FAI131 & & $>8$ & 50 \\
\hline FAI141 & & $>16$ & $>50$ \\
\hline FAI151 & & $>32$ & $>50$ \\
\hline FAI156 & & $>16$ & $>50$ \\
\hline
\end{tabular}

The fold-differential of OLE1OE to OLELo is a measure of the relative activity of each compound. 


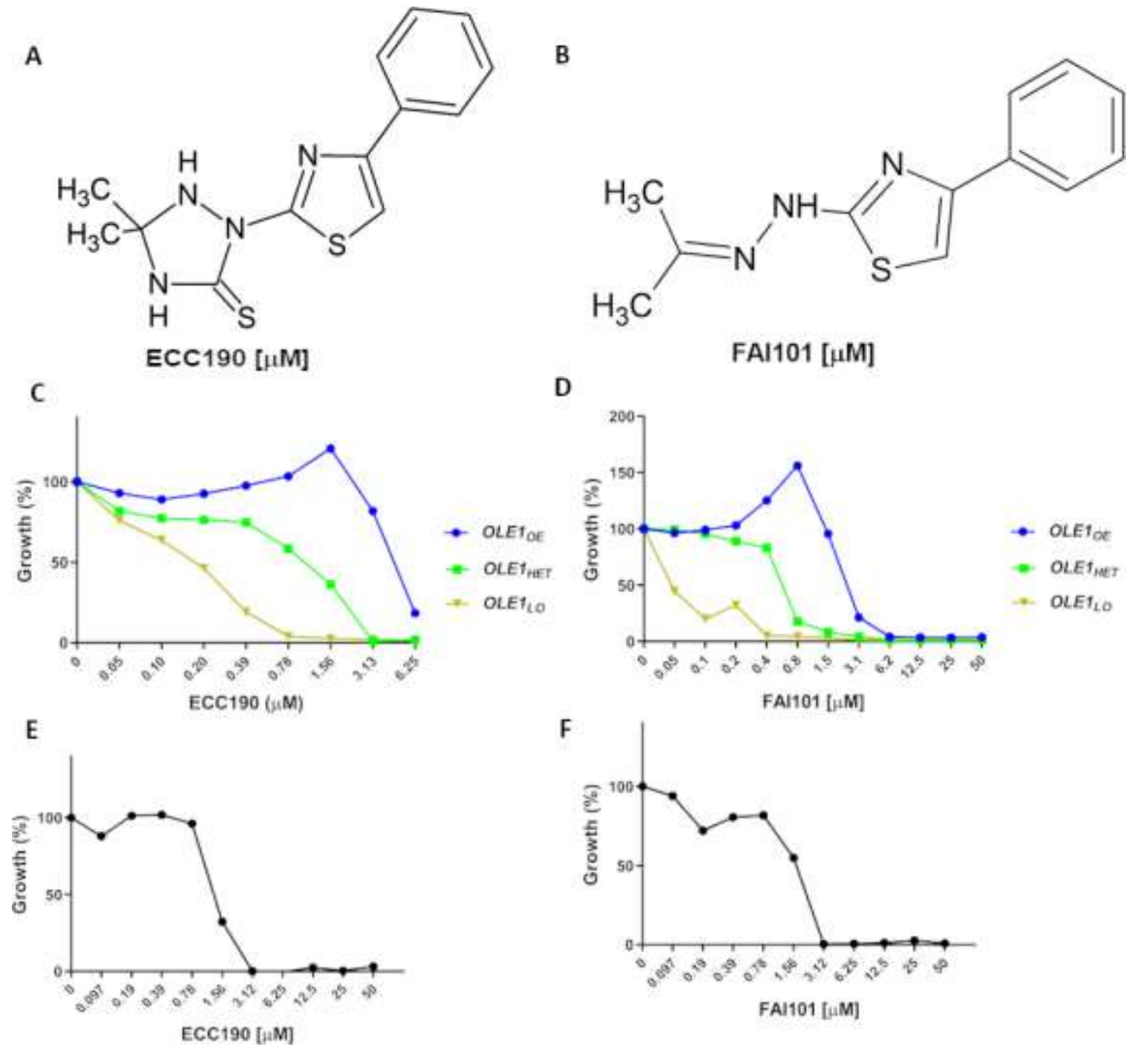

Figure 4-9. Structure and analysis of ECC190 and FAI101.

Structures of (A) ECC190 and (B) FAI101. C. albicans Ole1p strains

OLE1/OLE1+P PEF1-OLE1 (OLE1OE), OLE1/OLE1 (OLE1wT), ole1 $/$ OLE1 (OLE1HET),

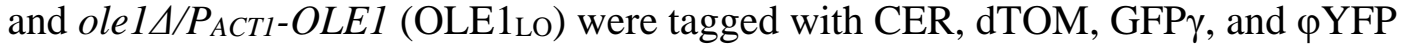
respectively, and were combined in equal proportions to create the Ole1p expression pool. Approximately $1 \times 10^{3}$ cells of the pool was inoculated into YNB medium in the wells of a 96-well plate in the presence of a serial dilution series of (C) ECC190 or (D) FAI101. After 48 hours at $30^{\circ} \mathrm{C}$, fluorescence was quantified for all four FP's wavelengths, and expressed as a percentage of fluorescence of the untreated wells. Approximately 1 X $10^{3}$ cells of OLE1wT was inoculated into RPMI $1640 \mathrm{pH} 7$ and then seeded into a 96-well plate in the presence of a serial dilution series of (E) ECC190 or (F) FAI101 and incubated at $35^{\circ} \mathrm{C}$. After 48 hours the absorbance at OD600nm was quantified and expressed as a percentage of the DMSO control. Values presented are the averages and standard deviations of technical triplicates and are representative of two independent experiments. 
acylhydrazone derivatives block UFA biosynthesis, we tested if supplementing the growth medium with $2 \mathrm{mM}$ palmitic and $2 \mathrm{mM}$ of oleic acid was sufficient to restore $C$. albicans growth in the presence of growth inhibitory concentrations of FAI101. Indeed, the UFA supplements rescued fungal growth in the presence of either ECC190 or FAI101 (Figure 4-10), further supporting that FAI101 has the same mechanism of action as ECC190. Therefore, we explored the SAR of ECC190, and the acylhydrazone, FAI101.

\section{The presence and identity of the thiazole linker is important for potency of the $1,2,4-$ triazolidine scaffold}

To determine if the thiazole linker group was essential for inhibition, we synthesized analogs that removed this linker, joining the 1,2,4 triazolidine and the phenyl ring directly. Removal of the thiazole group resulted in a molecule, FAI002, that had an 8 -fold increase in the $\mathrm{MIC}_{50}$. However, FAI002 retained the ability to induce population shifts, which indicate that it retained on-target activity. This loss of potency but not activity indicates that removal of the thiazole linker group does not alter the compound's molecular target, but reduces the scaffold's ability to inhibit fungal growth. Since the presence of the linker seems important to the potency of the scaffold, we determined if the identity of the linker affected the activity. We generated an analog, FAI001, which replaced the linker with a methyl group. Replacement of the thiazole linker resulted in a greater loss of potency (16-fold increase in the MIC50) than the complete removal, but this analog retained on-target activity as well. Therefore, it would seem that the presence and identity of the linker group are crucial to the potency of the molecule but have no effect on the compound's ability to engage the molecular target.

\section{The size and aromaticity of the phenyl ring are important for the acylhydrazone to interact with Ole1p and inhibit the growth of Candida albicans}

To determine the effect of hydrophilic groups on the phenyl ring, we synthesized analogs that placed a nitro group (FAI036) or a carboxyl group (FAI035) at the paraposition of the phenyl ring. Neither of these analogs had detectable growth inhibition at the concentrations tested (>16-fold increase in the MIC50), and these analogs failed to induce population shifts in the OLE1 expression pool, indicating that the hydrophilic additions eliminate the activity of the acylhydrazone.

To determine if the phenyl ring needs to be aromatic, we generated an analog that replaced the phenyl ring with cyclohexane (FAI045). This compound had an 8-fold increase in the $\mathrm{MIC}_{50}$ and failed to induce a population shift in the pooled dose responses, indicating the compound inhibits growth through a different mechanism. This result demonstrates that the aromatic structure of the phenyl group is necessary for the compound to function as an Ole1p inhibitor.

To determine if the size of the aromatic structure is important, we produced an analog that altered the phenyl group to a naphthalene group (FAI111). These analogs 


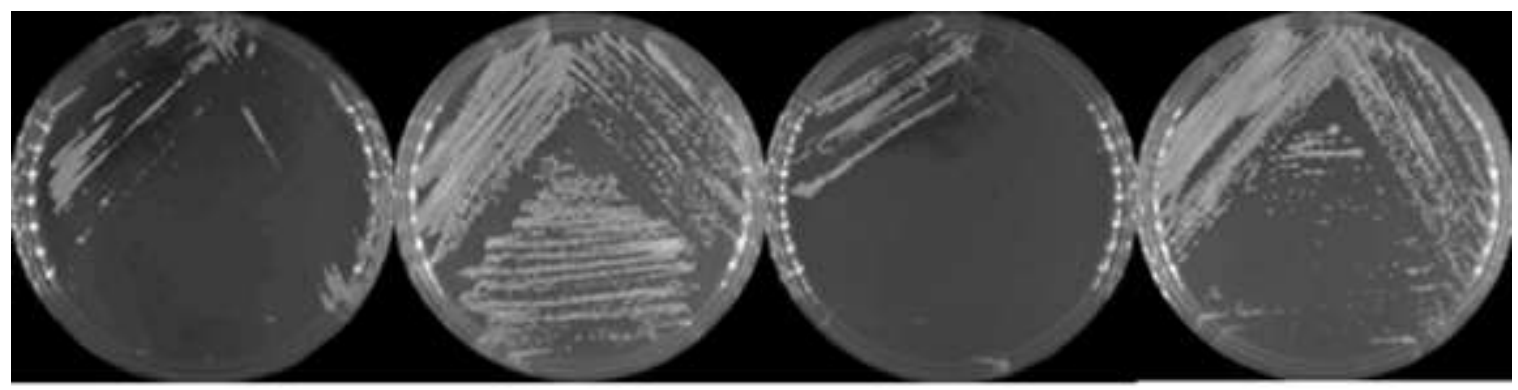

ECC190 $\quad$ ECC190 + FA $\quad$ FAl101 $\quad$ FAl101 + FA

Figure 4-10. Restoration of growth in the presence of inhibitors with the addition of unsaturated fatty acids.

The restoration of growth in the presence of the hit compounds was achieved by adding palmitic and oleic acids (16:1 and 18:1) dissolved in tergitol at 100x of the final concentration to YNB medium agar, with $1 \%$ tergitol as the mock treatment. The confirmed Ole1p hits were then added to the cooled molten agar at a final concentration of $25 \mu \mathrm{M}$. A cotton swab was used to streak the untagged Ole1p heterozygote across the plate and then the plates were incubated at $30^{\circ} \mathrm{C}$ for 48 hours. The plates were then photographed at the 24 and 48 -hour timepoint. The restoration of growth was determined visually by the reduction in the growth inhibition. 
retained their ability to induce population shifts in the $O L E 1$ expression pool but failed to inhibit the growth of a wild-type $C$. albicans strain ( >16-fold increase in MIC50), indicating that the aromatic structure is necessary, though the size is limited.

To determine the effect of halogen substitutions on the phenyl ring we generated analogs with meta- (FAI121), para- (FAI131), meta-/para- (FAI141), ortho-/para(FAI151), and ortho-/meta-/para-substitutions (FAI156). The compounds with metasubstitutions retained activity but had an 8-fold increase in the $\mathrm{MIC}_{50}$. The analogs with para-substitutions retained both potency and activity. In contrast, compounds with meta/para-substitutions retained the activity but had a 16-fold increase in the MIC50 against $C$. albicans. Similarly, analogs with either ortho-/para-substitutions, or substitutions at all three positions, retained their ability to cause population shifts in the OLE1 expression pool but lost their ability to inhibit the growth of a wild-type $C$. albicans strain (>16-fold increase in the $\mathrm{MIC}_{50}$ against $C$. albicans). Therefore we conclude that none of these substitutions affected the activity but that only para-substitutions are acceptable to retain the potency of the scaffold.

\section{The acylhydrazone's ability to interact with Ole1p and inhibit Candida albicans growth requires hydrophobic functional groups attached directly to the hydrazone core}

We investigated the two functional groups (R groups) that are directly attached to the hydrazone core and generated analogs with different lengths of hydrophobic chains as well as hydrophilic groups. We found that small hydrophobic chains on either or both of the R groups had no noticeable effect on the activity or potency of the molecule (FAI102, FAI103, FAI104, FAI105). However, when the $\mathrm{R}_{1}$ and $\mathrm{R}_{2}$ groups form a cyclopentane (FAI106), there is a 2-fold increase in MIC50, but when $\mathrm{R}_{1}$ and $\mathrm{R}_{2}$ form a cyclohexane (FAI107), there is a 2-fold decrease in the $\mathrm{MIC}_{50}$. To determine if hydrophilic groups alter the activity or potency, we generated analogs with short chains that ended in alcohol groups. Replacing one of the $\mathrm{R}$ groups with an alcohol group resulted in a 2-fold increase in the MIC50 (FAI109, FAI110) while replacing both of the R groups with an alcohol group resulted in an 8-fold increase in the MIC50 (FAI108). The limited loss of potency would indicate that the $\mathrm{R}$ groups have only a modest effect on the ability to interact with Ole1p or potency of the molecule, except for hydrophilic groups that reduce the scaffold's ability to inhibit fungal growth but do not affect target selectivity.

\section{Discussion}

While FA biosynthesis has been successfully targeted to develop antibacterial agents, there have been few fruitful efforts to develop antifungal compounds for this pathway. Drugs like triclosan and isoniazid selectively inhibit bacterial growth by exploiting the fundamental differences between eukaryotic and bacterial FAS, as most prokaryotes use a type II FAS while eukaryotes typically use a type I FAS [86, 87, 163, 182]. Type II FAS systems use separate peptides for each enzyme, while type I FAS 
combines the enzymatic functions into a single complex formed by two peptides [164, 166].

There have been previous reports in C. albicans, Cryptococcus neoformans, and Aspergillus fumigatus of inhibitors of the enzymes of the fungal FA pathway, including a nonselective FAS inhibitor, cerulenin [88, 183]. Cerulenin prevents the synthesis of fatty acids by binding irreversibly to the 3-ketoacyl synthase and blocking the reaction between malonyl-CoA and acetyl-CoA. However, cerulenin is also thought to interfere with sterol synthesis by inhibiting HMG-CoA synthetase [184]. Cerulenin is toxic at 6.7 $\mu \mathrm{M}$ for $C$. albicans, and $C$. neoformans, but $0.2 \mathrm{mM}$ for A. fumigatus [185]. A family of small molecules with a core 1,2,4-thiadiazolidine-3-thione were found to inhibit the fungal FA desaturase [88]. Their mechanism of action was confirmed by restoring growth in the presence of the inhibitors by the addition of UFAs, and FA profiling that demonstrated a substantial loss of UFAs in the membrane after treatment with the inhibitors. Further, selective inhibitors of fungal sphingolipid biosynthesis in Cryptococcus neoformans were identified in a large scale chemical screen, but these compounds had a very narrow spectrum of activity [177]. Unfortunately, none of these compounds have been developed into clinically-relevant therapies. However, from the previous efforts, we know that the enzymes are essential and druggable; therefore, the fungal FA pathway is a prime target for the discovery of novel fungal-specific inhibitors.

We identified very few primary hits for the fungal FAS (31 hits in 41,261 compounds, $0.07 \%$ hit rate), and were unable to confirm any of these hits. While this could simply be a product of the chemical libraries selected, we suspect that this is a direct result of the limited-expression as determined by RTqPCR and narrow susceptibility differential to CRL in TAFiS based dose-responses. Unfortunately, the lack of a validated antibody for the fungal FAS prevented the use of Western blots to compare the protein levels directly. This narrow window to identify chemical-target interactions severely limits the pool's ability to detect on-target inhibitors. The narrow expression differential is most likely due to the complex regulation of the FAS, as we were unable to generate FAS strains with low levels of expression, possibly indicating that a high basal level of FAS expression is required for survival. We attempted to replace the native promoter with multiple promoters of different strengths, but only the replacement with one of the highest expression promoters produced viable colonies. In addition, the overexpression strain with 3 copies of $F A S 1$ had a relatively low basal expression of $F A S 1$, and the same expression as the promoter replacement strain with a single copy of FAS1, which supports the notion that the expression of the FAS complex is heavily regulated at transcription, or in mRNA stability, since the mRNA levels were almost unchanged. Indeed, there are reports in Saccharomyces cerevisiae that document regulatory elements within the ORF of FAS2 [180], and cell cycle-dependent regulation for the entire pathway by an upstream open reading frame that alters translational efficiency but not the transcription [186]. With this extensive regulation, additional techniques and strategies will need to be applied to increase the expression differential of FAS1 if this method is to be applied to identify compounds that interact with this target. It is also possible that more diverse chemical libraries would have yielded more screening hits, and therefore better chances to identify novel inhibitors of the FAS complex. 
However we screened 2 distinct libraries that were specifically designed for diversity, totaling 11,593 compounds with a hit rate of only $0.04 \%$.

We identified 163 primary hits $(0.4 \%$ hit rate) for the Ole $1 \mathrm{p}$ and were able to confirm that 16 of the $59(27 \%)$ tested induced the expected population shift in dose responses. Interestingly, 11 of the $16(69 \%)$ had a hydrazide core that was not shared with the previously identified inhibitor ECC190. The hydrazide containing hits are the most potent compounds of the 16 hits, potentially indicating that the hydrazide core is a promising candidate for further development. We also found that each of these 11 similar compounds, and ECC190, has $\mathrm{pH}$ and medium-dependent inhibition of fungal growth, which will need to be considered in future development. Furthermore, while most of the 16 compounds that induced population shifts were not potent enough to inhibit growth, the addition of UFA was able to restore fungal growth in the presence of the most potent of the primary hits, indicating that the compounds exert growth inhibition through interactions with the desaturase. We attempted to biochemically confirm the Ole $1 p$ hits using fatty acid profiling as Xu and colleagues did when they identified ECC190 [88]. However, the technique provided inconsistent results, for us, even on untreated samples from the same culture.

We identified a series of hydrazide-containing compounds that interact with the fungal FA desaturase. However, only a few of these compounds were potent enough to inhibit the growth of the wild-type strain at the concentrations tested. Therefore we explored the structure-activity relationship of the two most potent hydrazide compounds. The addition or removal of the functional groups on the aromatic ring appears to contribute very little to the potency or the on-target activity of the two compounds. However, any alteration to the central hydrazide core completely eliminated the compound's ability to induce a population shift in the expression pool, indicating that the central hydrazide is essential for these compounds to function as fungal FA desaturase inhibitors. Hydrazide containing compounds have been reported to have high reactivity and toxicity [187, 188] and this limitation will have to be addressed in the future development of these compounds. Furthermore, we were unable to increase the potency of the scaffold, which will need to be considered for future development. Similarly, the toxicity to mammalian cells for these compounds is unknown and will need to be determined before future development.

In addition, we developed analogs to a previously identified Ole1p inhibitor, ECC190, and the degradation product, FAI101. We confirmed that the 1,2,4 triazolidine3-thione broke down into the acylhydrazone, which is similar to a compound previously reported on by Mor and colleagues [170] and Carradori and colleagues [90]. Mor and colleagues identified 2 compounds that are hydrazone/hydrazide combinations with aromatic rings on both sides, termed "hydrazycins," which they postulated that these compounds inhibit fungal sphingolipid synthesis in C. neoformans [170], however, these compounds had poor activity against $C$. albicans. Carradori et al. synthesized (thiazole-2yl)hydrazine derivatives, very similar to FAI101, in order to improve their antifungal spectrum and drug-like properties of a previously identified scaffold [189]. They reported that these had anti-Candida activity similar or better than the antifungal clotrimazole, and 
had limited cytotoxicity on Hep2 cells, a human laryngeal epidermoid carcinoma of the larynx. However, the molecular target or mechanism of these anti-candida agents was never investigated, which we suggest to be the fungal FA desaturase.

Our own SAR efforts discovered that most alterations we made did not alter the molecular target of either ECC190 or FAI101. The exceptions to this observation were the nitro/carboxyl group on the phenyl ring, and the loss of the aromatic nature of the ring. In contrast, the potency of the molecule was more responsive to alterations with the hydrophobic R groups of the molecule contributing very little to the overall potency of the molecule, but hydrophilic additions eliminated potency without altering target engagement. The presence and identity of the thiazole linker was found to be important for the potency. Similarly, substitutions on the phenyl ring decreased the compound's potency, except for the para-substituted halogens. To conclude, the R groups of the compound are flexible for both the activity and the potency, while the phenyl ring has a larger impact on the potency. Collectively, these 2 SAR studies suggest that the TAFiS methodology could prove useful to ensure that analogs developed in SAR retain the original molecular target within whole-cells.

Both FAS and Ole1p had been previously validated as antifungal drug targets and both have established chemical probes, suggesting that they are druggable targets [88, 185]. We identified a novel scaffold that inhibits the growth of $C$. albcians by interacting with Ole1p that is structurally distinct from the previously identified ECC190. The novel scaffold supports the conclusion that Ole1p is a valid and druggable antifungal target. In addition, the identification of the novel scaffold demonstrates that TAFiS can provide an efficient methodology to perform chemical screens that identify inhibitors of traditionally challenging drug targets. In the application to other targets, several target-specific factors will need to be considered. Maximizing the target expression between the strains of the expression pool should increase the range over which on-target inhibitors can be detected. The maximization of expression differential can be challenging with targets like FAS, as multiple forms of regulation directly oppose the stratification of expression. Heavily regulated targets will require additional methods of target stratification, such as silencing mRNA, destabilization of the mRNA (DAmP), activation/deactivation mutations in the target enzyme, and other techniques to alter the sensitivity to on-target inhibitors. Another factor is the secondary effects of altering the target expression, such as membrane permeability or sensitivity to other stresses. Logically, alterations in the structure of the plasma membrane through alterations in the sterol biosynthesis or fatty acid biosynthesis pathways could alter membrane permeability. This concern did not appear to influence our results with FAS, Ole1p, or Erg11p but should still be considered. 


\section{CHAPTER 5. CONCLUSION}

\section{Introduction}

IFIs are estimated to kill over a million people worldwide each year [1] even with our current antifungal treatments. In addition, resistance to existing antifungal therapies is increasing [42], and all classes have high rates of clinical treatment failures [44-48, 54, $55,60,61]$. Therefore, new antifungal therapies are urgently required with improved clinical efficacy. One of the major challenges to discovering novel antifungals is the inefficiency of traditional chemical screening methods [63]. Traditional approaches to drug discovery often yield compounds that lack the characteristics of effective therapies, such as membrane permeability or the ability to engage the molecular target in its native context, i.e. within whole-cells [64], and substantial efforts are required to improve these compounds, extending the timeline and cost of drug discovery. Therefore, the objective of this work was to improve the efficiency of the early stages of drug discovery by establishing and validating the TAFiS methodology, a second-generation target-based whole-cell screening strategy based on the principles of chemical genetics and competitive fitness. TAFiS was specifically designed to increase the efficiency and sensitivity of chemical screening and lead compound selection. By utilizing Dfr1p, which we validated as a promising antifungal target in this work, we demonstrate that our approach reliably and efficiently detects compounds that functionally interact with a defined molecular target in whole cells. We were also able to show that our approach, in applying the method to fungal fatty acid desaturase, can identify novel and physiologically-active inhibitors of proteins that are incompatible with traditional high throughput chemical screening approaches.

\section{Chapter 2: Dihydrofolate Reductase Is a Valid Target for Antifungal Development in the Human Pathogen Candida albicans}

The folate pathway has been exploited to develop both antibacterial and antineoplastic agents, but has received very little attention for antifungal development. While there have been a few attempts to make antifolates with antifungal activity [129, 156], none of these efforts have yielded a viable therapeutic. Indeed, no previous effort had unequivocally demonstrated that DHFR is essential for fungal survival in vitro or in vivo. To test this, we generated a strain of $C$. albicans in which the transcription of $D F R I$ was doxycycline repressible $\left(P_{\text {TETO-DFR }}\right)$ and determined if it was required for growth in vitro and pathogenicity in the mouse model of disseminated candidiasis. Our results confirmed that DHFR is indeed required for $C$. albicans growth in vitro. More importantly, the suppression of DFRI expression in vivo was sufficient to prevent mortality in mice infected with $P_{T E T O}-D F R 1$. To our knowledge, this is the first report that Dfr1p is essential for pathogenicity in vivo, demonstrating that it is a potentially efficacious target for antifungal development. 
In addition, we observed that $C$. albicans has a limited ability to take up or utilize exogenous folates. Only folinic acid was able to partially rescue growth inhibition caused

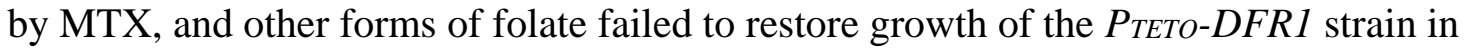
the presence of DOX, indicating that $C$. albicans cannot acquire or utilize sufficient quantities of folate to support growth in the absence of Dfrlp activity. Collectively, our results suggest that $C$. albicans can not acquire sufficient folate derivatives from the mammalian host to survive in the absence of Dfrlp activity. Mammalian cells have a specialized folate transport system that takes up DHF, which is then converted to the active THF by DHFR [136]; in contrast, the majority of prokaryotes and microbial eukaryotes lack folate transporters and must synthesize folic acid de novo. Therefore, it is unlikely that such a transport system exists in $C$. albicans as the uptake of folates would be expected to restore growth in the presence of antifolates and repression of $D F R I$ expression.

While DHFR has been successfully exploited for many years in antibacterial and antineoplastic treatments, we have established it as a valid target for antifungal drug development. The validation of DHFR as a target for antifungal development allows the leveraging of previous efforts that extensively characterized both DHFR and antifolate compounds to devise fungal-selective inhibitors of the folate biosynthetic pathway. Since humans acquire folate from their diet, and microbes synthesize it de novo, several enzymes of the folate biosynthetic pathway are absent in mammals, and may provide an opportunity to develop fungal selective inhibitors of the pathway. DHFR is one of the few proteins in the pathway that is present in humans, however, the relatively wellconserved structure of the fungal and human enzymes likely to pose a significant challenge in developing fungal selective inhibitors and viable therapeutics. While overall human and fungal DHFR is well conserved, there is a basis for selective inhibition as multiple active site residues are not conserved, and the active site is $1.4 \AA$ larger in the fungal DHFR than the human [121]. Indeed, previous reports have shown that trimethoprim analogs, specifically extended to enhance the interactions with the fungal active site, inhibited purified fungal DHFR in the nanomolar range but purified human DHFR in the micromolar range, however toxicity to mammalian cells remained a concern [129]. Therefore, it is probable that the folate pathway proteins without mammalian orthologs are more promising targets with respect to selectivity as small molecules that inhibit these enzymes are less likely to interfere with mammalian folate metabolism and therefore cause less toxicity in humans. Of note, Fol1p and Fol3p are completely absent in humans and poorly characterized in pathogenic fungi, but assuming they are also required for fungal pathogenicity in vivo would make ideal antifungal targets. Neither of these proteins has yet been unequivocally demonstrated to be essential for pathogenic fungi growth or pathogenicity in any model, but Fol1p, a trifunctional enzyme that makes up the majority of the pathway, contains the active site dihydropteroate synthase, a protein already targeted by sulfa antibiotics. 


\section{Chapter 3: Target Abundance-Based Fitness Screening (TAFiS) Facilitates Rapid Identification of Target-Specific and Physiologically Active Chemical Probes}

The TAFiS methodology was designed to address the major failures of traditional chemical screening used at the early stages of drug discovery. Conventional approached yield many hits lacking target-specificity and/or membrane permeability and should increase the efficiency with which promising lead compounds with potential for development into viable therapeutics are identified. In our method, strains with altered expression of the target protein are constructed and labeled with a spectrally distinct FP, and then mixed together to form the expression pool that is used to identify compounds that differentially affect the growth of the strains. The resulting population shifts, detected spectroscopically, indicate that the compound's activity is dependent upon the expression of the target.We developed spectroscopically distinct fluorescent protein constructs that had sufficient signal intensity to allow the quantification of an individual strain's growth within a mixed population. As expected, stratification of the expression of DFRI produces strains with altered susceptibility to the on-target inhibitor, MTX. These strains were mixed together into two separate expression pools and incubated in the presence of MTX, which induced a population shift in both expression pools, indicating that they accurately identify on-target inhibitors. Additionally, incubating the DFRI expression pools with off-target antifungals did not induce population shifts, further confirming the specificity of the assay. By combining the strains with altered target expression together, we achieved higher sensitivity and throughput over screening separate single strains against chemical libraries to identify chemical-target interactions as a result of competitive fitness accentuating chemically-induced fitness defects in the low expression strains.

TAFiS relies on the ability to construct strains with altered susceptibility to compounds that interact with the selected protein and to detect population shifts in mixed cultures using fluorescent protein tags. Heavily regulated targets, especially those that have significant post-transcriptional or post-translational regulation, may have confounding mechanisms that oppose efforts to stratify target expression into the high, medium, and low strains. To use TAFiS against these targets, techniques that altered the stability of the mRNA[150] or abundance of the protein [190,191] would be more useful than the transcriptional stratification we utilized. This is probably why the FAS expression pool had such a limited expression differential, as both subunits are known to under many forms of regulation at both transcriptional and translational levels [186]. Similarly, target proteins that do not have established chemical probes hinder the validation of the TAFiS assay for that target. Without a known interactor, the native susceptibility of the constructed strains remains unknown, and thus a pool of strains with altered susceptibility is challenging as there is no confirmation that target interaction causes the expected population shift. Likewise, compounds or phenotypes that interfere with the detection of the fluorescent signal will also hinder the assay's ability to detect chemical-target interactions. Compounds that fluoresce in the wavelengths used for the FPs or quench the fluorescence of the FPs may yield false positives or negatives. We do not expect this to be a major problem as we identified very few compounds in our screening that were confirmed to interfere with fluorescence detection, however it is 
possible that the reliance on the dTOM FP in the first DFRI pool was partially responsible for the large number of hit compounds that induced negative Z-scores, which were discarded as false positives when they failed to induce the population shift in doseresponse TAFiS assays. We expect many of the fluorescent challenges of TAFiS to be met with advances in the fluorescent protein engineering field, with the production of brighter and more spectrally distinct proteins that are less likely to have compound fluorescent interference.

The TAFiS assay reliably identified inhibitors in both 96- and 384-well plate format. In theory, TAFiS could also be applied in higher throughput screening formats to increase throughput, efficiency, and reduce cost further. However, in the transition from 96-well plates to 384-well plates, the fluorescent signal was reduced by $60 \%$ on average. Therefore the transition to 1536-well plates will have to address the reduction in fluorescent signal, either through more sensitive detection methods or brighter fluorescent proteins. Similarly, the plate reader takes approximately 17 minutes to quantify all four fluorescent wavelengths and the optical density of a single 384-well plate. Therefore, our largest library of 10,000 compounds with 32 screening plates takes over 9 hours to process the entire library. The use of non-commercial automation has reduced the personnel hours required, but increased the amount of time each plate is incubated at room temperature before each signal is quantified, which is suboptimal for the consistency of the assay. Therefore, to increase the throughput of the TAFiS assay, a faster method to quantify each signal in each well would have to be addressed.

Further, we demonstrated that when applied as a primary chemical screen, TAFiS rapidly and specifically identifies compounds known to interact with the selected molecular target in whole cells. This methodology can potentially be applied to nearly any protein in any genetically tractable microbe. TAFiS's ability to rapidly identify compounds that functionally interact with the molecular target, and the elimination of compounds that lack target-specificity or membrane permeability should decrease the cost and time required to obtain promising lead compounds for further development. Having demonstrated the TAFiS methodology using a fungal protein with a highly favorable profile for drug discovery, i.e. a small, globular, soluble protein with a well defined active site, we next sought to determine if the method could provide a viable approach to developing HTP screening assays for a less well characterized target protein that was poorly suited to traditional approaches.

\section{Chapter 4: Identification of Inhibitors of Fungal Fatty Acid Biosynthesis}

One of the biggest advantages of using TAFiS and other TBWCS methodologies is their potential application to traditionally challenging drug targets, such as those that are membrane-bound, to large to purify, or not amenable to biochemical HTP assays. TAFiS was applied to FAS and Ole1p, both of which are previously validated antifungal targets in the fungal fatty acid biosynthetic pathway and are not well suited for traditional approaches to chemical screens as either too large to purify or integrated into membranes, respectively. Both expression pools reliably and reproducibly identified the respective 
inhibitor in TAFiS assay dose-responses, and Z-factors were sufficient for HTP screening applications. Subsequently, we screened a total of 41,261 compounds with each expression pool to identify novel inhibitors of these two targets.

Primary hits were identified for both targets, but only hits from the OLE1 screen had confirmed activity in subsequent dose-response TAFiS assays. The lack of confirmed hits for FAS is likely due to the limited-expression differential between the strains we constructed, as determined by RTqPCR, and susceptibility differential, determined by dose-responses to CRL a known FAS inhibitor, of the FAS expression pool. However, the Ole1p screening identified a family of small molecules with a novel hydrazine core scaffold. Therefore, we have demonstrated that TAFiS can rapidly identify compounds that interact with traditionally challenging targets, such as Ole1p. TAFiS's ability to identify novel inhibitors of targets that were not suited to conventional approaches should increase the range of proteins that are subject to HTP screening for antifungal discovery.

Next, we determined if the TAFiS assay could be used to explore structureactivity relationships (SAR), and to ensure that analogs retained the ability to interact with the original molecular target. Therefore, we chose to identify the structural elements of ECC190, a 1,2,4 triazolidine scaffold previously identified to inhibit Ole1p, and the two most potent hits from the Ole1p screens, both of which contained the hydrazine core. We observed that ECC190 had the potential to degrade into 4-phenyl-2-[2-(propan-2ylidene)hydrazinyl]-1,3-thiazole that was nearly identical to a series of potent antifungal compounds with an unknown molecular target reported by Carradori et al. [90]. An ECC190 analog was synthesized, and the degradation was confirmed by NMR. We further identified structural elements of the breakdown-product that are essential for ECC190 activity against the desaturase using dose-response TAFiS assays to confirm that the analogs inhibited Ole1p. We showed that modification of the thiazole linker, nearly all substitutions to the aromatic ring, or extensive modifications to the $\mathrm{R}$ groups decreased the potency, without affecting the scaffold's ability to inhibit the desaturase. In addition, hydrophilic additions to the $\mathrm{R}$ groups and the loss of aromaticity in the ring eliminated the scaffold's ability to induce a population shift in the Ole1p expression pool, and therefore inhibit the desaturase. This suggests that the inhibitor requires a hydrophobic nature, possibly binding in a hydrophobic pocket and that the aromatic ring probably interacts with other aromatic structures in this binding pocket. In addition, we generated analogs of the hydrazine scaffold we identified represented by FAI354 and FAI409. Each analog was tested for antifungal against a wild-type $C$. albicans strains and against the Ole1p TAFiS assay to ensure that analogs inhibited Ole1p. We found that the central hydrazine core was essential for the hydrazine-based scaffold to induce the population shift in the Ole1p expression pool, and therefore inhibit the enzyme.

Furthermore, we found that substitutions on the aromatic ring had small reductions in the potency of the scaffold. Both the 1,2,4 thiazolidine and hydrazine scaffold SAR studies demonstrate that TAFiS can be used as a tool to ensure that the analogs retain their ontarget activity against the original molecular target. 


\section{Conclusions}

Overall, we have validated a novel and highly sensitive target-based whole-cell screen to detect interactions between small molecules and a specific target protein within whole cells. This approach rapidly eliminates compounds that lack the ability to cross the plasma membrane or target specificity and can be applied to traditionally challenging targets such as Ole1p, a fungal FA desaturase. Furthermore, we have demonstrated that TAFiS can be used as a tool in SAR studies to ensure that the analogs retain the ability to interact with the original molecular target, while still impacting the growth of the whole cell. Due to the potential for TAFiS to be applied to any target in any genetically tractable microbe, we fully expect the development of similar methodologies in other pathogenic fungal species and bacterial species for the identification of promising lead antifungal or antibacterial compounds. Due to the ease of recombinant protein expression, TAFiS could be utilized in the model organism Saccharomyces cerevisiae to express targets from nonculturable microbes, or even disease-relevant human proteins for chemical screening. This novel methodology for chemical screening should decrease the inefficiencies and timeline to discover promising lead compounds. 


\section{LIST OF REFERENCES}

1. Brown, G.D., et al., Hidden killers: human fungal infections. Sci Transl Med, 2012. 4(165): p. 165 rv13.

2. Achkar, J.M. and B.C. Fries, Candida infections of the genitourinary tract. Clinical microbiology reviews, 2010. 23(2): p. 253-273.

3. Garber, G., An overview of fungal infections. Drugs, 2001. 61 Suppl 1: p. 1-12.

4. Gonçalves, B., et al., Vulvovaginal candidiasis: Epidemiology, microbiology and risk factors. Critical reviews in microbiology, 2016. 42(6): p. 905-927.

5. Lamoth, F., et al., Changes in the epidemiological landscape of invasive candidiasis. Journal of Antimicrobial Chemotherapy, 2018. 73(suppl_1): p. i4i13.

6. Wisplinghoff, H., et al., Nosocomial bloodstream infections in US hospitals: analysis of 24,179 cases from a prospective nationwide surveillance study. Clinical infectious diseases : an official publication of the Infectious Diseases Society of America, 2004. 39(3): p. 309-317.

7. Kett, D.H., et al., Candida bloodstream infections in intensive care units: analysis of the extended prevalence of infection in intensive care unit study. Critical care medicine, 2011. 39(4): p. 665-670.

8. Whaley, S.G., et al., Azole Antifungal Resistance in Candida albicans and Emerging Non-albicans Candida Species. Frontiers in Microbiology, 2017. 7: p. 2173.

9. Prevention, C.f.D.C.a. Invasive Candidiasis Statistics. [Web] 2019 [cited 2020 Jan 17th]; Available from: https://www.cdc.gov/fungal/diseases/candidiasis/invasive/statistics.html.

10. Perlroth, J., B. Choi, and B. Spellberg, Nosocomial fungal infections: epidemiology, diagnosis, and treatment. Med Mycol, 2007. 45(4): p. 321-46.

11. Lockhart, S.R., Current epidemiology of Candida infection. Clinical Microbiology Newsletter, 2014. 36(17): p. 131-136.

12. Maertens, J., M. Vrebos, and M. Boogaerts, Assessing risk factors for systemic fungal infections. Eur J Cancer Care (Engl), 2001. 10(1): p. 56-62.

13. Sobel, J.D., Vulvovaginal candidosis. Lancet, 2007. 369(9577): p. 1961-71.

14. Vincent, J.L., et al., Epidemiology, diagnosis and treatment of systemic Candida infection in surgical patients under intensive care. Intensive Care Med, 1998. 24(3): p. 206-16.

15. Wey, S.B., et al., Risk factors for hospital-acquired candidemia. A matched casecontrol study. Arch Intern Med, 1989. 149(10): p. 2349-53.

16. Pfaller, M.A. and D.J. Diekema, Epidemiology of invasive mycoses in North America. Crit Rev Microbiol. 36(1): p. 1-53.

17. Dadar, M., et al., Candida albicans - Biology, molecular characterization, pathogenicity, and advances in diagnosis and control - An update. Microb Pathog, 2018. 117: p. 128-138. 
18. Almirante, B., et al., Epidemiology and Predictors of Mortality in Cases of \&lt;em\&gt;Candida\&lt;/em\&gt; Bloodstream Infection: Results from PopulationBased Surveillance, Barcelona, Spain, from 2002 to 2003. Journal of Clinical Microbiology, 2005. 43(4): p. 1829.

19. Pfaller, M.A., et al., Epidemiology and outcomes of invasive candidiasis due to non-albicans species of Candida in 2,496 patients: data from the Prospective Antifungal Therapy (PATH) registry 2004-2008. PloS one, 2014. 9(7): p. e101510-e101510.

20. Trick, W.E., et al., Secular trend of hospital-acquired candidemia among intensive care unit patients in the United States during 1989-1999. Clinical infectious diseases : an official publication of the Infectious Diseases Society of America, 2002. 35(5): p. 627-630.

21. Kaushik, N., G.G. Pujalte, and S.T. Reese, Superficial Fungal Infections. Prim Care, 2015. 42(4): p. 501-16.

22. Gaffen, S.L. and N.M. Moutsopoulos, Regulation of host-microbe interactions at oral mucosal barriers by type 17 immunity. Sci Immunol, 2020. 5(43).

23. Delsing, C.E., et al., Association of esophageal candidiasis and squamous cell carcinoma. Med Mycol Case Rep, 2012. 1(1): p. 5-8.

24. Zhu, F., et al., Autoreactive T Cells and Chronic Fungal Infection Drive Esophageal Carcinogenesis. Cell Host Microbe, 2017. 21(4): p. 478-493.e7.

25. Nyirjesy, P., et al., Causes of chronic vaginitis: analysis of a prospective database of affected women. Obstet Gynecol, 2006. 108(5): p. 1185-91.

26. Telles, D.R., N. Karki, and M.W. Marshall, Oral fungal infections: diagnosis and management. Dental Clinics, 2017. 61(2): p. 319-349.

27. Neville, B.A., C. d'Enfert, and M.-E. Bougnoux, Candida albicans commensalism in the gastrointestinal tract. FEMS Yeast Research, 2015. 15(7).

28. Nobile, C.J. and A.D. Johnson, Candida albicans Biofilms and Human Disease. Annual review of microbiology, 2015. 69: p. 71-92.

29. Mayer, F.L., D. Wilson, and B. Hube, Candida albicans pathogenicity mechanisms. Virulence, 2013. 4(2): p. 119-128.

30. Moyes, D.L., et al., Candidalysin is a fungal peptide toxin critical for mucosal infection. Nature, 2016. 532(7597): p. 64-68.

31. Vermitsky, J.P. and T.D. Edlind, Azole resistance in Candida glabrata: coordinate upregulation of multidrug transporters and evidence for a Pdrl-like transcription factor. Antimicrob Agents Chemother, 2004. 48(10): p. 3773-81.

32. McMullan, R., et al., Trends in the epidemiology of Candida bloodstream infections in Northern Ireland between January 1984 and December 2000. J Infect, 2002. 45(1): p. 25-8.

33. Pfaller, M.A., et al., Trends in species distribution and susceptibility to fluconazole among blood stream isolates of Candida species in the United States. Diagn Microbiol Infect Dis, 1999. 33(4): p. 217-22.

34. Price, M.F., M.T. LaRocco, and L.O. Gentry, Fluconazole susceptibilities of Candida species and distribution of species recovered from blood cultures over a 5-year period. Antimicrob Agents Chemother, 1994. 38(6): p. 1422-4. 
35. Newman, S.L., et al., Clinically significant mucosal candidiasis resistant to fluconazole treatment in patients with AIDS. Clin Infect Dis, 1994. 19(4): p. 6846.

36. Spivak, E.S. and K.E. Hanson, Candida auris: an Emerging Fungal Pathogen. J Clin Microbiol, 2018. 56(2).

37. Goodwin, S.D., et al., Pretreatment regimens for adverse events related to infusion of amphotericin B. Clin Infect Dis, 1995. 20(4): p. 755-61.

38. Chen, S.C., M.A. Slavin, and T.C. Sorrell, Echinocandin antifungal drugs in fungal infections: a comparison. Drugs. 71(1): p. 11-41.

39. Wiederhold, N.P., The antifungal arsenal: alternative drugs and future targets. International Journal of Antimicrobial Agents, 2018. 51(3): p. 333-339.

40. Robbins, N., G.D. Wright, and L.E. Cowen, Antifungal Drugs: The Current Armamentarium and Development of New Agents. Microbiology spectrum, 2016. 4(5): p. 10.1128/microbiolspec.FUNK-0002-2016.

41. Shapiro, R.S., N. Robbins, and L.E. Cowen, Regulatory circuitry governing fungal development, drug resistance, and disease. Microbiology and molecular biology reviews : MMBR, 2011. 75(2): p. 213-267.

42. Berkow, E.L. and S.R. Lockhart, Fluconazole resistance in Candida species: a current perspective. Infection and Drug Resistance, 2017. 10: p. 237-245.

43. Rex, J.H., et al., Development of interpretive breakpoints for antifungal susceptibility testing: conceptual framework and analysis of in vitro-in vivo correlation data for fluconazole, itraconazole, and candida infections.

Subcommittee on Antifungal Susceptibility Testing of the National Committee for Clinical Laboratory Standards. Clin Infect Dis, 1997. 24(2): p. 235-47.

44. Reboli, A.C., et al., Anidulafungin versus fluconazole for invasive candidiasis. N Engl J Med, 2007. 356(24): p. 2472-82.

45. Rex, J.H., et al., A randomized trial comparing fluconazole with amphotericin $B$ for the treatment of candidemia in patients without neutropenia. Candidemia Study Group and the National Institute. N Engl J Med, 1994. 331(20): p. 1325-30.

46. Kett, D.H., et al., Anidulafungin compared with fluconazole in severely ill patients with candidemia and other forms of invasive candidiasis: support for the 2009 IDSA treatment guidelines for candidiasis. Crit Care, 2011. 15(5): p. R253.

47. Kullberg, B.J., et al., Voriconazole versus a regimen of amphotericin $B$ followed by fluconazole for candidaemia in non-neutropenic patients: a randomised noninferiority trial. Lancet, 2005. 366(9495): p. 1435-42.

48. Kullberg, B.J., et al., Isavuconazole versus Caspofungin in the Treatment of Candidemia and Other Invasive Candida Infections: The ACTIVE Trial. Clin Infect Dis, 2018.

49. Gruszecki, W.I., et al., Organization of antibiotic amphotericin B in model lipid membranes. A mini review. Cellular \& molecular biology letters, 2003. 8(1): p. 161-170.

50. Anderson, T.M., et al., Amphotericin forms an extramembranous and fungicidal sterol sponge. Nature chemical biology, 2014. 10(5): p. 400-406.

51. Hamill, R.J., Amphotericin B Formulations: A Comparative Review of Efficacy and Toxicity. Drugs, 2013. 73(9): p. 919-934. 
52. Ostrosky-Zeichner, L., et al., An insight into the antifungal pipeline: selected new molecules and beyond. Nature Reviews Drug Discovery, 2010. 9(9): p. 719-727.

53. Vincent, B.M., et al., Fitness trade-offs restrict the evolution of resistance to amphotericin B. PLoS biology, 2013. 11(10): p. e1001692-e1001692.

54. Mora-Duarte, J., et al., Comparison of caspofungin and amphotericin B for invasive candidiasis. N Engl J Med, 2002. 347(25): p. 2020-9.

55. Walsh, T.J., et al., Caspofungin versus liposomal amphotericin B for empirical antifungal therapy in patients with persistent fever and neutropenia. $\mathrm{N}$ Engl J Med, 2004. 351(14): p. 1391-402.

56. Butts, A. and D.J. Krysan, Antifungal Drug Discovery: Something Old and Something New. PLOS Pathogens, 2012. 8(9): p. e1002870.

57. Chang, C.C., M.A. Slavin, and S.C.A. Chen, New developments and directions in the clinical application of the echinocandins. Archives of Toxicology, 2017. 91(4): p. 1613-1621.

58. Hector, R.F. and D.E. Bierer, New $\beta$-glucan inhibitors as antifungal drugs. Expert Opinion on Therapeutic Patents, 2011. 21(10): p. 1597-1610.

59. Denning, D.W., Echinocandin antifungal drugs. Lancet (London, England), 2003. 362(9390): p. 1142-1151.

60. Betts, R.F., et al., A Multicenter, double-blind trial of a high-dose caspofungin treatment regimen versus a standard caspofungin treatment regimen for adult patients with invasive candidiasis. Clin Infect Dis, 2009. 48(12): p. 1676-84.

61. Pappas, P.G., et al., Micafungin versus caspofungin for treatment of candidemia and other forms of invasive candidiasis. Clin Infect Dis, 2007. 45(7): p. 883-93.

62. DiMasi, J.A., H.G. Grabowski, and R.W. Hansen, Innovation in the pharmaceutical industry: New estimates of $R \& D$ costs. J Health Econ, 2016. 47: p. 20-33.

63. Moffat, J.G., J. Rudolph, and D. Bailey, Phenotypic screening in cancer drug discovery - past, present and future. Nat Rev Drug Discov, 2014. 13(8): p. 588602.

64. Pucci, M.J., T.J. Dougherty, and J.F. Barrett, Why are there no new antibiotics? Expert Opinion on Investigational Drugs, 1998. 7(8): p. 1233-1235.

65. Schenone, M., et al., Target identification and mechanism of action in chemical biology and drug discovery. Nat Chem Biol, 2013. 9(4): p. 232-40.

66. Chidley, C., et al., Searching for the protein targets of bioactive molecules. Chimia (Aarau), 2011. 65(9): p. 720-4.

67. Karathia, H., et al., Saccharomyces cerevisiae as a model organism: a comparative study. PloS one, 2011. 6(2): p. e16015-e16015.

68. Harrison, R.K., Phase II and phase III failures: 2013-2015. 2016, Nature Publishing Group.

69. Arrowsmith, J., Trial watch: phase III and submission failures: 2007-2010. 2011, Nature Publishing Group.

70. Stockwell, B.R., Chemical genetics: ligand-based discovery of gene function. Nature reviews. Genetics, 2000. 1(2): p. 116-125.

71. Singh, S.B., J.W. Phillips, and J. Wang, Highly sensitive target-based whole-cell antibacterial discovery strategy by antisense RNA silencing. Curr Opin Drug Discov Devel, 2007. 10(2): p. 160-6. 
72. Miller, C.H., et al., Modifying culture conditions in chemical library screening identifies alternative inhibitors of mycobacteria. Antimicrob Agents Chemother, 2009. 53(12): p. 5279-83.

73. Bonnett, S.A., et al., A Target-Based Whole Cell Screen Approach To Identify Potential Inhibitors of Mycobacterium tuberculosis Signal Peptidase. ACS Infectious Diseases, 2016. 2(12): p. 893-902.

74. Young, K., et al., Discovery of FabH/FabF inhibitors from natural products. Antimicrobial agents and chemotherapy, 2006. 50(2): p. 519-526.

75. Neradil, J., G. Pavlasova, and R. Veselska, New mechanisms for an old drug; DHFR- and non-DHFR-mediated effects of methotrexate in cancer cells. Klinicka onkologie : casopis Ceske a Slovenske onkologicke spolecnosti, 2012. 25 Suppl 2: p. 2S87-92.

76. Sramek, M., J. Neradil, and R. Veselska, Much more than you expected: The nonDHFR-mediated effects of methotrexate. Biochimica et Biophysica Acta (BBA) General Subjects, 2017. 1861(3): p. 499-503.

77. Gleckman, R., N. Blagg, and D.W. Joubert, Trimethoprim: mechanisms of action, antimicrobial activity, bacterial resistance, pharmacokinetics, adverse reactions, and therapeutic indications. Pharmacotherapy, 1981. 1(1): p. 14-20.

78. Darlow, B., et al., Sulfadoxine-pyrimethamine for the treatment of acute malaria in children in Papua New Guinea. I. Plasmodium falciparum. The American journal of tropical medicine and hygiene, 1982. 31(1): p. 1-9.

79. Meltzer, E., G. Rahav, and E. Schwartz, Vivax Malaria Chemoprophylaxis: The Role of Atovaquone-Proguanil Compared to Other Options. Clinical infectious diseases : an official publication of the Infectious Diseases Society of America, 2018. 66(11): p. 1751-1755.

80. Bukirwa, H., P. Garner, and J. Critchley, Chlorproguanil-dapsone for treating uncomplicated malaria. The Cochrane database of systematic reviews, 2004. 2004(4): p. CD004387-CD004387.

81. Wolf, R., et al., Dapsone. Dermatology online journal, 2002. 8(1): p. 2-2.

82. Avino, L.J., S.M. Naylor, and A.M. Roecker, Pneumocystis jirovecii Pneumonia in the Non-HIV-Infected Population. The Annals of pharmacotherapy, 2016. 50(8): p. 673-679.

83. Navarro-Martinez, M.D., J. Cabezas-Herrera, and J.N. Rodriguez-Lopez, Antifolates as antimycotics? Connection between the folic acid cycle and the ergosterol biosynthesis pathway in Candida albicans. Int J Antimicrob Agents, 2006. 28(6): p. 560-7.

84. Warnock, D.W., et al., Effect of methotrexate alone and in combination with antifungal drugs on the growth of Candida albicans. J Antimicrob Chemother, 1989. 23(6): p. 837-47.

85. Fekete-Forgacs, K., et al., Effect of fluconazole on the growth and adhesion of Candida albicans in the presence of antineoplastic agents. J Basic Microbiol, 1999. 39(5-6): p. 305-10.

86. Banerjee, A., et al., inhA, a gene encoding a target for isoniazid and ethionamide in Mycobacterium tuberculosis. Science, 1994. 263(5144): p. 227. 
87. Heath, R.J., et al., Broad spectrum antimicrobial biocides target the FabI component of fatty acid synthesis. The Journal of biological chemistry, 1998. 273(46): p. 30316-30320.

88. Xu, D., et al., Chemical Genetic Profiling and Characterization of Smallmolecule Compounds That Affect the Biosynthesis of Unsaturated Fatty Acids in Candida albicans. Journal of Biological Chemistry, 2009. 284(29): p. 1975419764.

89. Carradori, S., et al., Synthesis, biological evaluation and quantitative structureactive relationships of 1,3-thiazolidin-4-one derivatives. A promising chemical scaffold endowed with high antifungal potency and low cytotoxicity. Eur J Med Chem, 2017. 140: p. 274-292.

90. Carradori, S., et al., Synthesis and cytotoxicity of novel (thiazol-2-yl)hydrazine derivatives as promising anti-Candida agents. Eur J Med Chem, 2013. 65: p. 10211 .

91. Benedict, K., et al., Estimation of Direct Healthcare Costs of Fungal Diseases in the United States. Clin Infect Dis, 2019. 68(11): p. 1791-1797.

92. Tiphine, M., V. Letscher-Bru, and R. Herbrecht, Amphotericin B and its new formulations: pharmacologic characteristics, clinical efficacy, and tolerability. Transpl Infect Dis, 1999. 1(4): p. 273-83.

93. Rex, J.H., et al., A Randomized Trial Comparing Fluconazole with Amphotericin $B$ for the Treatment of Candidemia in Patients without Neutropenia. New England Journal of Medicine, 1994. 331(20): p. 1325-1330.

94. Reboli, A.C., et al., Anidulafungin compared with fluconazole for treatment of candidemia and other forms of invasive candidiasis caused by Candida albicans: a multivariate analysis of factors associated with improved outcome. BMC Infect Dis, 2011. 11: p. 261.

95. Liu, J., et al., Towards new antifolates targeting eukaryotic opportunistic infections. Eukaryot Cell, 2009. 8(4): p. 483-6.

96. Hou, Z. and L.H. Matherly, Biology of the major facilitative folate transporters SLC19A1 and SLC46A1. Curr Top Membr, 2014. 73: p. 175-204.

97. DeJarnette C, Luna-Tapia A, Estredge LR, Palmer GE. 2020. Dihydrofolate reductase is a valid target for antifungal development in the human pathogen Candida albicans. mSphere 5:e00374-20. https://doi.org/10.1128/mSphere.00374-20.

98. Burke, D., Methods in yeast genetics : a Cold Spring Harbor Laboratory course manual, D. Dawson and T. Stearns, Editors. 2000, Cold Spring Harbor Laboratory Press: Plainview, N.Y.:

99. Bryce Wilson, R., et al., A recyclable Candida albicans URA3 cassette for PCR product-directed gene disruptions. Yeast, 2000. 16(1): p. 65-70.

100. Gietz, D., et al., Improved method for high efficiency transformation of intact yeast cells. Nucleic Acids Research, 1992. 20(6): p. 1425-1425.

101. Gerami-Nejad, M., et al., Shuttle vectors for facile gap repair cloning and integration into a neutral locus in Candida albicans. Microbiology (Reading, England), 2013. 159(Pt 3): p. 565-579. 
102. Nakayama, H., et al., Tetracycline-regulatable system to tightly control gene expression in the pathogenic fungus Candida albicans. Infection and immunity, 2000. 68(12): p. 6712-6719.

103. Wilson, R.B., D. Davis, and A.P. Mitchell, Rapid Hypothesis Testing with Candida albicans through Gene Disruption with Short Homology Regions. Journal of Bacteriology, 1999. 181(6): p. 1868-1874.

104. Butts, A., et al., Target Abundance-Based Fitness Screening (TAFiS) Facilitates Rapid Identification of Target-Specific and Physiologically Active Chemical Probes. mSphere, 2017. 2(5): p. e00379-17.

105. Livak, K.J. and T.D. Schmittgen, Analysis of Relative Gene Expression Data Using Real-Time Quantitative PCR and the 2- $\triangle \triangle C T$ Method. Methods, 2001. 25(4): p. 402-408.

106. Skrzypek, M.S., et al., The Candida Genome Database (CGD): incorporation of Assembly 22, systematic identifiers and visualization of high throughput sequencing data. Nucleic acids research, 2017. 45(D1): p. D592-D596.

107. Calder, R.B., et al., Cloning and characterization of a eukaryotic pantothenate kinase gene (panK) from Aspergillus nidulans. J Biol Chem, 1999. 274(4): p. 2014-20.

108. Daly, S., et al., Sequence of a dihydrofolate reductase-encoding gene from Candida albicans. Gene, 1994. 147(1): p. 115-118.

109. Baccanari, D.P., et al., Characterization of Candida albicans dihydrofolate reductase. J Biol Chem, 1989. 264(2): p. 1100-7.

110. Little, J.G. and R.H. Haynes, Isolation and characterization of yeast mutants auxotrophic for 2'-deoxythymidine 5'-monophosphate. Mol Gen Genet, 1979. 168(2): p. 141-51.

111. Huang, T., et al., The phenotype of a dihydrofolate reductase mutant of Saccharomyces cerevisiae. Gene, 1992. 121(1): p. 167-171.

112. Güldener, U., et al., Characterization of the Saccharomyces cerevisiae Foll protein: starvation for C1 carrier induces pseudohyphal growth. Molecular biology of the cell, 2004. 15(8): p. 3811-3828.

113. George, L., et al., Plasma Folate Levels and Risk of Spontaneous Abortion. JAMA, 2002. 288(15): p. 1867-1873.

114. de Benoist, B., Conclusions of a WHO Technical Consultation on folate and vitamin B12 deficiencies. Food and nutrition bulletin, 2008. 29(2 Suppl): p. S238S244.

115. Fairbanks VF, K.G., Biochemical Aspects of Hematology, in Tietz Textbook of Clinical Chemistry, C. Burtis, Editor. 1999, WB Saunders Company: Philadelphia. p. 1690-1698.

116. Luna-Tapia, A., A. Butts, and G.E. Palmer, Loss of C-5 Sterol Desaturase Activity in Candida albicans: Azole Resistance or Merely Trailing Growth? Antimicrobial agents and chemotherapy, 2018. 63(1): p. e01337-18.

117. Harry, J.B., et al., Drug-induced regulation of the MDR1 promoter in Candida albicans. Antimicrobial agents and chemotherapy, 2005. 49(7): p. 2785-2792.

118. Ben-Yaacov, R., et al., Candida albicans gene encoding resistance to benomyl and methotrexate is a multidrug resistance gene. Antimicrobial agents and chemotherapy, 1994. 38(4): p. 648-652. 
119. Hochuli, E., et al., Genetic Approach to Facilitate Purification of Recombinant Proteins with a Novel Metal Chelate Adsorbent. Bio/Technology, 1988. 6(11): p. 1321-1325.

120. Young, C.L., Z.T. Britton, and A.S. Robinson, Recombinant protein expression and purification: a comprehensive review of affinity tags and microbial applications. Biotechnology journal, 2012. 7(5): p. 620-634.

121. Whitlow, M., et al., X-ray crystallographic studies of Candida albicans dihydrofolate reductase. High resolution structures of the holoenzyme and an inhibited ternary complex. J Biol Chem, 1997. 272(48): p. 30289-98.

122. Rossi, G., et al., The evolving role of pemetrexed disodium for the treatment of non-small cell lung cancer. Expert Opin Pharmacother, 2018. 19(17): p. 19691976.

123. Marques, S.A., Paracoccidioidomycosis. Clinics in Dermatology, 2012. 30(6): p. 610-615.

124. Otzen, T., et al., Folate-synthesizing enzyme system as target for development of inhibitors and inhibitor combinations against Candida albicans-synthesis and biological activity of new 2,4-diaminopyrimidines and 4'-substituted 4aminodiphenyl sulfones. J Med Chem, 2004. 47(1): p. 240-53.

125. Liu, N., et al., Emerging New Targets for the Treatment of Resistant Fungal Infections. J Med Chem, 2018. 61(13): p. 5484-5511.

126. Hynes, J.B., et al., Activity of selected 2,4-diaminoquinazolines against Candida albicans in vitro. Proc Soc Exp Biol Med, 1976. 153(2): p. 230-2.

127. Chan, J.H., et al., Selective inhibitors of Candida albicans dihydrofolate reductase: activity and selectivity of 5-(arylthio)-2,4-diaminoquinazolines. J Med Chem, 1995. 38(18): p. 3608-16.

128. Liu, J., et al., Structure-guided development of efficacious antifungal agents targeting Candida glabrata dihydrofolate reductase. Chem Biol, 2008. 15(9): p. 990-6.

129. Paulsen, J.L., et al., In vitro biological activity and structural analysis of 2,4diamino-5-(2'-arylpropargyl)pyrimidine inhibitors of Candida albicans. Bioorganic \& medicinal chemistry, 2009. 17(14): p. 4866-4872.

130. Bermingham, A. and J.P. Derrick, The folic acid biosynthesis pathway in bacteria: evaluation of potential for antibacterial drug discovery. Bioessays, 2002. 24(7): p. 637-48.

131. Güldener, U., et al., Characterization of the Saccharomyces cerevisiae Foll protein: starvation for Cl carrier induces pseudohyphal growth. Mol Biol Cell, 2004. 15(8): p. 3811-28.

132. Cherest, H., D. Thomas, and Y. Surdin-Kerjan, Polyglutamylation of folate coenzymes is necessary for methionine biosynthesis and maintenance of intact mitochondrial genome in Saccharomyces cerevisiae. J Biol Chem, 2000. 275(19): p. 14056-63.

133. Segal, E.S., et al., Gene Essentiality Analyzed by In Vivo Transposon Mutagenesis and Machine Learning in a Stable Haploid Isolate of Candida albicans. mBio, 2018. 9(5).

134. Meir, Z. and N. Osherov, Vitamin Biosynthesis as an Antifungal Target. J Fungi (Basel), 2018. 4(2). 
135. Westerhof, G.R., et al., Carrier-and receptor-mediated transport of folate antagonists targeting folate-dependent enzymes: correlates of molecular-structure and biological activity. Molecular pharmacology, 1995. 48(3): p. 459-471.

136. Information, N.C.f.B. Methotrexate $C I D=126941$. [cited 2020; Available from: https://pubchem.ncbi.nlm.nih.gov/compound/Methotrexate.

137. G-Dayanandan, N., et al., Propargyl-Linked Antifolates are Dual Inhibitors of Candida albicans and Candida glabrata. Journal of Medicinal Chemistry, 2014. 57(6): p. 2643-2656.

138. Paulsen, J.L., et al., Structural analysis of the active sites of dihydrofolate reductase from two species of Candida uncovers ligand-induced conformational changes shared among species. Bioorganic \& medicinal chemistry letters, 2013. 23(5): p. 1279-1284.

139. Liu, M., et al., Conserved Fungal Genes as Potential Targets for Broad-Spectrum Antifungal Drug Discovery. Eukaryotic Cell, 2006. 5(4): p. 638-649.

140. Butts A, DeJarnette C, Peters TL, Parker JE, Kerns ME, Eberle KE, Kelly SL, Palmer GE. 2017. Target abundance-based fitness screening (TAFiS) facilitates rapid identification of target-specific and physiologically active chemical probes. mSphere 2:e00379-17. https://doi.org/10.1128/mSphere.00379-17

141. Ramón, A.M. and W.A. Fonzi, Diverged Binding Specificity of Rim101p, the Candida albicans Ortholog of PacC. Eukaryotic Cell, 2003. 2(4): p. 718-728.

142. Gerami-Nejad, M., K. Dulmage, and J. Berman, Additional cassettes for epitope and fluorescent fusion proteins in Candida albicans. Yeast, 2009. 26(7): p. 399406.

143. Gerami-Nejad, M., J. Berman, and C.A. Gale, Cassettes for PCR-mediated construction of green, yellow, and cyan fluorescent protein fusions in Candida albicans. Yeast, 2001. 18(9): p. 859-64.

144. Gratacap, R.L., J.F. Rawls, and R.T. Wheeler, Mucosal candidiasis elicits NFkappaB activation, proinflammatory gene expression and localized neutrophilia in zebrafish. Dis Model Mech, 2013. 6(5): p. 1260-70.

145. Ai, H.W., et al., Exploration of new chromophore structures leads to the identification of improved blue fluorescent proteins. Biochemistry, 2007. 46(20): p. 5904-10.

146. Zhang, C. and J.B. Konopka, A photostable green fluorescent protein variant for analysis of protein localization in Candida albicans. Eukaryot Cell, 2010. 9(1): p. 224-6.

147. Johnston, D.A., et al., Three Prevacuolar Compartment Rab GTPases Impact Candida albicans Hyphal Growth. Eukaryotic Cell, 2013. 12(7): p. 1039-1050.

148. Luna-Tapia, A., et al., ERG2 and ERG24 Are Required for Normal Vacuolar Physiology as Well as Candida albicans Pathogenicity in a Murine Model of Disseminated but Not Vaginal Candidiasis. Eukaryotic Cell, 2015. 14(10): p. 1006-1016.

149. Fonzi, W.A. and M.Y. Irwin, Isogenic strain construction and gene mapping in Candida albicans. Genetics, 1993. 134(3): p. 717-728.

150. Finkel, J.S., et al., Application of the systematic "DAmP" approach to create a partially defective C. albicans mutant. Fungal Genet Biol, 2011. 48(11): p. 105661. 
151. Schmitt, M.E., T.A. Brown, and B.L. Trumpower, A rapid and simple method for preparation of RNA from Saccharomyces cerevisiae. Nucleic Acids Research, 1990. 18(10): p. 3091-3092.

152. Markwardt, M.L., et al., An Improved Cerulean Fluorescent Protein with Enhanced Brightness and Reduced Reversible Photoswitching. PLOS ONE, 2011. 6(3): p. e17896.

153. Shagin, D.A., et al., GFP-like Proteins as Ubiquitous Metazoan Superfamily: Evolution of Functional Features and Structural Complexity. Molecular Biology and Evolution, 2004. 21(5): p. 841-850.

154. Matz, M., Amplification of cDNA ends based on template-switching effect and step-out PCR. Nucleic Acids Res., 1999. 27: p. 1558-1560.

155. Tsien, R.Y. and L. Wang, Red-shifted fluorescent proteins mPlum and mRaspberry and polynucleotides encoding the same. 2008, Google Patents.

156. Paulsen, J.L., S.D. Bendel, and A.C. Anderson, Crystal structures of Candida albicans dihydrofolate reductase bound to propargyl-linked antifolates reveal the flexibility of active site loop residues critical for ligand potency and selectivity. Chem Biol Drug Des, 2011. 78(4): p. 505-12.

157. Bolstad, D.B., et al., Dihydrofolate reductase inhibitors: developments in antiparasitic chemotherapy. Expert Opinion on Therapeutic Patents, 2008. 18(2): p. 143-157.

158. Nzila, A., The past, present and future of antifolates in the treatment of Plasmodium falciparum infection. Journal of Antimicrobial Chemotherapy, 2006. 57(6): p. 1043-1054.

159. Zhang, J.-H., T.D.Y. Chung, and K.R. Oldenburg, A Simple Statistical Parameter for Use in Evaluation and Validation of High Throughput Screening Assays. Journal of Biomolecular Screening, 1999. 4(2): p. 67-73.

160. Shaner, N.C., G.H. Patterson, and M.W. Davidson, Advances in fluorescent protein technology. J Cell Sci, 2007. 120(Pt 24): p. 4247-60.

161. Shaner, N.C., et al., Improved monomeric red, orange and yellow fluorescent proteins derived from Discosoma sp. red fluorescent protein. Nature biotechnology, 2004. 22(12): p. 1567-1572.

162. Patil, A. and S. Majumdar, Echinocandins in antifungal pharmacotherapy. The Journal of pharmacy and pharmacology, 2017. 69(12): p. 1635-1660.

163. Tehlivets, O., K. Scheuringer, and S.D. Kohlwein, Fatty acid synthesis and elongation in yeast. Biochim Biophys Acta, 2007. 1771(3): p. 255-70.

164. Leibundgut, M., et al., The multienzyme architecture of eukaryotic fatty acid synthases. Curr Opin Struct Biol, 2008. 18(6): p. 714-25.

165. Parsons, J.B. and C.O. Rock, Is bacterial fatty acid synthesis a valid target for antibacterial drug discovery? Current opinion in microbiology, 2011. 14(5): p. 544-549.

166. Jenni, S., et al., Architecture of a fungal fatty acid synthase at 5 A resolution. Science, 2006. 311(5765): p. 1263-7.

167. Stoops, J.K., et al., Presence of two polypeptide chains comprising fatty acid synthetase. Proceedings of the National Academy of Sciences of the United States of America, 1975. 72(5): p. 1940-1944. 
168. Krishnamurthy, S., et al., Dosage-dependent functions of fatty acid desaturase Ole1p in growth and morphogenesis of Candida albicans. Microbiology, 2004. 150(Pt 6): p. 1991-2003.

169. Zhao, X.J., et al., Avirulence of Candida albicans FAS2 mutants in a mouse model of systemic candidiasis. Infect Immun, 1997. 65(2): p. 829-32.

170. Lazzarini, C., et al., Acylhydrazones as Antifungal Agents Targeting the Synthesis of Fungal Sphingolipids. Antimicrob Agents Chemother, 2018. 62(5).

171. Nguyen, L.N., D. Trofa, and J.D. Nosanchuk, Fatty acid synthase impacts the pathobiology of Candida parapsilosis in vitro and during mammalian infection. PLoS One, 2009. 4(12): p. e8421.

172. Seddon, A.M., P. Curnow, and P.J. Booth, Membrane proteins, lipids and detergents: not just a soap opera. Biochimica et biophysica acta, 2004. 1666(1-2): p. $105-117$.

173. Lundgren, A., et al., Affinity Purification and Single-Molecule Analysis of Integral Membrane Proteins from Crude Cell-Membrane Preparations. Nano Letters, 2018. 18(1): p. 381-385.

174. Yang, H., et al., Purification of a Large Protein Using Ion-Exchange Membranes. Industrial \& Engineering Chemistry Research, 2002. 41(6): p. 1597-1602.

175. Sakakibara, H. and K. Oiwa, Molecular organization and force-generating mechanism of dynein. The FEBS Journal, 2011. 278(17): p. 2964-2979.

176. Xu, D., et al., Genome-wide fitness test and mechanism-of-action studies of inhibitory compounds in Candida albicans. PLoS Pathog, 2007. 3(6): p. e92.

177. Mor, V., et al., Identification of a New Class of Antifungals Targeting the Synthesis of Fungal Sphingolipids. mBio, 2015. 6(3): p. e00647-e00647.

178. Burke, D., D. Dawson, and T. Stearns, Methods in yeast genetics: a Cold Spring Harbor Laboratory course manual. 2000: CSHL Press.

179. Gaulton, A., et al., The ChEMBL database in 2017. Nucleic Acids Research, 2017. 45(Database issue): p. D945-D954.

180. Wenz, P., et al., A downstream regulatory element located within the coding sequence mediates autoregulated expression of the yeast fatty acid synthase gene FAS2 by the FAS1 gene product. Nucleic Acids Research, 2001. 29(22): p. 46254632.

181. Wayne, P., Method for antifungal disk diffusion susceptibility testing of yeasts. CLSI m44-a, 2004.

182. Lu, H. and P.J. Tonge, Inhibitors of FabI, an enzyme drug target in the bacterial fatty acid biosynthesis pathway. Acc Chem Res, 2008. 41(1): p. 11-20.

183. Yan, C., et al., The mTOR inhibitor rapamycin synergizes with a fatty acid synthase inhibitor to induce cytotoxicity in ER/HER2-positive breast cancer cells. PloS one, 2014. 9(5): p. e97697-e97697.

184. Ohno, T., et al., Mechanism of action of CM-55, a synthetic analogue of the antilipogenic antibiotic cerulenin. Antimicrob Agents Chemother, 1974. 6(4): p. 387-92.

185. Omura, S., The antibiotic cerulenin, a novel tool for biochemistry as an inhibitor of fatty acid synthesis. Bacteriological reviews, 1976. 40(3): p. 681.

186. Blank, H.M., et al., Translational control of lipogenic enzymes in the cell cycle of synchronous, growing yeast cells. Embo j, 2017. 36(4): p. 487-502. 
187. Venkatachalam, K., et al., Biochemical and molecular aspects of 1,2dimethylhydrazine (DMH)-induced colon carcinogenesis: a review. Toxicol Res (Camb), 2020. 9(1): p. 2-18.

188. Penketh, P., H. Williamson, and K. Shyam, Physicochemical considerations of tumor selective drug delivery and activity confinement with particular reference to 1, 2-bis (sulfonyl)-1-alkylhydrazines delivery. Current Drug Delivery, 2020.

189. Chimenti, F., et al., Synthesis and in vitro activity of 2-thiazolylhydrazone derivatives compared with the activity of clotrimazole against clinical isolates of Candida spp. Bioorg Med Chem Lett, 2007. 17(16): p. 4635-40.

190. Varshavsky, A. The $N$-end rule. in Cold Spring Harbor symposia on quantitative biology. 1995. Cold Spring Harbor Laboratory Press.

191. Natsume, T. and M.T. Kanemaki, Conditional degrons for controlling protein expression at the protein level. Annual Review of Genetics, 2017. 51: p. 83-102. 


\section{VITA}

Christian Alexander DeJarnette, son of Ken and Tammi DeJarnette, was born in Suffolk, VA, in 1993. He attended and graduated from Martin Luther King Jr. Academic Magnet for Health Sciences and Engineering in Nashville, TN, in 2011. Then he pursued and obtained a Bachelor of Science in Biochemistry from Lee University in Cleveland, $\mathrm{TN}$, in 2015. Then Christian started the Molecular Biology, Immunology, and Biochemistry program at the University of Tennessee Health Science Center and is expected to complete the requirements for the doctoral degree by October 2020. 